\title{
THE WEIL PROOF AND THE GEOMETRY OF THE ADELES CLASS SPACE
}

\author{
ALAIN CONNES, CATERINA CONSANI, AND MATILDE MARCOLLI \\ Dedicated to Yuri Manin on the occasion of his 70th birthday \\ O simili o dissimili che sieno questi mondi \\ non con minor raggione sarebe bene a l'uno \\ l'essere che a l'altro \\ Giordano Bruno - De l'infinito, universo e mondi
}

Contents

1. Introduction

2. A look at the Weil proof

2.1. Correspondences and divisors 7

2.2. The explicit formula 8

2.3. Riemann-Roch and positivity

2.4. A tentative dictionary 12

3. Quantum statistical mechanics and arithmetic 12

3.1. The Bost-Connes endomotive 14

3.2. Scaling as Frobenius in characteristic zero 15

4. The adeles class space 16

4.1. Cyclic module 17

4.2. The restriction map 18

4.3. The Morita equivalence and cokernel for $\mathbb{K}=\mathbb{Q} \quad 19$

4.4. The cokernel of $\rho$ for general global fields 21

4.5. Trace pairing and vanishing 25

5. Primitive cohomology 25

6. A cohomological Lefschetz trace formula 27

6.1. Weil's explicit formula as a trace formula 27

6.2. Weil Positivity and the Riemann Hypothesis 28

7. Correspondences 30

7.1. The scaling correspondence as Frobenius 30

7.2. Fubini's theorem and the trivial correspondences 32

8. Thermodynamics and geometry of the primes 33

8.1. The global Morita equivalence 34

8.2. The valuation systems 36

\begin{tabular}{|l|l|}
\hline 8.3. The curve inside the adeles class space & 39 \\
\hline
\end{tabular}

8.4. The valuation systems for $\mathbb{K}=\mathbb{Q}$ 
8.5. The cyclic covering $\tilde{\Xi}_{\mathbb{Q}}$ of $\Xi_{\mathbb{Q}} \quad 47$

8.6. Arithmetic subalgebra, Frobenius and monodromy 48

9. Functoriality of the adeles class space 50

9.1. The norm map 51

9.2. The Weil group and the transfer map

9.3. The covering 52

9.4. The function field case 54

10. Vanishing cycles: an analogy 55

10.1. Two real places 5

10.2. A real and a non-archimedean place 5

10.3. Singularities of maps $\quad 59$

References

\section{INTRODUCTION}

This paper explores analogies between the Weil proof of the Riemann Hypothesis for function fields and the geometry of the adeles class space, which is the noncommutative space underlying the spectral realization of the zeros of the Riemann zeta function constructed in [10. Our purpose is to build a dictionary between the algebro-geometric setting of algebraic curves, divisors, the Riemann-Roch formula, and the Frobenius map, around which the Weil proof is built, and the world of noncommutative spaces, cyclic cohomology and KK-theory, index formulae, and the thermodynamical notions of quantum statistical mechanics, which, as we already argued in [11], provide an analog of the Frobenius in characteristic zero via the scaling action on the dual system.

The present work builds upon several previous results. The first input is the spectral realization of [10], where the adeles class space was first identified as the natural geometric space underlying the Riemann zeta function, where the Weil explicit formula acquires an interpretation as a trace formula. In [10] the analytic setting is that of Hilbert spaces, which provide the required positivity, but the spectral realization only involves the critical zeros. In [11], we provided a cohomological interpretation of the trace formula, using cyclic homology. In the setting of [11, the analysis is as developed by Ralph Meyer in 32 and uses spaces of rapidly decaying functions instead of Hilbert spaces. In this case, all zeros contribute to the trace formula, and the Riemann Hypothesis becomes equivalent to a positivity question. This mirrors more closely the structure of the two main steps in the Weil proof, namely the explicit formula and the positivity $\operatorname{Tr}\left(Z * Z^{\prime}\right)>$ 0 for correspondences (see below). The second main building block we need to use is the theory of endomotives and their quantum statistical mechanical properties we studied in [11]. Endomotives are a pseudo-abelian category of noncommutative spaces that naturally generalize the category of Artin motives. They are built from semigroup actions on projective limits of Artin motives. The morphisms in the category of endomotives generalize the notion of correspondence given by algebraic cycles in the product used in the theory of motives to the 
setting of étale groupoids, to account naturally for the presence of the semigroup actions. Endomotives carry a Galois action inherited from Artin motives and they have both an algebraic and an analytic manifestation. The latter provides the data for a quantum statistical mechanical system, via the natural time evolution associated by Tomita's theory to a probability measure carried by the analytic endomotive. The main example that is of relevance to the Riemann zeta function is the endomotive underlying the Bost-Connes quantum statistical mechanical system of [5]. One can pass from a quantum statistical mechanical system to the "dual system" (in the sense of the duality of type III and type II factors in [6], [36]), which comes endowed with a scaling action induced by the time evolution. A general procedure described in [11] shows that there is a "restriction map" (defined as a morphism in the abelian category of modules over the cyclic category) from the dual system to a line bundle over the space of low temperature KMS states of the quantum statistical mechanical system. The cokernel of this map is not defined at the level of algebras, but it makes sense in the abelian category and carries a corresponding scaling action. We argued in [1] that the induced scaling action on the cyclic homology of this cokernel may be thought of as an analog of the action of Frobenius on étale cohomology. This claim is justified by the role that this scaling action of $\mathbb{R}_{+}^{*}$, combined with the action of $\hat{\mathbb{Z}}^{*}$ carried by the Bost-Connes endomotive, has in the trace formula, see [10, [11] and $\S 4$ of 13. Further evidence for the role of the scaling action as Frobenius is given in [20], where it is shown that, in the case of function fields, for a natural quantum statistical mechanical system that generalizes the Bost-Connes system to rank one Drinfeld modules, the scaling action on the dual system can be described in terms of the Frobenius and inertia groups.

In the present paper we continue along this line of thought. We begin by reviewing the main steps in the Weil proof for function fields, where we highlight the main conceptual steps and the main notions that will need an analog in the noncommutative geometry setting. We conclude this part by introducing the main entries in our still tentative dictionary. The rest of the paper discusses in detail some parts of the dictionary and provides evidence in support of the proposed comparison. We begin this part by recalling briefly the properties of the Bost-Connes endomotive from [11] followed by the description of the "restriction map" corresponding to the inclusion of the ideles class group $C_{\mathbb{K}}=\mathbb{A}_{\mathbb{K}}^{*} / \mathbb{K}^{*}$ in the noncommutative adeles class space $X_{\mathbb{K}}=\mathbb{A}_{\mathbb{K}} / \mathbb{K}^{*}$. We discuss its relation to the exact sequence of Hilbert spaces of [10] that plays a crucial role in obtaining the spectral realization as an "absorption spectrum".

We then concentrate on the geometry of the adeles class space over an arbitrary global field and the restriction map in this general setting, viewed as a map of cyclic modules. We introduce the actions $\vartheta_{a}$ and $\vartheta_{m}$ (with $a$ and $m$ respectively for additive and multiplicative) of $\mathbb{A}_{\mathbb{K}}^{*}$ on suitable function spaces on $\mathbb{A}_{\mathbb{K}}$ and on $C_{\mathbb{K}}$ and the induced action on the cokernel of the restriction map in the category of cyclic modules. We prove the corresponding general form of the associated Lefschetz trace formula, as a cohomological reformulation of the trace formula of [10] using the analytical setting of 32]. 
The form of the trace formula and the positivity property that is equivalent, in this setting, to the Riemann Hypothesis for the corresponding $L$-functions with Grössencharakter, suggest by comparison with the analogous notions in the Weil proof a natural candidate for the analog of the Frobenius correspondence on the curve. This is given by the graph of the scaling action. We can also identify the analog of the degree and co-degree of a correspondence, and the analog of the self intersection of the diagonal on the curve, by looking at the explicit form of our Lefschetz trace formula. We also have a clear analog of the first step in the Weil proof of positivity, which consists of adjusting the degree by multiples of the trivial correspondences. This step is possible, with our notion of correspondences, due to a subtle failure of Fubini's theorem that allows us to modify the degree by adding elements in the range of the "restriction map", which play in this way the role of the trivial correspondences. This leaves open the more difficult question of identifying the correct analog of the principal divisors, which is needed in order to continue the dictionary.

We then describe how to obtain a good analog of the algebraic points of the curve in the number field case (in particular in the case of $\mathbb{K}=\mathbb{Q}$ ), in terms of the thermodynamical properties of the system. This refines the general procedure described in [11. In fact, after passing to the dual system, one can consider the periodic orbits. We explain how, by the result of [10, these are the noncommutative spaces where the geometric side of the Lefschetz trace formula concentrates. We show that, in turn, these periodic orbits carry a time evolution and give rise to quantum statistical mechanical systems, of which one can consider the low temperature KMS states. To each periodic orbit one can associate a set of "classical points" and we show that these arise as extremal low temperature KMS states of the corresponding system. We show that, in the function field case, the space obtained in this way indeed can be identified, compatibly with the Frobenius action, with the algebraic points of the curve, albeit by a non-canonical identification. Passing to the dual system is the analog in characteristic zero of the transition from $\mathbb{F}_{q}$ to its algebraic closure $\overline{\mathbb{F}}_{q}$. Thus, the procedure of considering periodic orbits in the dual system and classical points of these periodic orbits can be seen as an analog, for our noncommutative space, of considering points defined over the extensions $\mathbb{F}_{q^{n}}$ of $\mathbb{F}_{q}$ in the case of varieties defined over finite fields ( $c f$. [11] and $\S 4$ of [13]).

We analyze the behavior of the adeles class space under field extensions and the functoriality question. We then finish the paper by sketching an analogy between some aspects of the geometry of the adeles class spaces and the theory of singularities, which may be useful in adapting to this context some of the techniques of vanishing and nearby cycles.

\section{A LOOK AT ThE WeIL PROOF}

In this preliminary section, we briefly review some aspects of the Weil proof of the Riemann Hypothesis for function fields, with an eye on extending some of the 
basic steps and concept to a noncommutative framework, which is what we will be doing in the rest of the paper.

In this section we let $\mathbb{K}$ be a global field of positive characteristic $p>0$. One knows that, in this case, there exists a smooth projective curve over a finite field $\mathbb{F}_{q}$, with $q=p^{r}$ for some $r \in \mathbb{N}$, such that

$$
\mathbb{K}=\mathbb{F}_{q}(C)
$$

is the field of functions of $C$. For this reason, a global field of positive characteristic is called a function field.

We denote by $\Sigma_{\mathbb{K}}$ the set of places of $\mathbb{K}$. A place $v \in \Sigma_{\mathbb{K}}$ is a Galois orbit of points of $C\left(\overline{\mathbb{F}}_{q}\right)$. The degree $n_{v}=\operatorname{deg}(v)$ is its cardinality, namely the number of points in the orbit of the Frobenius acting on the fiber of the natural map from points to places

$$
C\left(\overline{\mathbb{F}}_{q}\right) \rightarrow \Sigma_{\mathbb{K}}
$$

This means that the fiber over $v$ consists of $n_{v}$ conjugate points defined over $\mathbb{F}_{q^{n_{v}}}$, the residue field of the local field $\mathbb{K}_{v}$.

The curve $C$ over $\mathbb{F}_{q}$ has a zeta function of the form

$$
Z_{C}(T)=\exp \left(\sum_{n=1}^{\infty} \frac{\# C\left(\mathbb{F}_{q^{n}}\right)}{n} T^{n}\right),
$$

with $\log Z_{C}(T)$ the generating function for the number of points of $C$ over the fields $\mathbb{F}_{q^{n}}$. It is customary to use the notation

$$
\zeta_{\mathbb{K}}(s)=\zeta_{C}(s)=Z_{C}\left(q^{-s}\right) .
$$

It converges for $\Re(s)>1$. In terms of Euler product expansions one writes

$$
\zeta_{\mathbb{K}}(s)=\prod_{v \in \Sigma_{\mathbb{K}}}\left(1-q^{-n_{v} s}\right)^{-1} .
$$

In terms of divisors of $C$, one has equivalently

$$
\zeta_{\mathbb{K}}(s)=\zeta_{C}(s)=\sum_{D \geq 0} N(D)^{-s}
$$

where the norm of the divisor $D$ is $N(D)=q^{\operatorname{deg}(D)}$.

The Riemann-Roch formula for the curve $C$ states that

$$
\ell(D)-\ell\left(\kappa_{C}-D\right)=\operatorname{deg}(D)-g+1,
$$

where $\kappa_{C}$ is the canonical divisor on $C$, with degree $\operatorname{deg}\left(\kappa_{C}\right)=2 g-2$ and $h^{0}\left(\kappa_{C}\right)=g$, and $\ell(D)$ the dimension of $H^{0}(D)$. Both $\operatorname{deg}(D)$ and $N(D)$ are well defined on the equivalence classes obtained by adding principal divisors, that is,

$$
D \sim D^{\prime} \Longleftrightarrow D-D^{\prime}=(f),
$$

for some $f \in \mathbb{K}^{*}$. The Riemann-Roch formula (2.7) also implies that the zeta function $\zeta_{\mathbb{K}}(s)$ satisfies the functional equation

$$
\zeta_{\mathbb{K}}(1-s)=q^{(1-g)(1-2 s)} \zeta_{\mathbb{K}}(s) .
$$


The zeta function $\zeta_{\mathbb{K}}(s)$ can also be written as a rational function

$$
Z_{C}(T)=\frac{P(T)}{(1-T)(1-q T)}, \quad T=q^{-s},
$$

where $P(T)$ is a polynomial of degree $2 g$ and integer coefficients

$$
P(T)=\prod_{j=1}^{2 g}\left(1-\lambda_{j} T\right)
$$

In particular, one has

$$
\# C\left(\mathbb{F}_{q^{n}}\right)=q^{n}+1-\sum_{j=1}^{2 g} \lambda_{j} .
$$

Another important reformulation of the zeta function can be given in terms of étale cohomology. Namely, the coefficients $\# C\left(\mathbb{F}_{q^{n}}\right)$ that appear in the zeta function can be rewritten as

$$
\# C\left(\mathbb{F}_{q^{n}}\right)=\# \operatorname{Fix}\left(\operatorname{Fr}^{n}: \bar{C} \rightarrow \bar{C}\right),
$$

with $\bar{C}=C \otimes_{\mathbb{F}_{q}} \overline{\mathbb{F}}_{q}$. The Lefschetz fixed point formula for étale cohomology then shows that

$$
\# C\left(\mathbb{F}_{q^{n}}\right)=\sum_{i=0}^{2}(-1)^{i} \operatorname{Tr}\left(\operatorname{Fr}^{n} \mid H_{e t}^{i}\left(\bar{C}, \mathbb{Q}_{\ell}\right)\right) .
$$

Thus, the zeta function can be written in the form

$$
\zeta_{\mathbb{K}}(s)=\prod_{i=0}^{2}\left(\exp \left(\sum_{n=1}^{\infty} \operatorname{Tr}\left(\operatorname{Fr}^{n} \mid H_{e t}^{i}\left(\bar{C}, \mathbb{Q}_{\ell}\right)\right) \frac{q^{-s n}}{n}\right)\right)^{(-1)^{i}} .
$$

The analog of the Riemann hypothesis for the zeta functions $\zeta_{\mathbb{K}}(s)$ of function fields was stated in 1924 by E. Artin as the property that the zeros lie on the line $\Re(s)=1 / 2$. Equivalently, it states that the complex numbers $\lambda_{j}$ of (2.11), which are are the eigenvalues of the Frobenius acting on $H_{e t}^{1}\left(\bar{C}, \mathbb{Q}_{\ell}\right)$, are algebraic numbers satisfying

$$
\left|\lambda_{j}\right|=\sqrt{q}
$$

The Weil proof can be formulated either using étale cohomology, or purely in terms of the Jacobian of the curve, or again (equivalently) in terms of divisors on $C \times C$. We follow this last viewpoint and we recall in detail some of the main steps in the proof. 
2.1. Correspondences and divisors. Correspondences $Z$, given by (non-vertical) divisors on $C \times C$, form a ring under composition

$$
Z_{1} \star Z_{2}=\left(p_{13}\right)_{*}\left(p_{12}^{*} Z_{1} \bullet p_{23}^{*} Z_{2}\right),
$$

with $p_{i j}: C \times C \times C \rightarrow C \times C$ the projections, and $\bullet$ the intersection product. The ring has an involution

$$
Z^{\prime}=\sigma(Z)
$$

where $\sigma$ is the involution that exchanges the two copies of $C$ in the product $C \times C$. The degree $d(Z)$ and the codegree $d^{\prime}(Z)$ are defined as the intersection numbers

$$
d(Z)=Z \bullet(P \times C) \quad \text { and } \quad d^{\prime}(Z)=Z \bullet(C \times P), \quad \forall P \in C .
$$

They satisfy the relations

$$
d\left(Z^{\prime}\right)=d^{\prime}(Z), \quad \text { and } \quad d\left(Z_{1} \star Z_{2}\right)=d\left(Z_{1}\right) d\left(Z_{2}\right) .
$$

The correspondences $P \times C$ and $C \times P$ are called trivial correspondences. One can consider the abelian group $\operatorname{Div}_{t r}(C \times C)$ generated by these trivial correspondence and take the quotient

$$
\mathcal{C}(C):=\operatorname{Div}(C \times C) / \operatorname{Div}_{t r}(C \times C) .
$$

It is always possible to change the degree and codegree of a correspondence $Z$ by adding a multiple of the trivial correspondences $P \times C$ and $C \times P$, so that, for any element in $\mathcal{C}$ we find a representative $Z \in$ Corr with

$$
d(Z)=d^{\prime}(Z)=0 .
$$

One also wants to consider correspondences up to linear equivalence,

$$
Z_{1} \sim Z_{2} \Longleftrightarrow Z_{1}-Z_{2}=(f),
$$

where $(f)$ is a principal divisor on $C \times C$. Thus, one can consider

$$
\operatorname{Pic}(C \times C)=\operatorname{Div}(C \times C) / \sim
$$

and its quotient $\mathcal{P}(C)$ modulo the classes of the trivial correspondences.

A correspondence $Z$ is effective if it is given by an effective divisor on $C \times C$, namely if it is a combination $Z=\sum_{i} n_{i} Z_{i}$ of curves $Z_{i} \subset C \times C$ with coefficients $n_{i} \geq 0$. We write $Z \geq 0$ to mean its effectiveness. An effective correspondence $Z \geq 0$ that is nonempty can be viewed as a multivalued map

$$
Z: C \rightarrow C, \quad P \mapsto Z(P),
$$

with $Z(P)=\operatorname{proj}_{C}(Z \bullet(P \times C))$, of which the divisor $Z \subset C \times C$ is the graph, and with the product (2.17) given by the composition.

The trace of a correspondence $Z$ on $C \times C$ is the expression

$$
\operatorname{Tr}(Z)=d(Z)+d^{\prime}(Z)-Z \bullet \Delta,
$$

with $\Delta$ the diagonal (identity correspondence) and $\bullet$ the intersection product. This is well defined on $\mathcal{P}(C)$ since $\operatorname{Tr}(Z)=0$ for principal divisors and trivial correspondences. 
Consider a correspondence of degree $g$ of the form $Z=\sum a_{n} \mathrm{Fr}^{n}$, given by a combination of powers of the Frobenius. Then $Z$ can be made effective by adding a principal correspondence which is defined over $\mathbb{F}_{q}$ and which commutes with Fr.

This can be seen as follows. The Riemann-Roch theorem ensures that one can make $Z$ effective by adding a principal correspondence, over the field $k(P)$, where $k$ is the common field of definition of the correspondence $Z$ and of the curve $(c f$. [34]) and $P$ is a generic point. A correspondence of the form $Z=\sum a_{n} \mathrm{Fr}^{n}$ is in fact defined over $\mathbb{F}_{q}$ hence the principal correspondence is also defined over $\mathbb{F}_{q}$. As such it automatically commutes with Fr (cf. [37], p. 287).

Notice however that, in general, it is not possible to modify a divisor $D$ of degree one on $C$ to an effective divisor in such a way that the added pricipal divisor has support on the same Frobenius orbit. An illustrative example is given in Chapter 4 of [13].

2.2. The explicit formula. The main steps in the Weil proof of RH for function fields are

(1) The explicit formula

(2) Positivity

Let $\mathbb{K}$ be a global field and let $\mathbb{A}_{\mathbb{K}}$ denote its ring of adeles. Let $\Sigma_{\mathbb{K}}$ denote the set of places of $\mathbb{K}$. Let $\alpha$ be a non-trivial character of $\mathbb{A}_{\mathbb{K}}$ which is trivial on $\mathbb{K} \subset \mathbb{A}_{\mathbb{K}}$. We write

$$
\alpha=\prod_{v \in \Sigma_{\mathbb{K}}} \alpha_{v}
$$

for the decomposition of $\alpha$ as a product of its restrictions to the local fields $\alpha_{v}=\left.\alpha\right|_{\mathbb{K}_{v}}$.

Consider the bicharacter

$$
\langle z, \lambda\rangle:=\lambda^{z}, \quad \text { for }(z, \lambda) \in \mathbb{C} \times \mathbb{R}_{+}^{*} .
$$

Let $N$ denote the range of the norm $|\cdot|: C_{\mathbb{K}} \rightarrow \mathbb{R}_{+}^{*}$. Then $N^{\perp} \subset \mathbb{C}$ denotes the subgroup

$$
N^{\perp}:=\left\{z \in \mathbb{C} \mid \lambda^{z}=1, \forall \lambda \in N\right\} .
$$

Consider then the expression

$$
\sum_{\rho \in \mathbb{C} / N^{\perp} \mid L(\tilde{\chi}, \rho)=0} \hat{f}(\tilde{\chi}, \rho),
$$

with $L(\tilde{\chi}, \rho)$ the $L$-function with Grössencharakter $\chi$, where $\tilde{\chi}$ denotes the extension to $C_{\mathbb{K}}$ of the character $\chi \in \widehat{C_{\mathbb{K}, 1}}$, the Pontrjagin dual of $C_{\mathbb{K}, 1}$. Here $\hat{f}(\tilde{\chi}, \rho)$ denotes the Fourier transform

$$
\hat{f}(\tilde{\chi}, \rho)=\int_{C_{\mathbb{K}}} f(u) \tilde{\chi}(u)|u|^{\rho} d^{*} u
$$

of a test function $f$ in the Schwartz space $\mathcal{S}\left(C_{\mathbb{K}}\right)$. 
In the case where $N=q^{\mathbb{Z}}$ (function fields), the subgroup $N^{\perp}$ is nontrivial and given by

$$
N^{\perp}=\frac{2 \pi i}{\log q} \mathbb{Z}
$$

Since in the function field case the $L$-fuctions are functions of $q^{-s}$, there is a periodicity by $N^{\perp}$, hence we need to consider only $\rho \in \mathbb{C} / N^{\perp}$. In the number field case this does not matter, since $N=\mathbb{R}_{+}^{*}$ and $N^{\perp}$ is trivial.

The Weil explicit formula is the remarkable identity 42

$$
\hat{h}(0)+\hat{h}(1)-\sum_{\rho \in \mathbb{C} / N^{\perp} \mid L(\tilde{\chi}, \rho)=0} \hat{h}(\tilde{\chi}, \rho)=\sum_{v \in \Sigma_{\mathbb{K}}} \int_{\left(\mathbb{K}_{v}^{*}, \alpha_{v}\right)}^{\prime} \frac{h\left(u^{-1}\right)}{|1-u|} d^{*} u .
$$

Here the Fourier transform $\hat{h}$ is as in (2.30). The test function $h$ has compact support and belongs to the Schwartz space $\mathcal{S}\left(C_{\mathbb{K}}\right)$. As soon as $h(1) \neq 0$ the integrals in the right hand side are singular so that one needs to specify how to take their principal value. This was done in [42] and it was shown in [10] that the same principal value can in fact be defined in the following unified way.

Definition 2.1. For a local field $K$ and a given (non-trivial) additive character $\beta$ of $K$, one lets $\varrho_{\beta}$ denote the unique distribution extending $d^{*} u$ at $u=0$, whose Fourier transform

$$
\hat{\varrho}(y)=\int_{K} \varrho(x) \beta(x y) d x
$$

satisfies the vanishing condition $\hat{\varrho}(1)=0$.

Then by definition the principal value $\int^{\prime}$ is given by

$$
\int_{(K, \beta)}^{\prime} \frac{f\left(u^{-1}\right)}{|1-u|} d^{*} u=\left\langle\varrho_{\beta}, g\right\rangle, \quad \text { with } \quad g(\lambda)=\frac{f\left((\lambda+1)^{-1}\right)}{|\lambda+1|},
$$

where $\left\langle\varrho_{\beta}, g\right\rangle$ denotes the pairing of the distribution $\varrho_{\beta}$ and the function $g(\lambda)$. This makes sense provided the support of $f$ is compact which implies that $g(\lambda)$ vanishes identically in a neighborhood of $\lambda=-1$.

The Weil explicit formula is a far reaching generalization of the relation between primes and zeros of the Riemann zeta function, originally due to Riemann 33 .

2.3. Riemann-Roch and positivity. Weil positivity is the statement that, if $Z$ is a nontrivial correspondence in $\mathcal{P}(C)$ (i.e. as above a correspondence on $C \times C$ modulo trivial ones and up to linear equivalence), then

$$
\operatorname{Tr}\left(Z \star Z^{\prime}\right)>0 .
$$

This is proved using the Riemann-Roch formula on $C$ to show that one can achieve effectivity. In fact, using trivial correspondences to adjust the degree one can assume that $d(Z)=g$. Then the Riemann-Roch formula (2.7) shows that if $D$ is a divisor on $C$ of degree $\operatorname{deg}(D)=g$ then there are effective representatives 
in the linear equivalence class of $D$. The intersection of $Z \subset C \times C$ with $P \times C$ defines a divisor $Z(P)$ on $C$ with

$$
\operatorname{deg}(Z(P))=d(Z)=g .
$$

Thus, the argument above shows that there exists $f_{P} \in \mathbb{K}^{*}$ such that $Z(P)+\left(f_{P}\right)$ is effective. This determines an effective divisor $Z+(f)$ on $C \times C$. Thus, we can assume that $Z$ is effective, hence we can write it as a multivalued function

$$
P \mapsto Z(P)=Q_{1}+\cdots+Q_{g} .
$$

The product $Z \star Z^{\prime}$ is of the form

$$
Z \star Z^{\prime}=d^{\prime}(Z) \Delta+Y,
$$

where $\Delta$ is the diagonal in $C \times C$ and $Y$ is the effective correspondence such that $Y(P)$ is the divisor on $C$ given by the sum of points in

$$
\left\{Q \in C \mid Q=Q_{i}(P)=Q_{j}(P), i \neq j\right\} .
$$

One sees this from the description in terms of intersection product that it is given by the multivalued function

$$
\left(Z \star Z^{\prime}\right)(Q)=\sum_{i, j} \sum_{P \in \mathcal{U}_{i j}(Q)} P,
$$

where

$$
\mathcal{U}_{i j}(Q)=\left\{P \in C \mid Q_{i}(P)=Q_{j}(P)=Q\right\} .
$$

One can separate this out in the contribution of the locus where $Q_{i}=Q_{j}$ for $i \neq j$ and the part where $i=j$,

$$
\left(Z \star Z^{\prime}\right)(Q)=U(Q)+Y(Q) .
$$

Notice that

$$
\#\left\{P \in C \mid Q=Q_{i}(P), \text { for some } i=1, \ldots, g\right\}=d^{\prime}(Z) .
$$

Thus, for $i=j$ we obtain that the divisor $U(Q)=\sum_{i} \sum_{P \in \mathcal{U}_{i i}(Q)} P$ is just $d^{\prime}(Z) \Delta(Q)$, while for $i \neq j$ one obtains the remaining term $Y$ of (2.37).

In the case $g=1$, the effective correspondence $Z(P)=Q(P)$ is single valued and the divisor $\left(Z \star Z^{\prime}\right)(P)$ of (2.38) reduces to the sum of points in

$$
\mathcal{U}(Q)=\{P \in C \mid Q(P)=Q\} .
$$

There are $d^{\prime}(Z)$ such points so one obtains

$$
Z \star Z^{\prime}=d^{\prime}(Z) \Delta, \quad \text { with } \quad \operatorname{Tr}\left(Z \star Z^{\prime}\right)=2 d^{\prime}(Z) \geq 0,
$$

since for $g=1$ one has $\Delta \bullet \Delta=0$ and $d^{\prime}(Z) \geq 0$ since $Z$ is effective.

In the case of genus $g>1$, the Weil proof proceeds as follows. Let $\kappa_{C}$ be a choice of an effective canonical divisor for $C$ without multiple points, and let $\left\{f_{1}, \ldots, f_{g}\right\}$ be a basis of the space $H^{0}\left(\kappa_{C}\right)$. One then considers the function $C \rightarrow M_{g \times g}\left(\mathbb{F}_{q}\right)$ to $g \times g$ matrices

$$
P \mapsto M(P), \quad \text { with } \quad M_{i j}(P)=f_{i}\left(Q_{j}(P)\right) .
$$


and the function $K: C \rightarrow \mathbb{F}_{q}$ given by

$$
K(P)=\operatorname{det}(M(P))^{2} .
$$

The function $P \mapsto K(P)$ of (2.42) is a rational function with $(2 g-2) d^{\prime}(Z)$ double poles. In fact, $K(P)$ is a symmetric function of the $Q_{j}(P)$, because of the squaring of the determinant. The composition $P \mapsto\left(Q_{j}(P)\right) \mapsto K(P)$ is then a rational function of $P \in C$. The poles occur (as double poles) at those points $P \in C$ for which some $Q_{i}(P)$ is a component of $\kappa_{C}$. The canonical divisor $\kappa_{C}$ has degree $2 g-2$. This means that there are $(2 g-2) d^{\prime}(Z)$ such double poles.

For $Z \star Z^{\prime}=d^{\prime}(Z) \Delta+Y$ as above, the intersection number $Y \bullet \Delta$ satisfies the estimate

$$
Y \bullet \Delta \leq(4 g-4) d^{\prime}(Z) .
$$

In fact, the rational function $K(P)$ of (2.42) has a number of zeros equal to $(4 g-4) d^{\prime}(Z)$. On the other hand, $Y \bullet \Delta$ counts the number of times that $Q_{i}=Q_{j}$ for $i \neq j$. Since each point $P$ with $Q_{i}(P)=Q_{j}(P)$ for $i \neq j$ produces a zero of $K(P)$, one sees that $Y \bullet \Delta$ satisfies the estimate (2.43). Notice that, for genus $g>1$ the self intersection of the diagonal is the Euler characteristic

$$
\Delta \bullet \Delta=2-2 g=\chi(C) .
$$

Moreover, we have

$$
d\left(Z \star Z^{\prime}\right)=d(Z) d^{\prime}(Z)=g d^{\prime}(Z)=d^{\prime}\left(Z \star Z^{\prime}\right) .
$$

Thus, using again the decomposition (2.37) and the definition of the trace of a correspondence (2.25), together with (2.44) and (2.45) one obtains

$$
\begin{aligned}
& \operatorname{Tr}\left(Z \star Z^{\prime}\right)=2 g d^{\prime}(Z)+(2 g-2) d^{\prime}(Z)-Y \bullet \Delta \\
& \geq(4 g-2) d^{\prime}(Z)-(4 g-4) d^{\prime}(Z)=2 d^{\prime}(Z) \geq 0 .
\end{aligned}
$$

This gives the positivity (2.35).

In the Weil proof of $\mathrm{RH}$ for function fields, one concentrates on a particular type of correspondences, namely those that are of the form

$$
Z_{n, m}=m \Delta+n \mathrm{Fr},
$$

for $n, m \in \mathbb{Z}$, with Fr the Frobenius correspondence.

Notice that, while the correspondence depends linearly on $n, m \in \mathbb{Z}$, the expression for the trace gives

$$
\operatorname{Tr}\left(Z_{n, m} \star Z_{n, m}^{\prime}\right)=2 g m^{2}+2(1+q-N) m n+2 g q n^{2},
$$

where $N=\# C\left(\mathbb{F}_{q}\right)$. In particular, (2.48) depends quadratically on $(n, m)$. In the process of passing from a correspondence of degree $g$ to an effective correspondence, this quadratic dependence on $(n, m)$ is contained in the multiplicity $d^{\prime}(Z)$. Notice, moreover, that the argument does not depend on the torsion part of the ring of correspondences. 
2.4. A tentative dictionary. In the rest of the paper we illustrate some steps towards the creation of a dictionary relating the main steps in the Weil proof described above to the noncommutative geometry of the adeles class space of a global field. The noncommutative geometry approach has the advantage that it provides (see [10, [32], 11]) a Lefschetz trace formula interpretation for the Weil explicit formula and that it gives a parallel formulation for both function fields and number fields. Parts of the dictionary sketched below are very tentative at this stage, so we mostly concentrate, in the rest of the paper, on illustrating what we put in the first few lines of the dictionary, on the role of the scaling correspondence as Frobenius and its relation to the explicit formula.

\begin{tabular}{|c|c|}
\hline Frobenius correspondence & $Z(f)=\int_{C_{\mathbb{K}}} f(g) Z_{g} d^{*} g$ \\
\hline Trivial correspondences & Elements of the range $\mathcal{V}$ \\
\hline $\begin{array}{l}\text { Adjusting the degree } \\
\text { by trivial correspondences }\end{array}$ & $\begin{array}{l}\text { Fubini step } \\
\text { on the test functions }\end{array}$ \\
\hline Correspondences & Bivariant elements $Z(f) \Rightarrow \Gamma(f)$ \\
\hline Degree of a correspondence & Pointwise index \\
\hline Riemann-Roch & Index theorem \\
\hline Effective correspondences & Epimorphism of $C^{*}$-modules \\
\hline $\operatorname{deg} Z(P) \geq g \Rightarrow Z+(f)$ effective & $d(\Gamma)>0 \Rightarrow \Gamma+K$ onto \\
\hline Lefschetz formula & $\begin{array}{l}\text { Bivariant Chern of } \Gamma(f) \\
\text { (by localization on the graph } Z(f) \text { ) }\end{array}$ \\
\hline
\end{tabular}

\section{Quantum Statistical Mechanics And ARITHmetic}

The work of Bost-Connes 5 first revealed the presence of an interesting interplay between quantum statistical mechanics and Galois theory. More recently, several 
generalizations [12, [14, [15, [11], 20], 25], 26] have confirmed and expanded this viewpoint. The general framework of interactions between noncommutative geometry and number theory described in [29, 30, [13, 31] recast these phenomena into a broader picture, of which we explore in this paper but one of many facets.

The basic framework that combines quantum statistical mechanics and Galois theory can be seen as an extension, involving noncommutative spaces, of the category of Artin motives. In the setting of pure motives (see [27]), Artin motives correspond to the subcategory generated by zero dimensional objects, with morphisms given by algebraic cycles in the product (in this case without the need to specify with respect to which equivalence relation). Endomotives were introduced in [11] as noncommutative spaces of the form

$$
\mathcal{A}_{\mathbb{K}}=A \rtimes S,
$$

where $A$ is an inductive limit of reduced finite dimensional commutative algebras over the field $\mathbb{K}$, i.e. a projective limit of Artin motives, and $S$ is a unital abelian semigroup of algebra endomorphisms $\rho: A \rightarrow A$. These have the following properties: the algebra $A$ is unital; the image $e=\rho(1) \in A$ is an idempotent, for all $\rho \in S$; each $\rho \in S$ is an isomorphism of $A$ with the compressed algebra $e A e$. A general construction given in [1] based on self maps of algebraic varieties provides a large class of examples over different fields $\mathbb{K}$. We are mostly interested here in the case where $\mathbb{K}$ is a number field and for part of our discussion below we will concentrate on a special case (the Bost-Connes endomotive) over the field $\mathbb{K}=\mathbb{Q}$.

Endomotives form a pseudo-abelian category where morphisms are correspondences given by $\mathcal{A}_{\mathbb{K}}-\mathcal{B}_{\mathbb{K}}$-bimodules that are finite and projective as right modules. These define morphisms in the additive KK-category and in the abelian category of cyclic modules. In fact, in addition to the algebraic form described above, endomotives also have an analytic structure given by considering, instead of the $\mathbb{K}$-algebra (3.1) the $C^{*}$-algebra

$$
C(X) \rtimes S,
$$

where $X$ denotes the totally disconnected Hausdorff space $X=X(\overline{\mathbb{K}})$ of algebraic points of the projective limit of Artin motives. There is a canonical action of the Galois group $G=\operatorname{Gal}(\overline{\mathbb{K}} / \mathbb{K})$ by automorphisms of the $C^{*}$-algebra (3.2) globally preserving $C(X)$. We refer the reader to [11 for a more detailed discussion of algebraic and analytic endomotives and the properties of morphisms in the corresponding categories.

If the endomotive is "uniform" in the sense specified in [11, the space $X$ comes endowed with a probability measure $\mu$ that induces a state $\varphi$ on the $C^{*}$-algebra (3.2). The general Tomita theory of modular automorphism groups in the context of von Neumann algebras [35] shows that there is a natural time evolution for which the state $\varphi$ is $\mathrm{KMS}_{1}$. One can then consider the set $\Omega_{\beta}$ of low temperature KMS states for the resulting quantum statistical mechanical system. One also associates to the system $(\mathcal{A}, \sigma)$ of the $C^{*}$-algebra with the time evolution its dual 
system $(\hat{\mathcal{A}}, \theta)$, where the algebra $\hat{\mathcal{A}}=\mathcal{A} \rtimes_{\sigma} \mathbb{R}$ is obtained by taking the crossed product with the time evolution and $\theta$ is the scaling action of $\mathbb{R}_{+}^{*}$

$$
\theta_{\lambda}\left(\int x(t) U_{t} d t\right)=\int \lambda^{i t} x(t) U_{t} d t
$$

One then constructs an $\mathbb{R}_{+}^{*}$ equivariant map

$$
\pi: \hat{\mathcal{A}}_{\beta} \rightarrow C\left(\tilde{\Omega}_{\beta}, \mathcal{L}^{1}\right),
$$

from a suitable subalgebra $\hat{\mathcal{A}}_{\beta} \subset \hat{\mathcal{A}}$ of the dual system to functions on a principal $\mathbb{R}_{+}^{*}$-bundle $\tilde{\Omega}_{\beta}$ over the low temperature KMS states of the system, with values in trace class operators. Since traces define morphisms in the cyclic category, the map (3.4) can be used to construct a morphism $\delta=(\operatorname{Tr} \circ \pi)^{\natural}$ at the level of cyclic modules

$$
\hat{\mathcal{A}}_{\beta}^{\natural} \stackrel{(\operatorname{Tr} \circ \pi)^{\natural}}{\longrightarrow} C\left(\tilde{\Omega}_{\beta}\right)^{\natural} .
$$

This map can be loosely thought of as a "restriction map" corresponding to the inclusion of the "classical points" in the noncommutative space. One can then consider the cokernel of this map in the abelian category of cyclic modules. In [11] we denoted the procedure described above "cooling and distillation" of endomotives. We refer the reader to [11] for the precise technical hypotheses under which this procedure can be performed. Here we only gave an impressionistic sketch aimed at recalling briefly the main steps involved.

3.1. The Bost-Connes endomotive. The main example of endomotive we will consider here in relation to the geometry of the adeles class space is the Bost-Connes system. This can be constructed as an endomotive over $\mathbb{K}=\mathbb{Q}$, starting from the projective system $X_{n}=\operatorname{Spec}\left(A_{n}\right)$, with $A_{n}=\mathbb{Q}[\mathbb{Z} / n \mathbb{Z}]$ the group ring of $\mathbb{Z} / n \mathbb{Z}$. The inductive limit is the group $\operatorname{ring} A=\mathbb{Q}[\mathbb{Q} / \mathbb{Z}]$ of $\mathbb{Q} / \mathbb{Z}$. The endomorphism $\rho_{n}$ associated to an element $n \in S$ of the (multiplicative) semigroup $S=\mathbb{N}=\mathbb{Z}_{>0}$ is given on the canonical basis $e_{r} \in \mathbb{Q}[\mathbb{Q} / \mathbb{Z}], r \in \mathbb{Q} / \mathbb{Z}$, by

$$
\rho_{n}\left(e_{r}\right)=\frac{1}{n} \sum_{n s=r} e_{s}
$$

The corresponding analytic endomotive is the crossed product $C^{*}$-algebra

$$
\mathcal{A}=C^{*}(\mathbb{Q} / \mathbb{Z}) \rtimes \mathbb{N} .
$$

The Galois action is given by composing a character $\chi: A_{n} \rightarrow \overline{\mathbb{Q}}$ with an element $g$ of the Galois group $G=\operatorname{Gal}(\overline{\mathbb{Q}} / \mathbb{Q})$. Since $\chi$ is determined by the $n$-th root of unity $\chi\left(e_{1 / n}\right)$, one obtains the cyclotomic action.

In the case of the Bost-Connes endomotive, the state $\varphi$ on $\mathcal{A}$ induced by the measure $\mu$ on $X=\hat{\mathbb{Z}}$ is of the form

$$
\varphi(f)=\int_{\hat{\mathbb{Z}}} f(1, \rho) d \mu(\rho),
$$


and the modular automorphism group restricts to the $C^{*}$-algebra as the time evolution of the BC system, cf. [5], [11] and $\S 4$ of [13].

The dual system of the Bost-Connes system is best described in terms of commensurability classes of $\mathbb{Q}$-lattices. In [12 the Bost-Connes system is reinterpreted as the noncommutative space describing the relation of commensurability for 1-dimensional $\mathbb{Q}$-lattices up to scaling. One can also consider the same equivalence relation without dividing out by the scaling action. If we let $\mathcal{G}_{1}$ denote the groupoid of the commensurability relation on 1-dimensional $\mathbb{Q}$-lattices and $\mathcal{G}_{1} / \mathbb{R}_{+}^{*}$ the one obtained after moding out by scaling, we identify the $C^{*}$-algebra of the Bost-Connes system with $C^{*}\left(\mathcal{G}_{1} / \mathbb{R}_{+}^{*}\right)(c f$. [12]). The algebra $\hat{\mathcal{A}}$ of the dual system is then obtained in the following way ( $c f$. [11]). There is a $C^{*}$-algebra isomorphism $\iota: \hat{\mathcal{A}} \rightarrow C^{*}\left(\mathcal{G}_{1}\right)$ of the form

$$
\iota(X)(k, \rho, \lambda)=\int_{\mathbb{R}} x(t)(k, \rho) \lambda^{i t} d t
$$

for $(k, \rho, \lambda) \in \mathcal{G}_{1}$ and $X=\int x(t) U_{t} d t \in \hat{\mathcal{A}}$.

3.2. Scaling as Frobenius in characteristic zero. In the general setting described in [11] one denotes by $D(\mathcal{A}, \varphi)$ the cokernel of the morphism (3.5), viewed as a module in the cyclic category. The notation is meant to recall the dependence of the construction on the initial data of an analytic endomotive $\mathcal{A}$ and a state $\varphi$. The cyclic module $D(\mathcal{A}, \varphi)$ inherits a scaling action of $\mathbb{R}_{+}^{*}$ and one can consider the induced action on the cyclic homology $H C_{0}(D(\mathcal{A}, \varphi))$. We argued in [11] that this cyclic homology with the induced scaling action plays a role analogous to the role played by the Frobenius action on étale cohomology in the algebro-geometric context. Our main supporting evidence is the Lefschetz trace formula for this action that gives a cohomological interpretation of the spectral realization of the zeros of the Riemann zeta function of [10]. We return to discuss the Lefschetz trace formula for the more general case of global fields in $\$ 6$ below.

The main results of [10] show that we have the following setup. There is an exact sequence of Hilbert spaces

$$
0 \rightarrow L_{\delta}^{2}\left(\mathbb{A}_{\mathbb{Q}} / \mathbb{Q}^{*}\right)_{0} \rightarrow L_{\delta}^{2}\left(\mathbb{A}_{\mathbb{Q}} / \mathbb{Q}^{*}\right) \rightarrow \mathbb{C}^{2} \rightarrow 0
$$

which defines the subspace $L_{\delta}^{2}\left(\mathbb{A}_{\mathbb{Q}} / \mathbb{Q}^{*}\right)_{0}$ by imposing the conditions $f(0)=0$ and $\hat{f}(0)=0$ and a suitable decay condition imposed by the weight $\delta$. The space $L_{\delta}^{2}\left(\mathbb{A}_{\mathbb{Q}} / \mathbb{Q}^{*}\right)_{0}$ fits into another exact sequence of Hilbert spaces of the form

$$
0 \rightarrow L_{\delta}^{2}\left(\mathbb{A}_{\mathbb{Q}} / \mathbb{Q}^{*}\right)_{0} \stackrel{\mathfrak{E}}{\rightarrow} L_{\delta}^{2}\left(C_{\mathbb{Q}}\right) \rightarrow \mathcal{H} \rightarrow 0
$$

where the map $\mathfrak{E}$ is defined by

$$
\mathfrak{E}(f)(g)=|g|^{1 / 2} \sum_{q \in \mathbb{Q}^{*}} f(q g), \quad \forall g \in C_{\mathbb{Q}}=\mathbb{A}_{\mathbb{Q}}^{*} / \mathbb{Q}^{*} .
$$

The map is equivariant with respect to the actions of $C_{\mathbb{Q}}$ i.e.

$$
\mathfrak{E} \circ \vartheta_{a}(\gamma)=|\gamma|^{1 / 2} \vartheta_{m}(\gamma) \circ \mathfrak{E}
$$


where $\left(\vartheta_{a}(\gamma) \xi\right)(x)=\xi\left(\gamma^{-1} x\right)$ for $\xi \in L_{\delta}^{2}\left(\mathbb{A}_{\mathbb{Q}} / \mathbb{Q}^{*}\right)_{0}$ and similarly $\vartheta_{m}(\gamma)$ is the regular representation of $C_{\mathbb{K}}$.

We showed in [1] that the map $\mathfrak{E}$, translated from the context of Hilbert spaces to that of nuclear spaces as in [32, has a natural interpretation in terms of the "cooling and distillation process" for the $\mathrm{BC}$ endomotive. In fact, we showed in [11] that, if $(\mathcal{A}, \sigma)$ denotes the $\mathrm{BC}$ system, then the following properties hold.

(1) For $\beta>1$ there is a canonical isomorphism

$$
\tilde{\Omega}_{\beta} \simeq \hat{\mathbb{Z}}^{*} \times \mathbb{R}_{+}^{*} \simeq C_{\mathbb{Q}}
$$

of $\tilde{\Omega}_{\beta}$ with the space of invertible 1-dimensional $\mathbb{Q}$-lattices.

(2) For $X \in \hat{\mathcal{A}}$ and $f=\iota(X) \in C^{*}\left(\mathcal{G}_{1}\right)$, the cooling map (3.5) takes the form

$$
\delta(X)(u, \lambda)=\sum_{n \in \mathbb{N}=\mathbb{Z}>0} f(1, n u, n \lambda), \quad \forall(u, \lambda) \in C_{\mathbb{Q}} \simeq \tilde{\Omega}_{\beta} .
$$

One can compare directly the right hand side of (3.14) with the map $\mathfrak{E}$ (up to the normalization by $|j|^{1 / 2}$ ) written as in (3.11) by considering a function $f(\rho, v)=f(1, \rho, v)$ and its unique extension $\tilde{f}$ to adeles where $f$ is extended by 0 outside $\hat{\mathbb{Z}} \times \mathbb{R}^{*}$ and one requires the parity

$$
\tilde{f}(-u,-\lambda)=f(u, \lambda) .
$$

This gives then

$$
\sum_{n \in \mathbb{N}} f(1, n u, n \lambda)=\frac{1}{2} \sum_{q \in \mathbb{Q}^{*}} \tilde{f}(q j), \quad \text { where } j=(u, \lambda) \in C_{\mathbb{Q}} .
$$

\section{The Adeles Class SPACE}

Let $\mathbb{K}$ be a global field, with $\mathbb{A}_{\mathbb{K}}$ its ring of adeles.

Definition 4.1. The adeles class space of a global field $\mathbb{K}$ is the quotient $\mathbb{A}_{\mathbb{K}} / \mathbb{K}^{*}$.

When viewed from the classical standpoint this is a "bad quotient" due to the ergodic nature of the action which makes the quotient ill behaved topologically. Thus, following the general philosophy of noncommutative geometry, we describe it by a noncommutative algebra of coordinates, which allows one to continue to treat the quotient as a "nice quotient" in the context of noncommutative geometry.

A natural choice of the algebra is the cross product

$$
C_{0}\left(\mathbb{A}_{\mathbb{K}}\right) \rtimes \mathbb{K}^{*} \quad \text { with the smooth subalgebra } \mathcal{S}\left(\mathbb{A}_{\mathbb{K}}\right) \rtimes \mathbb{K}^{*} .
$$

A better description can be given in terms of groupoids.

Consider the groupoid law $\mathcal{G}_{\mathbb{K}}=\mathbb{K}^{*} \ltimes \mathbb{A}_{\mathbb{K}}$ given by

$$
(k, x) \circ\left(k^{\prime}, y\right)=\left(k k^{\prime}, y\right), \quad \forall k, k^{\prime} \in \mathbb{K}^{*}, \quad \text { and } \forall x, y \in \mathbb{A}_{\mathbb{K}} \text { with } x=k^{\prime} y,
$$

with the composition (4.2) defined whenever the source $s(k, x)=x$ agrees with the range $r\left(k^{\prime}, y\right)=k^{\prime} y$. 
Lemma 4.2. The algebras (4.1) are, respectively, the groupoid $C^{*}$-algebra $C^{*}\left(\mathcal{G}_{\mathbb{K}}\right)$ and its dense subalgebra $\mathcal{S}\left(\mathcal{G}_{\mathbb{K}}\right)$.

Proof. The product in the groupoid algebra is given by the associative convolution product

$$
\left(f_{1} * f_{2}\right)(k, x)=\sum_{s \in \mathbb{K}^{*}} f_{1}\left(k s^{-1}, s x\right) f_{2}(s, x),
$$

and the adjoint is given by $f^{*}(k, x)=\overline{f\left(k^{-1}, k x\right)}$.

The functions (on the groupoid) associated to $f \in \mathcal{S}\left(\mathbb{A}_{K}\right)$ and $U_{k}$ are given, respectively, by

$$
\begin{array}{llll}
f(1, x)=f(x) & \text { and } & f(k, x)=0 & \forall k \neq 1 \\
U_{k}(k, x)=1 & \text { and } & U_{g}(k, x)=0 & \forall g \neq k .
\end{array}
$$

The product $f U_{k}$ is then the convolution product of the groupoid.

The algebra $\mathcal{S}\left(\mathcal{G}_{\mathbb{K}}\right)$ is obtained by considering finite sums of the form

$$
\sum_{k \in \mathbb{K}^{*}} f_{k} U_{k}, \quad \text { for } f_{k} \in \mathcal{S}\left(\mathbb{A}_{\mathbb{K}}\right) .
$$

The product is given by the convolution product

$$
\left(U_{k} f U_{k}^{*}\right)(x)=f\left(k^{-1} x\right),
$$

for $f \in \mathcal{S}\left(\mathbb{A}_{\mathbb{K}}\right), k \in \mathbb{K}^{*}$, and $x \in \mathbb{A}_{\mathbb{K}}$.

4.1. Cyclic module. We can associate to the algebra $\mathcal{S}\left(\mathcal{G}_{\mathbb{K}}\right)$ of the adeles class space an object in the category of $\Lambda$-modules. This means that we consider the cyclic module $\mathcal{S}\left(\mathcal{G}_{\mathbb{K}}\right)^{\natural}$ and the two cyclic morphisms

$$
\varepsilon_{j}: \mathcal{S}\left(\mathcal{G}_{\mathbb{K}}\right)^{\natural} \rightarrow \mathbb{C}
$$

given by

$$
\varepsilon_{0}\left(\sum f_{k} U_{k}\right)=f_{1}(0) \quad \text { and } \quad \varepsilon_{1}\left(\sum f_{k} U_{k}\right)=\int_{\mathbb{A}_{\mathbb{K}}} f_{1}(x) d x
$$

and in higher degree by

$$
\varepsilon_{j}^{\natural}\left(a^{0} \otimes \cdots \otimes a^{n}\right)=\varepsilon_{j}\left(a^{0} \cdots a^{n}\right) .
$$

The morphism $\varepsilon_{1}$ is given by integration on $\mathbb{A}_{\mathbb{K}}$ with respect to the additive Haar measure. This is $\mathbb{K}^{*}$ invariant, hence it defines a trace on $\mathcal{S}\left(\mathcal{G}_{\mathbb{K}}\right)$. In the case of $\mathbb{K}=\mathbb{Q}$, this corresponds to the dual trace $\tau_{\varphi}$ for the $\mathrm{KMS}_{1}$-state $\varphi$ associated to the time evolution of the $\mathrm{BC}$ system. The morphism $\varepsilon_{0}$ here takes into account the fact that we are imposing a vanishing condition at $0 \in \mathbb{A}_{\mathbb{K}}(c f$. [11] and [13] Chapter 4$)$. In fact, the $\Lambda$-module we associate to $\mathcal{S}\left(\mathcal{G}_{\mathbb{K}}\right)$ is given by

$$
\mathcal{S}\left(\mathcal{G}_{\mathbb{K}}\right)_{0}^{\natural}:=\operatorname{Ker} \varepsilon_{0}^{\natural} \cap \operatorname{Ker} \varepsilon_{1}^{\natural} .
$$

Note that since $\mathcal{S}\left(\mathcal{G}_{\mathbb{K}}\right)$ is non-unital, the cyclic module $\mathcal{S}\left(\mathcal{G}_{\mathbb{K}}\right)^{\natural}$ is obtained using the adjunction of a unit to $\mathcal{S}\left(\mathcal{G}_{\mathbb{K}}\right)$. 
4.2. The restriction map. Consider the ideles $\mathbb{A}_{\mathbb{K}}^{*}=G_{1}\left(\mathbb{A}_{\mathbb{K}}\right)$ of $\mathbb{K}$ with their natural locally compact topology induced by the map

$$
\mathbb{A}_{\mathbb{K}}^{*} \ni g \mapsto\left(g, g^{-1}\right) \text {. }
$$

We can see the ideles class group $C_{\mathbb{K}}=\mathbb{A}_{\mathbb{K}}^{*} / \mathbb{K}^{*}$ as a subspace of the adeles class space $X_{\mathbb{K}}=\mathbb{A}_{\mathbb{K}} / \mathbb{K}^{*}$ in the following way.

Lemma 4.3. The pairs $\left((k, x),\left(k^{\prime}, y\right)\right) \in \mathcal{G}_{\mathbb{K}}$ such that both $x$ and $y$ are in $\mathbb{A}_{\mathbb{K}}^{*}$ form a full subgroupoid of $\mathcal{G}_{\mathbb{K}}$ which is isomorphic to $\mathbb{K}^{*} \ltimes \mathbb{A}_{\mathbb{K}}^{*}$.

Proof. Elements of $\mathbb{A}_{\mathbb{K}}$ whose orbit under the $\mathbb{K}^{*}$ action contains an idele are also ideles. Thus, we obtain a groupoid that is a full subcategory of $\mathcal{G}_{\mathbb{K}}$.

This implies the existence of a restriction map. Consider the map

$$
\rho:\left.\mathcal{S}\left(\mathbb{A}_{K}\right) \ni f \mapsto f\right|_{\mathbb{A}_{\mathbb{K}}^{*}}
$$

We denote by $C_{\rho}\left(\mathbb{A}_{\mathbb{K}}^{*}\right) \subset C\left(\mathbb{A}_{\mathbb{K}}^{*}\right)$ the range of $\rho$.

Corollary 4.4. The restriction map $\rho$ of (4.12) extends to an algebra homomorphism

$$
\rho: \mathcal{S}\left(\mathcal{G}_{\mathbb{K}}\right) \rightarrow C_{\rho}\left(\mathbb{A}_{\mathbb{K}}^{*}\right) \rtimes \mathbb{K}^{*}
$$

Proof. The map (4.12) induced by the inclusion $\mathbb{A}_{\mathbb{K}}^{*} \subset \mathbb{A}_{\mathbb{K}}$ is continuous and $\mathbb{K}^{*}$ equivariant hence the map

$$
\rho\left(\sum_{k \in \mathbb{K}^{*}} f_{k} U_{k}\right)=\sum_{k \in \mathbb{K}^{*}} \rho\left(f_{k}\right) U_{k}
$$

is an algebra homomorphism.

The action of $\mathbb{K}^{*}$ on $\mathbb{A}_{\mathbb{K}}^{*}$ is free and proper so that we have an equivalence of the locally compact groupoids $\mathbb{K}^{*} \ltimes \mathbb{A}_{\mathbb{K}}^{*}$ and $\mathbb{A}_{\mathbb{K}}^{*} / \mathbb{K}^{*}=C_{\mathbb{K}}$. We use the exact sequence of locally compact groups

$$
1 \rightarrow \mathbb{K}^{*} \rightarrow \mathbb{A}_{\mathbb{K}}^{*} \stackrel{p}{\rightarrow} C_{\mathbb{K}} \rightarrow 1
$$

to parameterize the orbits of $\mathbb{K}^{*}$ as the fibers $p^{-1}(x)$ for $x \in C_{\mathbb{K}}$. By construction the Hilbert spaces

$$
\mathcal{H}_{x}=\ell^{2}\left(p^{-1}(x)\right), \quad \forall x \in C_{K}
$$

form a continuous field of Hilbert spaces over $C_{\mathbb{K}}$. We let $\mathcal{L}^{1}\left(\mathcal{H}_{x}\right)$ be the Banach algebra of trace class operators in $\mathcal{H}_{x}$, these form a continuous field over $C_{\mathbb{K}}$.

Proposition 4.5. The restriction map $\rho$ of (4.12) extends to an algebra homomorphism

$$
\rho: \mathcal{S}\left(\mathcal{G}_{\mathbb{K}}\right) \rightarrow C\left(C_{\mathbb{K}}, \mathcal{L}^{1}\left(\mathcal{H}_{x}\right)\right) .
$$

Proof. Each $p^{-1}(x)$ is globally invariant under the action of $\mathbb{K}^{*}$ so the crossed product rules in $C_{\rho}\left(\mathbb{A}_{\mathbb{K}}^{*}\right) \rtimes \mathbb{K}^{*}$ are just multiplication of operators in $\mathcal{H}_{x}$. To show that the obtained operators are in $\mathcal{L}^{1}$ we just need to consider monomials $f_{k} U_{k}$. In that case the only non-zero matrix elements correspond to $k=x y^{-1}$. It is enough to show that, for any $f \in \mathcal{S}\left(\mathbb{A}_{\mathbb{K}}\right)$, the function $k \mapsto f(k b)$ is summable. This 
follows from the discreteness of $b \mathbb{K} \subset \mathbb{A}_{\mathbb{K}}$ and the construction of the BruhatSchwartz space $\mathcal{S}\left(\mathbb{A}_{\mathbb{K}}\right)$, cf. [10]. In fact the associated operator is of finite rank when $f$ has compact support. In general what happens is that the sum will look like the sum over $\mathbb{Z}$ of the values $f(n b)$ of a Schwartz function $f$ on $\mathbb{R}$.

In general the exact sequence (4.14) does not split and one does not have a natural $C_{\mathbb{K}}$-equivariant trivialization of the continuous field $\mathcal{H}_{x}$. Thus it is important in the general case to keep the nuance between the algebras $C\left(C_{\mathbb{K}}, \mathcal{L}^{1}\left(\mathcal{H}_{x}\right)\right)$ and $C\left(C_{\mathbb{K}}\right)$. We shall first deal with the special case $\mathbb{K}=\mathbb{Q}$ in which this issue does not arise.

4.3. The Morita equivalence and cokernel for $\mathbb{K}=\mathbb{Q}$. The exact sequence (4.14) splits for $\mathbb{K}=\mathbb{Q}$ and admits a natural continuous section which corresponds to the open and closed fundamental domain $\Delta_{\mathbb{Q}}=\hat{\mathbb{Z}}^{*} \times \mathbb{R}_{+}^{*} \subset \mathbb{A}_{\mathbb{Q}}^{*}$ for the action of $\mathbb{Q}^{*}$ on ideles. This allows us to construct a cyclic morphism between the cyclic modules associated, respectively, to the algebra $C_{\rho}\left(\mathbb{A}_{\mathbb{Q}}^{*}\right) \rtimes \mathbb{Q}^{*}$ and to a suitable algebra $C_{\rho}\left(C_{\mathbb{Q}}\right)$ of functions on $C_{\mathbb{Q}}$.

Lemma 4.6. The composition $d_{\mathbb{Q}} \circ e_{\mathbb{Q}}$ of the maps

$$
e_{\mathbb{Q}}:(k, h b) \mapsto(b,(k, h)), \quad \text { and } \quad d_{\mathbb{Q}}(k, h)=(k h, h)
$$

with $b \in \Delta_{\mathbb{Q}}$ and $k, h \in \mathbb{Q}^{*}$, gives an isomorphism of the locally compact groupoids

$$
\mathbb{Q}^{*} \ltimes \mathbb{A}_{\mathbb{Q}}^{*} \simeq \Delta_{\mathbb{Q}} \times \mathbb{Q}^{*} \times \mathbb{Q}^{*}
$$

Proof. The map $e_{\mathbb{Q}}$ realizes an isomorphism between the locally compact groupoids

$$
\mathbb{Q}^{*} \ltimes \mathbb{A}_{\mathbb{Q}}^{*} \simeq \Delta_{\mathbb{Q}} \times\left(\mathbb{Q}^{*} \ltimes \mathbb{Q}^{*}\right),
$$

where $\mathbb{Q}^{*} \ltimes \mathbb{Q}^{*}$ is the groupoid of the action of $\mathbb{Q}^{*}$ on itself by multiplication. The latter is isomorphic to the trivial groupoid $\mathbb{Q}^{*} \times \mathbb{Q}^{*}$ via the map $d_{\mathbb{Q}}$.

We then have the following result.

Proposition 4.7. The map

$$
\sum_{k \in \mathbb{Q}^{*}} f_{k} U_{k} \mapsto M_{b}(x, y)=f_{x y^{-1}}(x b),
$$

for $x, y \in \mathbb{Q}^{*}$ with $k=x y^{-1}$ and $b \in \Delta_{\mathbb{Q}}$, defines an algebra homomorphism

$$
C_{\rho}\left(\mathbb{A}_{\mathbb{Q}}^{*}\right) \rtimes \mathbb{Q}^{*} \rightarrow C\left(\Delta_{\mathbb{Q}}, M_{\infty}(\mathbb{C})\right)
$$

to the algebra of matrix valued functions on $\Delta_{\mathbb{Q}}$. For any $f \in \mathcal{S}\left(\mathcal{G}_{\mathbb{Q}}\right)$ the element $M_{b}$ obtained in this way is of trace class.

Proof. We use the groupoid isomorphism (4.17) to write $k=x y^{-1}$ and $k h b=x b$, for $x=k h$ and $y=h$. The second statement follows from Proposition 4.5 .

Let $\pi=M \circ \rho: \mathcal{S}\left(\mathcal{G}_{\mathbb{K}}\right) \rightarrow C\left(\Delta_{\mathbb{Q}}, M_{\infty}(\mathbb{C})\right)$ be the composition of the restriction map $\rho$ of (4.13) with the algebra morphism (4.19). Since the trace $\operatorname{Tr}$ on $M_{\infty}(\mathbb{C})$ 
gives a cyclic morphism one can use this to obtain a morphism of cyclic modules $(\operatorname{Tr} \circ \pi)^{\natural}$, which we now describe explicitly. We let, in the number field case,

$$
\mathbf{S}\left(C_{\mathbb{K}}\right)=\cap_{\beta \in \mathbb{R}} \mu^{\beta} \mathcal{S}\left(C_{\mathbb{K}}\right),
$$

where $\mu \in C\left(C_{\mathbb{K}}\right)$ is the module morphism from $C_{\mathbb{K}}$ to $\mathbb{R}_{+}^{*}$. In the function field case one can simply use for $\mathbf{S}\left(C_{\mathbb{K}}\right)$ the Schwartz functions with compact support.

Proposition 4.8. The map $\operatorname{Tr} \circ \pi$ defines a morphism $(\operatorname{Tr} \circ \pi)^{\natural}$ of cyclic modules from $\mathcal{S}\left(\mathcal{G}_{\mathbb{Q}}\right)_{0}^{\natural}$ to the cyclic submodule $\mathbf{S}^{\natural}\left(C_{\mathbb{Q}}\right) \subset C\left(C_{\mathbb{Q}}\right)^{\natural}$ whose elements are continuous functions whose restriction to the main diagonal belongs to $\mathbf{S}\left(C_{\mathbb{Q}}\right)$.

Proof. By Proposition 4.7 the map $\pi$ is an algebra homomorphism from $\mathcal{S}\left(\mathcal{G}_{\mathbb{Q}}\right)$ to $C\left(\Delta_{\mathbb{Q}}, \mathcal{L}^{1}\right) \sim C\left(C_{\mathbb{Q}}, \mathcal{L}^{1}\right)$. We need to show that the corresponding cyclic morphism using $\operatorname{Tr}^{\natural}$ lands in the cyclic submodule $S^{\natural}\left(C_{\mathbb{Q}}\right)$.

For simplicity we can just restrict to the case of monomials, where we consider elements of the form

$$
Z=f_{k_{0}} U_{k_{0}} \otimes f_{k_{1}} U_{k_{1}} \otimes \cdots \otimes f_{k_{n}} U_{k_{n}} .
$$

The matrix valued functions associated to the monomials $f_{k_{j}} U_{k_{j}}$ as in Proposition 4.7 have matrix elements at a point $b \in \Delta_{\mathbb{Q}}$ that are non zero only for $x_{j+1}=$ $x_{j} k_{j}^{-1}$ and are of the form

$$
f_{k_{j}} U_{k_{j}} \mapsto M_{b}\left(x_{j}, x_{j+1}\right)=f_{k_{j}}\left(x_{j} b\right) .
$$

Composing with the cyclic morphism $\operatorname{Tr}^{\natural}$ gives

$$
(\operatorname{Tr} \circ \pi)^{\natural}(Z)\left(b_{0}, b_{1}, \ldots, b_{n}\right)=\sum \prod M_{b_{j}}\left(x_{j}, x_{j+1}\right)
$$

where the $x_{j} \in \mathbb{K}^{*}$ and $x_{n+1}=x_{0}$. Let $\gamma_{0}=1$ and $\gamma_{j+1}=k_{j} \gamma_{j}$. Then we find that $(\operatorname{Tr} \circ \pi)^{\natural}(Z)=0$, unless $\prod_{j} k_{j}=1$, i.e. $\gamma_{n+1}=1$. In this case we obtain

$$
\operatorname{Tr} \circ \pi(Z)\left(b_{0}, b_{1}, \cdots, b_{n}\right)=\sum_{k \in \mathbb{Q}^{*}} \prod_{j=0}^{n} f_{k_{j}}\left(\gamma_{j}^{-1} k b_{j}\right), \quad \forall b_{j} \in \Delta_{\mathbb{Q}} .
$$

For $n=0$ the formula (4.24) reduces to

$$
\operatorname{Tr} \circ \pi(f)(b)=\sum_{k \in \mathbb{Q}^{*}} f(k b), \quad \forall b \in \Delta_{\mathbb{Q}}, \quad \forall f \in \mathcal{S}\left(\mathbb{A}_{\mathbb{Q}}\right)_{0},
$$

where $\mathcal{S}\left(\mathbb{A}_{\mathbb{Q}}\right)_{0}=\operatorname{Ker} \varepsilon_{0} \cap \operatorname{Ker} \varepsilon_{1} \subset \mathcal{S}\left(\mathbb{A}_{\mathbb{Q}}\right)$. This gives an element of $\mathbf{S}\left(C_{\mathbb{Q}}\right)$, by Lemma 2 Appendix 1 of [10]. In general, (4.24) gives a continuous function of $n+1$ variables on $C_{\mathbb{Q}}$, and its restriction to the main diagonal belongs to $\mathbf{S}\left(C_{\mathbb{Q}}\right)$.

Since the category of cyclic modules is an abelian category, we can consider the cokernel in the category of $\Lambda$-modules of the cyclic morphism $(\operatorname{Tr} \circ \pi)^{\natural}$, with $\pi$ the composite of (4.13) and (4.19). This works nicely for $\mathbb{K}=\mathbb{Q}$ but makes use of the splitting of the exact sequence (4.14). 
4.4. The cokernel of $\rho$ for general global fields. To handle the general case in a canonical manner one just needs to work directly with $C\left(C_{\mathbb{K}}, \mathcal{L}^{1}\left(\mathcal{H}_{x}\right)\right)$ instead of $C\left(C_{\mathbb{K}}\right)$ and express at that level the decay condition of the restrictions to the diagonal in the cyclic submodule $\mathbf{S}^{\natural}\left(C_{\mathbb{Q}}\right)$ of Proposition 4.8.

Definition 4.9. We define $\mathbf{S}^{\natural}\left(C_{\mathbb{K}}, \mathcal{L}^{1}\left(\mathcal{H}_{x}\right)\right)$ to be the cyclic submodule of the cyclic module $C\left(C_{\mathbb{K}}, \mathcal{L}^{1}\left(\mathcal{H}_{x}\right)\right)^{\natural}$, whose elements are continuous functions such that the trace of the restriction to the main diagonal belongs to $\mathbf{S}\left(C_{\mathbb{K}}\right)$.

Note that for $T \in C\left(C_{\mathbb{K}}, \mathcal{L}^{1}\left(\mathcal{H}_{x}\right)\right)^{\natural}$ of degree $n, T\left(x_{0}, \ldots, x_{n}\right)$ is an operator in $\mathcal{H}_{x_{0}} \otimes \ldots \otimes \mathcal{H}_{x_{n}}$. On the diagonal, $x_{j}=x$ for all $j$, the trace map corresponding to $\operatorname{Tr}^{\natural}$ is given by

$$
\operatorname{Tr}^{\natural}\left(T_{0} \otimes T_{1} \otimes \ldots \otimes T_{n}\right)=\operatorname{Tr}\left(T_{0} T_{1} \ldots T_{n}\right) .
$$

This makes sense since on the diagonal all the Hilbert spaces $\mathcal{H}_{x_{j}}$ are the same. The argument of Proposition 4.8 extends to the general case and shows that the cyclic morphism $\rho^{\natural}$ of the restriction map $\rho$ lands in $\mathbf{S}^{\natural}\left(C_{\mathbb{K}}, \mathcal{L}^{1}\left(\mathcal{H}_{x}\right)\right)$.

Definition 4.10. We define $\mathcal{H}^{1}\left(\mathbb{A}_{\mathbb{K}} / \mathbb{K}^{*}, C_{\mathbb{K}}\right)$ to be the cokernel of the cyclic morphism

$$
\rho^{\natural}: \mathcal{S}\left(\mathcal{G}_{\mathbb{K}}\right)_{0}^{\natural} \rightarrow \mathbf{S}^{\natural}\left(C_{\mathbb{K}}, \mathcal{L}^{1}\left(\mathcal{H}_{x}\right)\right)
$$

Moreover, an important issue arises, since the ranges of continuous linear maps are not necessarily closed subspaces. In order to preserve the duality between cyclic homology and cyclic cohomology we shall define the cokernel of a cyclic map $T: \mathcal{A}^{\natural} \rightarrow \mathcal{B}^{\natural}$ as the quotient of $\mathcal{B}^{\natural}$ by the closure of the range of $T$. In a dual manner, the kernel of the transposed map $T^{t}: \mathcal{B}^{\sharp} \rightarrow \mathcal{A}^{\sharp}$ is automatically closed and is the dual of the above.

The choice of the notation $\mathcal{H}^{1}\left(\mathbb{A}_{\mathbb{K}} / \mathbb{K}^{*}, C_{\mathbb{K}}\right)$ is explained by the fact that we consider this a first cohomology group, in the sense that it is a cokernel in a sequence of cyclic homology groups for the inclusion of the ideles class group in the adeles class space (dually for the restriction map of algebras), hence we can think of it as giving rise to an $H^{1}$ in the relative cohomology sequence of an inclusion of $C_{\mathbb{K}}$ in the noncommutative space $\mathbb{A}_{\mathbb{K}} / \mathbb{K}^{*}$. We can use the result of [7], describing the cyclic (co)homology in terms of derived functors in the category of cylic modules, to write the cyclic homology as

$$
H C_{n}(\mathcal{A})=\operatorname{Tor}_{n}\left(\mathbb{C}^{\natural}, \mathcal{A}^{\natural}\right) .
$$

Thus, we obtain a cohomological realization of the cyclic module $\mathcal{H}^{1}\left(\mathbb{A}_{\mathbb{K}} / \mathbb{K}^{*}, C_{\mathbb{K}}\right)$ by setting

$$
H^{1}\left(\mathbb{A}_{\mathbb{K}} / \mathbb{K}^{*}, C_{\mathbb{K}}\right):=\operatorname{Tor}\left(\mathbb{C}^{\natural}, \mathcal{H}^{1}\left(\mathbb{A}_{\mathbb{K}} / \mathbb{K}^{*}, C_{\mathbb{K}}\right)\right) .
$$

We think of this as an $H^{1}$ because of its role as a relative term in a cohomology exact sequence of the pair $\left(\mathbb{A}_{\mathbb{K}} / \mathbb{K}^{*}, C_{\mathbb{K}}\right)$.

We now show that $H^{1}\left(\mathbb{A}_{\mathbb{K}} / \mathbb{K}^{*}, C_{\mathbb{K}}\right)$ carries an action of $C_{\mathbb{K}}$, which we can view as the abelianization $W_{\mathbb{K}}^{a b} \sim C_{\mathbb{K}}$ of the Weil group. This action is induced by the multiplicative action of $C_{\mathbb{K}}$ on $\mathbb{A}_{\mathbb{K}} / \mathbb{K}^{*}$ and on itself. This generalizes to 
global fields the action of $C_{\mathbb{Q}}=\hat{\mathbb{Z}}^{*} \times \mathbb{R}_{+}^{*}$ on $H C_{0}(D(\mathcal{A}, \varphi))$ for the Bost-Connes endomotive ( $c f$. [11]).

Proposition 4.11. The cyclic modules $\mathcal{S}\left(\mathcal{G}_{\mathbb{K}}\right)_{0}^{\natural}$ and $\mathbf{S}^{\natural}\left(C_{\mathbb{K}}, \mathcal{L}^{1}\left(\mathcal{H}_{x}\right)\right)$ are endowed with an action of $\mathbb{A}_{\mathbb{K}}^{*}$ and the morphism $\rho^{\natural}$ is $\mathbb{A}_{\mathbb{K}}^{*}$-equivariant. This induces an action of $C_{\mathbb{K}}$ on $H^{1}\left(\mathbb{A}_{\mathbb{K}} / \mathbb{K}^{*}, C_{\mathbb{K}}\right)$.

Proof. For $\gamma \in \mathbb{A}_{\mathbb{K}}^{*}$ one defines an action by automorphisms of the algebra $\mathcal{A}=$ $\mathcal{S}\left(\mathcal{G}_{\mathbb{K}}\right)$ by setting

$$
\begin{gathered}
\vartheta_{a}(\gamma)(f)(x):=f\left(\gamma^{-1} x\right), \quad \text { for } f \in \mathcal{S}\left(\mathbb{A}_{\mathbb{K}}\right), \\
\vartheta_{a}(\gamma)\left(\sum_{k \in \mathbb{K}^{*}} f_{k} U_{k}\right):=\sum_{k \in \mathbb{K}^{*}} \vartheta_{a}(\gamma)\left(f_{k}\right) U_{k} .
\end{gathered}
$$

This action is inner for $\gamma \in \mathbb{K}^{*}$ and induces an outer action

$$
C_{\mathbb{K}} \rightarrow \operatorname{Out}\left(\mathcal{S}\left(\mathcal{G}_{\mathbb{K}}\right)\right) .
$$

Similarly, the continuous field $\mathcal{H}_{x}=\ell^{2}\left(p^{-1}(x)\right)$ over $C_{\mathbb{K}}$ is $\mathbb{A}_{\mathbb{K}}^{*}$-equivariant for the action of $\mathbb{A}_{\mathbb{K}}^{*}$ on $C_{\mathbb{K}}$ by translations, and the equality

$$
(V(\gamma) \xi)(y):=\xi\left(\gamma^{-1} y\right), \quad \forall y \in p^{-1}(\gamma x), \xi \in \ell^{2}\left(p^{-1}(x)\right)
$$

defines an isomorphism $\mathcal{H}_{x} \stackrel{V(\gamma)}{\longrightarrow} \mathcal{H}_{\gamma x}$. One obtains then an action of $\mathbb{A}_{\mathbb{K}}^{*}$ on $C\left(C_{\mathbb{K}}, \mathcal{L}^{1}\left(\mathcal{H}_{x}\right)\right)$ by setting

$$
\vartheta_{m}(\gamma)(f)(x):=V(\gamma) f\left(\gamma^{-1} x\right) V\left(\gamma^{-1}\right), \quad \forall f \in C\left(C_{\mathbb{K}}, \mathcal{L}^{1}\left(\mathcal{H}_{x}\right)\right) .
$$

The morphism $\rho$ is $\mathbb{A}_{\mathbb{K}^{-}}^{*}$-equivariant, so that one obtains an induced action on the cokernel $\mathcal{H}^{1}\left(\mathbb{A}_{\mathbb{K}} / \mathbb{K}^{*}, C_{\mathbb{K}}\right)$. This action is inner for $\gamma \in \mathbb{K}^{*}$ and thus induces an action of $C_{\mathbb{K}}$ on $H^{1}\left(\mathbb{A}_{\mathbb{K}} / \mathbb{K}^{*}, C_{\mathbb{K}}\right)$.

We denote by

$$
C_{\mathbb{K}} \ni \gamma \mapsto \underline{\vartheta}_{m}(\gamma)
$$

the induced action on $H^{1}\left(\mathbb{A}_{\mathbb{K}} / \mathbb{K}^{*}, C_{\mathbb{K}}\right)$.

We have a non-canonical isomorphism

$$
C_{\mathbb{K}} \simeq C_{\mathbb{K}, 1} \times N
$$

where $N \subset \mathbb{R}_{+}^{*}$ is the range of the norm $|\cdot|: C_{\mathbb{K}} \rightarrow \mathbb{R}_{+}^{*}$. For number fields this is $N=\mathbb{R}_{+}^{*}$, while for function fields in positive characteristic $N \simeq \mathbb{Z}$ is the subgroup $q^{\mathbb{Z}} \subset \mathbb{R}_{+}^{*}$ with $q=p^{\ell}$ the cardinality of the field of constants. We denote by $\widehat{C_{\mathbb{K}, 1}}$ the group of characters of the compact subgroup $C_{\mathbb{K}, 1} \subset C_{\mathbb{K}}$, i.e. the Pontrjagin dual of $C_{\mathbb{K}, 1}$. Given a character $\chi$ of $C_{\mathbb{K}, 1}$, we let $\tilde{\chi}$ denote the unique extension of $\chi$ to $C_{\mathbb{K}}$ which is equal to one on $N$.

One obtains a decomposition of $H^{1}\left(\mathbb{A}_{\mathbb{K}} / \mathbb{K}^{*}, C_{\mathbb{K}}\right)$ according to projectors associated to characters of $C_{\mathbb{K}, 1}$. 
Proposition 4.12. Characters $\chi \in \widehat{C_{\mathbb{K}}, 1}$ determine a canonical direct sum decomposition

$$
\begin{gathered}
H^{1}\left(\mathbb{A}_{\mathbb{K}} / \mathbb{K}^{*}, C_{\mathbb{K}}\right)=\bigoplus_{\chi \in \widehat{C_{\mathbb{K}, 1}}} H_{\chi}^{1}\left(\mathbb{A}_{\mathbb{K}} / \mathbb{K}^{*}, C_{\mathbb{K}}\right) \\
H_{\chi}^{1}\left(\mathbb{A}_{\mathbb{K}} / \mathbb{K}^{*}, C_{\mathbb{K}}\right)=\left\{\xi \mid \underline{\vartheta}_{m}(\gamma) \xi=\chi(\gamma) \xi, \forall \gamma \in C_{\mathbb{K}, 1}\right\} .
\end{gathered}
$$

where $\underline{\vartheta}_{m}(\gamma)$ denotes the induced action (4.34) on $H^{1}\left(\mathbb{A}_{\mathbb{K}} / \mathbb{K}^{*}, C_{\mathbb{K}}\right)$.

Proof. The action of $\mathbb{A}_{\mathbb{K}}^{*}$ on $\mathcal{H}^{1}\left(\mathbb{A}_{\mathbb{K}} / \mathbb{K}^{*}, C_{\mathbb{K}}\right)$ induces a corresponding action of $C_{\mathbb{K}}$ on $H^{1}\left(\mathbb{A}_{\mathbb{K}} / \mathbb{K}^{*}, C_{\mathbb{K}}\right)$.

We can then reformulate the result of [11] based on the trace formula of [10] in the formulation of [32] in terms of the cohomology $H^{1}\left(\mathbb{A}_{\mathbb{K}} / \mathbb{K}^{*}, C_{\mathbb{K}}\right)$ in the following way.

Proposition 4.13. The induced representation of $C_{\mathbb{K}}$ on $H_{\chi}^{1}\left(\mathbb{A}_{\mathbb{K}} / \mathbb{K}^{*}, C_{\mathbb{K}}\right)$ gives the spectral realization of the zeros of the L-function with Grössencharakter $\chi$.

This result is a variant of Corollary 2 of [10], the proof is similar and essentially reduces to the result of [32]. There is a crucial difference with [10] in that all zeros (including those not located on the critical line) now appear due to the choice of the function spaces. To see what happens it is simpler to deal with the dual spaces i.e. to compute the cyclic cohomology $H C^{0}$. Its elements are cyclic morphisms $T$ from $\mathcal{H}^{1}\left(\mathbb{A}_{\mathbb{K}} / \mathbb{K}^{*}, C_{\mathbb{K}}\right)$ to $\mathbb{C}^{\natural}$ and they are determined by the map $T^{0}$ in degree 0 . The cyclic morphism property then shows that $T^{0}$ defines a trace on $\mathbf{S}^{\natural}\left(C_{\mathbb{K}}, \mathcal{L}^{1}\left(\mathcal{H}_{x}\right)\right)$ which vanishes on the range of $\rho^{\natural}$. The freeness of the action of $\mathbb{K}^{*}$ on $\mathbb{A}_{\mathbb{K}}^{*}$ then ensures that these traces are given by continuous linear forms on $\mathbf{S}\left(C_{\mathbb{K}}\right)$ which vanish on the following subspace of $\mathbf{S}\left(C_{\mathbb{K}}\right)$ which is the range of the restriction map, defined as follows.

Definition 4.14. Let $\mathcal{V} \subset \mathbf{S}\left(C_{\mathbb{K}}\right)$ denote the range of the map $\operatorname{Tr} \circ \rho$, that is,

$$
\mathcal{V}=\left\{h \in \mathbf{S}\left(C_{\mathbb{K}}\right) \mid h(x)=\sum_{k \in \mathbb{K}^{*}} \xi(k x) \text {, with } \xi \in \mathcal{S}\left(\mathbb{A}_{\mathbb{K}}\right)_{0}\right\},
$$

where $\mathcal{S}\left(\mathbb{A}_{\mathbb{K}}\right)_{0}=\operatorname{Ker} \varepsilon_{0} \cap \operatorname{Ker} \varepsilon_{1} \subset \mathcal{S}\left(\mathbb{A}_{\mathbb{K}}\right)$.

We have seen above in the case $\mathbb{K}=\mathbb{Q}(c f .[10])$ that the range of $\operatorname{Tr} \circ \rho$ is indeed contained in $\mathbf{S}\left(C_{\mathbb{K}}\right)$.

Moreover, we have the following results about the action $\underline{\vartheta}_{m}(\gamma)$, for $\gamma \in C_{\mathbb{K}}$, on $H^{1}\left(\mathbb{A}_{\mathbb{K}} / \mathbb{K}^{*}, C_{\mathbb{K}}\right)$. Suppose given $f \in \mathbf{S}\left(C_{\mathbb{K}}\right)$. We define a corresponding operator

$$
\underline{\vartheta}_{m}(f)=\int_{C_{\mathbb{K}}} f(\gamma) \underline{\vartheta}_{m}(\gamma) d^{*} \gamma,
$$

acting on the complex vector space $H^{1}\left(\mathbb{A}_{\mathbb{K}} / \mathbb{K}^{*}, C_{\mathbb{K}}\right)$. Here $d^{*} \gamma$ is the multiplicative Haar measure on $C_{\mathbb{K}}$. We have the following description of the action of $\underline{\vartheta}_{m}(f)$. 
Lemma 4.15. For $f \in \mathbf{S}\left(C_{\mathbb{K}}\right)$, the action of the operator $\underline{\vartheta}_{m}(f)$ of (4.38) on $H^{1}\left(\mathbb{A}_{\mathbb{K}} / \mathbb{K}^{*}, C_{\mathbb{K}}\right)$ is the action induced on the quotient of $\mathbf{S}\left(C_{\mathbb{K}}\right)$ by $\mathcal{V} \subset \mathbf{S}\left(C_{\mathbb{K}}\right)$ of the action of $\vartheta_{m}(f)$ on $\mathbf{S}\left(C_{\mathbb{K}}\right)$ by convolution product

$$
\vartheta_{m}(f) \xi(u)=\int_{C_{\mathbb{K}}} \xi\left(g^{-1} u\right) f(g) d^{*} g=(f \star \xi)(u) .
$$

Proof. One first shows that one can lift $f$ to a function $\tilde{f}$ on $\mathbb{A}_{\mathbb{K}}^{*}$ such that

$$
\sum_{k \in \mathbb{K}^{*}} \tilde{f}(k x)=f(x)
$$

and that convolution by $\tilde{f}$ i.e.

$$
\int \tilde{f}(\gamma) \vartheta_{a}(\gamma) d^{*} \gamma
$$

leaves $\mathcal{S}\left(\mathcal{G}_{\mathbb{K}}\right)$ globally invariant. This means showing that that $\mathcal{S}\left(\mathbb{A}_{\mathbb{K}}\right)_{0}$ is stable under convolution by the lift of $\mathbf{S}\left(C_{\mathbb{K}}\right)$. Then (4.39) follows directly from the definition of the actions (4.33), (4.30), (4.34) and the operator (4.38).

For $f \in \mathbf{S}\left(C_{\mathbb{K}}\right)$ and $\tilde{\chi}$ the extension of a character $\chi \in \widehat{C_{\mathbb{K}, 1}}$ to $C_{\mathbb{K}}$ and $\hat{f}(\tilde{\chi}, \rho)$ the Fourier transform (2.30), the operators $\underline{\vartheta}_{m}(f)$ of (4.38) satisfy the spectral side of the trace formula. Namely, we have the following result.

Theorem 4.16. For any $f \in \mathbf{S}\left(C_{\mathbb{K}}\right)$, the operator $\underline{\vartheta}_{m}(f)$ defined in (4.38) acting on $H^{1}\left(\mathbb{A}_{\mathbb{K}} / \mathbb{K}^{*}, C_{\mathbb{K}}\right)$ is of trace class. The trace is given by

$$
\operatorname{Tr}\left(\underline{\vartheta}_{m}(f) \mid H^{1}\left(\mathbb{A}_{\mathbb{K}} / \mathbb{K}^{*}, C_{\mathbb{K}}\right)\right)=\sum_{\rho \in \mathbb{C} / N^{\perp} \mid L(\tilde{\chi}, \rho)=0} \hat{f}(\tilde{\chi}, \rho),
$$

with $\hat{f}(\tilde{\chi}, \rho)$ the Fourier transform (2.30).

Proof. Due to the different normalization of the summation map, the representation $\underline{\vartheta}_{m}(\gamma)$ considered here differs from the action $W(\gamma)$ considered in [10] by

$$
\underline{\vartheta}_{m}(\gamma)=|\gamma|^{1 / 2} W(\gamma) \text {. }
$$

This means that we have

$$
\underline{\vartheta}_{m}(f)=\int_{C_{\mathbb{K}}} f(\gamma) \underline{\vartheta}_{m}(\gamma) d^{*} \gamma=\int_{C_{\mathbb{K}}} h(\gamma) W(\gamma) d^{*} \gamma,
$$

where

$$
h(\gamma)=|\gamma|^{1 / 2} f(\gamma)
$$

We then have, for $W(h)=\int_{C_{\mathbb{K}}} h(\gamma) W(\gamma) d^{*} \gamma$,

$$
\operatorname{Tr} W(h)=\sum_{\rho \in \mathbb{C} / N^{\perp} \mid L\left(\tilde{\chi}, \frac{1}{2}+\rho\right)=0} \hat{h}(\tilde{\chi}, \rho) .
$$


Note that unlike in [10] all zeros contribute, including those that might fail to be on the critical line, and they do with their natural multiplicity. This follows from the choice of function space as in [32]. The Fourier transform $\hat{h}(\tilde{\chi}, \rho)$ satisfies

$$
\hat{h}(\tilde{\chi}, \rho)=\int_{C_{\mathbb{K}}} h(u) \tilde{\chi}(u)|u|^{\rho} d^{*} u=\int_{C_{\mathbb{K}}} f(u) \tilde{\chi}(u)|u|^{\rho+1 / 2} d^{*} u=\hat{f}(\tilde{\chi}, \rho+1 / 2),
$$

where $h$ and $f$ are related as in (4.43). Thus, the shift by $1 / 2$ in (4.44) is absorbed in (4.45) and this gives the required formula (4.40).

4.5. Trace pairing and vanishing. The commutativity of the convolution product implies the following vanishing result.

Lemma 4.17. Suppose given an element $f \in \mathcal{V} \subset \mathbf{S}\left(C_{\mathbb{K}}\right)$, where $\mathcal{V}$ is the range of the reduction map as in Definition 4.14. Then one has

$$
\left.\underline{\vartheta}_{m}(f)\right|_{H^{1}\left(\mathbb{A}_{\mathbb{K}} / \mathbb{K}^{*}, C_{\mathbb{K}}\right)}=0 \text {. }
$$

Proof. The result follows by showing that, for $f \in \mathcal{V}$, the operator $\underline{\vartheta}_{m}(f)$ maps any element $\xi \in \mathbf{S}\left(C_{\mathbb{K}}\right)$ to an element in $\mathcal{V}$, hence the induced map on the quotient of $\mathbf{S}\left(C_{\mathbb{K}}\right)$ by $\mathcal{V}$ is trivial. Since $\mathcal{V}$ is a submodule of $\mathbf{S}\left(C_{\mathbb{K}}\right)$ for the action of $\mathbf{S}\left(C_{\mathbb{K}}\right)$ by convolution we obtain

$$
\underline{\vartheta}_{m}(f) \xi=f \star \xi=\xi \star f \in \mathcal{V},
$$

where $\star$ is the convolution product of (4.39).

This makes it possible to define a trace pairing as follows.

Remark 4.18. The pairing

$$
f_{1} \otimes f_{2} \mapsto\left\langle f_{1}, f_{2}\right\rangle_{H^{1}}:=\operatorname{Tr}\left(\underline{\vartheta}_{m}\left(f_{1} \star f_{2}\right) \mid H^{1}\left(\mathbb{A}_{\mathbb{K}} / \mathbb{K}^{*}, C_{\mathbb{K}}\right)\right)
$$

descends to a well defined pairing on $H^{1}\left(\mathbb{A}_{\mathbb{K}} / \mathbb{K}^{*}, C_{\mathbb{K}}\right) \otimes H^{1}\left(\mathbb{A}_{\mathbb{K}} / \mathbb{K}^{*}, C_{\mathbb{K}}\right)$.

\section{Primitive Cohomology}

The aim of this section is to interpret the motivic construction described in the previous paragraph as the noncommutative version of a classical construction in algebraic geometry. In motive theory, realizations of (mixed) motives appear frequently in the form of kernels/cokernels of relevant homomorphisms. The primitive cohomology is the example we shall review hereafter.

If $Y$ is a compact Kähler variety, a Kähler cocycle class $[\omega] \in H^{2}(Y, \mathbb{R})$ determines the Lefschetz operator $\left(i \in \mathbb{Z}_{\geq 0}\right)$ :

$$
L: H^{i}(Y, \mathbb{R}) \rightarrow H^{i+2}(Y, \mathbb{R}), \quad L(a):=[\omega] \cup a .
$$

Let $n=\operatorname{dim} Y$. Then, the primitive cohomology is defined as the kernel of iterated powers of the Lefschetz operator

$$
H^{i}(Y, \mathbb{R})_{\text {prim }}:=\operatorname{Ker}\left(L^{n-i+1}: H^{i}(Y, \mathbb{R}) \rightarrow H^{2 n-i+2}(Y, \mathbb{R})\right) .
$$


In particular, for $i=n$ we have

$$
H^{n}(Y, \mathbb{R})_{\text {prim }}:=\operatorname{Ker}\left(L: H^{n}(Y, \mathbb{R}) \rightarrow H^{n+2}(Y, \mathbb{R})\right) .
$$

Let assume, from now on, that $j: Y \hookrightarrow X$ is a smooth hyperplane section of a smooth, projective complex algebraic variety $X$. Then, it is a classical result of geometric topology that $L=j^{*} \circ j_{*}$, where

$$
j_{*}: H^{i}(Y, \mathbb{R}) \rightarrow H^{i+2}(X, \mathbb{R})
$$

is the Gysin homomorphism: the Poincaré dual of the restriction homomorphism

$$
j^{*}: H^{2 n-i}(X, \mathbb{R}) \rightarrow H^{2 n-i}(Y, \mathbb{R}) .
$$

In fact, because the class of $L$ comes from an integral class, the equality $L=j^{*} \circ j_{*}$ holds already in integral cohomology. For $i=n$, the above description of the Lefschetz operator together with the Lefschetz theorem of hyperplane sections imply that

$$
H^{n}(Y, \mathbb{R})_{\text {prim }} \cong \operatorname{Ker}\left(j_{*}: H^{n}(Y, \mathbb{R}) \rightarrow H^{n+2}(X, \mathbb{R})\right)=: H^{n}(Y, \mathbb{R})_{\text {van }}
$$

where by $H^{i}(Y, \mathbb{R})_{\text {van }}$ we denote the vanishing cohomology

$$
H^{i}(Y, \mathbb{R})_{\text {van }}:=\operatorname{Ker}\left(j_{*}: H^{i}(Y, \mathbb{R}) \rightarrow H^{i+2}(X, \mathbb{R})\right) .
$$

Now, we introduce the theory of mixed Hodge structures in this set-up.

Let $U:=X \backslash Y$ be the open space which is the complement of $Y$ in $X$ and let denote by $k: U \hookrightarrow X$ the corresponding open immersion. Then, one knows that $R^{i} j_{*} \mathbb{Z}=0$ unless $i=0,1$ so that the Leray spectral sequence for $j$ :

$$
E_{2}^{p, q}=H^{q}\left(X, \mathrm{R}^{p} k_{*} \mathbb{Z}\right) \Rightarrow H^{p+q}(U, \mathbb{Z})
$$

coincides with the long exact sequence (of mixed Hodge structures)

$$
\ldots \stackrel{\partial}{\rightarrow} H^{i-2}(Y, \mathbb{Z})(-1) \stackrel{j_{*}}{\rightarrow} H^{i}(X, \mathbb{Z}) \rightarrow H^{i}(U, \mathbb{Z}) \stackrel{\partial}{\rightarrow} \ldots
$$

The boundary homomorphism $\partial$ in this sequence is known to coincide ([21], § 9.2) with the residue homomorphism

$$
\text { Res }: H^{i+1}(U, \mathbb{Z}) \rightarrow H^{i}(Y, \mathbb{Z})(-1)
$$

whose description, with complex coefficients, is derived from a corresponding morphism of filtered complexes (Poincaré residue map). This morphism fits in the following exact sequence of filtered complexes of Hodge modules

$$
\begin{aligned}
0 \rightarrow \Omega_{X} \rightarrow & \Omega_{X}(\log Y) \stackrel{\text { res }}{\rightarrow} j_{*} \Omega_{Y}[-1] \rightarrow 0 \\
& \operatorname{res}\left(\alpha \wedge \frac{d t}{t}\right)=\alpha_{\mid Y} .
\end{aligned}
$$

One knows that Res is a homomorphism of Hodge structures, hence the Hodge filtration on $H^{n+1}(U, \mathbb{C}) \cong \mathbb{H}^{n+1}\left(X, \Omega_{X}(\log Y)\right)$ determines a corresponding filtration on the (twisted) vanishing cohomology

$H^{n}(Y, \mathbb{C})(n)_{\text {van }}=\operatorname{Ker}\left(j_{*}: H^{n}(Y, \mathbb{C})(n) \rightarrow H^{n+2}(X, \mathbb{C})(n+1)\right) \cong H^{n+1}(U)(n+1)$. 
In degree $i=n$, one also knows that the excision exact sequence (of Hodge structures) becomes the short exact sequence

$$
0 \rightarrow H^{n}(X, \mathbb{C}) \stackrel{j^{*}}{\rightarrow} H^{n}(Y, \mathbb{C}) \rightarrow H_{c}^{n+1}(U, \mathbb{C}) \rightarrow 0 .
$$

Therefore, it follows by the Poincaré duality isomorphism

$$
H_{c}^{n+1}(U, \mathbb{C})^{*} \cong H^{n+1}(U, \mathbb{C})(n+1)
$$

that

$$
\left(\operatorname{Coker}\left(j^{*}: H^{n}(X, \mathbb{C}) \rightarrow H^{n}(Y, \mathbb{C})\right)\right)^{*} \cong H_{c}^{n+1}(U, \mathbb{C})^{*} \cong H^{n}(Y, \mathbb{C})(n)_{\text {van }}
$$

When $j: Y \hookrightarrow X$ is a singular hypersurface or a divisor in $X$ with (local) normal crossings (i.e.: $Y=\bigcup_{i} Y_{i}, \operatorname{dim} Y_{i}=n=\operatorname{dim} X-1, Y$ locally described by an equation $x_{i_{1}} \cdots x_{i_{r}}=0,\left\{i_{1}, \ldots i_{r}\right\} \subseteq\{1, \ldots n+1\},\left\{x_{1}, \ldots x_{n+1}\right\}=$ system of local coordinates in $X$ ), the notion of the Gysin homomorphism is lost. One then replaces the vanishing cohomology by the primitive cohomology, whose definition extends to this general set-up and is given, in analogy to (5.1), as

$$
H^{n}(Y, \mathbb{C})_{\text {prim }}:=\operatorname{Coker}\left(j^{*}: H^{n}(X, \mathbb{C}) \rightarrow H^{n}(Y, \mathbb{C})\right) \subseteq H_{c}^{n+1}(U, \mathbb{C}) .
$$

One also knows that the primitive cohomology is motivic ( $c f$. 22] and [3] for interesting examples). Following the classical construction that we have just reviewed, we like to argue now that the definition of the cyclic module $\mathcal{H}^{1}\left(\mathbb{A}_{\mathbb{K}} / \mathbb{K}^{*}, C_{\mathbb{K}}\right.$ ) (as in Definition 4.10), which is based on a noncommutative version of a restriction map "from adeles to ideles" defined in the category of $\Lambda$-modules, should be interpreted as the noncommutative analogue of a primitive motive (a cyclic primitive module). The cohomological realization of such motive (i.e. its cyclic homology) is given by the group $H^{1}\left(\mathbb{A}_{\mathbb{K}} / \mathbb{K}^{*}, C_{\mathbb{K}}\right)=\operatorname{Tor}\left(\mathbb{C}^{\natural}, \mathcal{H}^{1}\left(\mathbb{A}_{\mathbb{K}} / \mathbb{K}^{*}, C_{\mathbb{K}}\right)\right)(c f$. (4.28) $)$ which therefore can be interpreted as a noncommutative version of a primitive cohomology.

\section{A cohomological Lefschetz trace formula}

6.1. Weil's explicit formula as a trace formula. As in $\$ 2.2$ above, let $\alpha$ be a non-trivial character of $\mathbb{A}_{\mathbb{K}}$ which is trivial on $\mathbb{K} \subset \mathbb{A}_{\mathbb{K}}$. It is well known ([43] VII-2) that for such a character $\alpha$ there exists a differental idele $a=\left(a_{v}\right) \in \mathbb{A}_{\mathbb{K}}^{*}$ such that

$$
\alpha_{v}(x)=e_{\mathbb{K}_{v}}\left(a_{v} x\right), \quad \forall x \in \mathbb{K}_{v},
$$

where, for a local field $K$, the additive character $e_{K}$ is chosen in the following way.

- If $K=\mathbb{R}$ then $e_{\mathbb{R}}(x)=e^{-2 \pi i x}$, for all $x \in \mathbb{R}$.

- If $K=\mathbb{C}$ then $e_{\mathbb{C}}(z)=e^{-2 \pi i(z+\bar{z})}$, for all $z \in \mathbb{C}$.

- If $K$ is a non-archimedean local field with maximal compact subring $\mathcal{O}$, then the character $e_{K}$ satisfies $\operatorname{Ker} e_{K}=\mathcal{O}$. 
The notion of differental idele can be thought of as an extension of the canonical class of the algebraic curve $C$, from the setting of function fields $\mathbb{F}_{q}(C)$ to arbitrary global fields $\mathbb{K}$. For instance, one has

$$
|a|=q^{2-2 g} \quad \text { or } \quad|a|=D^{-1},
$$

respectively, for the case of a function field $\mathbb{F}_{q}(C)$ and of a number field. In the number field case $D$ denotes the discriminant.

In [11] we gave a cohomological formulation of the Lefschetz trace formula of [10], using the version of the Riemann-Weil explicit formula as a trace formula given in 32 in the context of nuclear spaces, rather than the semi-local Hilbert space version of [10].

Theorem 6.1. For $f \in \mathbf{S}\left(C_{\mathbb{K}}\right)$ let $\underline{\vartheta}_{m}(f)$ be the operator (4.38) acting on the space $H^{1}=H^{1}\left(\mathbb{A}_{\mathbb{K}} / \mathbb{K}^{*}, C_{\mathbb{K}}\right)$. Then the trace is given by

$$
\operatorname{Tr}\left(\underline{\vartheta}_{m}(f) \mid H^{1}\right)=\hat{f}(0)+\hat{f}(1)-(\log |a|) f(1)-\sum_{v \in \Sigma_{\mathbb{K}}} \int_{\left(\mathbb{K}_{v}^{*}, e_{\mathbb{K}_{v}}\right)}^{\prime} \frac{f\left(u^{-1}\right)}{|1-u|} d^{*} u .
$$

The formula (6.3) is obtained in [11] first by showing that the Lefschetz trace formula of [10] in the version of [32] can be formulated equivalently in the form

$$
\operatorname{Tr}\left(\underline{\vartheta}_{m}(f) \mid H^{1}\right)=\hat{f}(0)+\hat{f}(1)-\sum_{v \in \mathbb{K}_{v}} \int_{\mathbb{K}_{v}^{*}}^{\prime} \frac{f\left(u^{-1}\right)}{|1-u|} d^{*} u,
$$

where one uses the global character $\alpha$ to fix the local normalizations of the principal values in the last term of the formula. We then compute this principal value using the differental idele in the form

$$
\int_{\left(\mathbb{K}_{v}^{*}, \alpha_{v}\right)}^{\prime} \frac{f\left(u^{-1}\right)}{|1-u|} d^{*} u=\left(\log \left|a_{v}\right|\right) f(1)+\int_{\left(\mathbb{K}_{v}^{*}, e_{\mathbb{K}_{v}}\right)}^{\prime} \frac{f\left(u^{-1}\right)}{|1-u|} d^{*} u
$$

6.2. Weil Positivity and the Riemann Hypothesis. We introduce an involution for elements $f \in \mathbf{S}\left(C_{\mathbb{K}}\right)$ by setting

$$
f^{*}(g)=\overline{f\left(g^{-1}\right)}
$$

We also consider a one parameter group $z \mapsto \Delta^{z}$ of automorphisms of the convolution algebra $\mathbf{S}\left(C_{\mathbb{K}}\right)$, with the convolution product (4.39) by setting

$$
\Delta^{z}(f)(g)=|g|^{z} f(g),
$$

for $f \in \mathbf{S}\left(C_{\mathbb{K}}\right)$ and $z \in \mathbb{C}$. Since (6.7) is given by multiplication by a character, it satisfies

$$
\Delta^{z}(f \star h)=\Delta^{z}(f) \star \Delta^{z}(f), \quad \forall f, h \in \mathbf{S}\left(C_{\mathbb{K}}\right) .
$$

We consider also the involution

$$
f \mapsto f^{\sharp}=\Delta^{-1} f^{*}, \quad \text { with } \quad f^{\sharp}(g)=|g|^{-1} \overline{f\left(g^{-1}\right)} .
$$

The reformulation, originally due to A. Weil, of the Riemann Hypothesis in our setting is given by the following statement. 
Proposition 6.2. The following two conditions are equivalent:

- All L-functions with Grössencharakter on $\mathbb{K}$ satisfy the Riemann Hypothesis.

- The trace pairing (4.47) satisfies the positivity condition

$$
\left\langle\Delta^{-1 / 2} f, \Delta^{-1 / 2} f^{*}\right\rangle \geq 0, \quad \forall f \in \mathbf{S}\left(C_{\mathbb{K}}\right) .
$$

Proof. Let $W(\gamma)=|\gamma|^{-1 / 2} \underline{\vartheta}_{m}(\gamma)$. Then, by [41] the RH for $L$-functions with Grössencharakter on $\mathbb{K}$ is equivalent to the positivity

$$
\operatorname{Tr}\left(W\left(f \star f^{*}\right)\right) \geq 0, \quad \forall f \in \mathbf{S}\left(C_{\mathbb{K}}\right) .
$$

Thus, in terms of the representation $\underline{\vartheta}_{m}$ we are considering here, we have

$$
W(f)=\underline{\vartheta}_{m}\left(\Delta^{-1 / 2} f\right) .
$$

Using the multiplicative property (6.8) of $\Delta^{z}$ we rewrite (6.11) in the equivalent form (6.10).

In terms of the involution (6.9) we can reformulate Proposition 6.2 in the following equivalent way.

Corollary 6.3. The following conditions are equivalent

- All L-functions with Grössencharakter on $\mathbb{K}$ satisfy the Riemann Hypothesis.

- The trace pairing (4.47) satisfies $\left\langle f, f^{\sharp}\right\rangle \geq 0$, for all $f \in \mathbf{S}\left(C_{\mathbb{K}}\right)$.

Proof. In (6.10) we write $\Delta^{-1 / 2} f=h$. This gives

$$
\Delta^{-1 / 2} f^{*}=\Delta^{-1 / 2}\left(\Delta^{1 / 2} h\right)^{*}=\Delta^{-1} h^{*}=h^{\sharp}
$$

and the result follows, since $\Delta^{-1 / 2}$ is an automorphism of $\mathbf{S}\left(C_{\mathbb{K}}\right)$.

The vanishing result of Lemma 4.17, for elements in the range $\mathcal{V} \subset \mathbf{S}\left(C_{\mathbb{K}}\right)$ of the reduction map $\operatorname{Tr} \circ \rho$ from adeles, gives then the following result.

Proposition 6.4. The elements $f \star f^{\sharp}$ considered in Corollary 6.3 above have the following properties.

(1) The trace pairing $\left\langle f, f^{\sharp}\right\rangle$ vanishes for all $f \in \mathcal{V}$, i.e. when $f$ is the restriction $\operatorname{Tr} \circ \rho$ of an element of $\mathcal{S}\left(\mathcal{G}_{\mathbb{K}}\right)$.

(2) By adding elements of $\mathcal{V}$ one can make the values

$$
f \star f^{\sharp}(1)=\int_{C_{\mathbb{K}}}|f(g)|^{2}|g| d^{*} g<\epsilon
$$

for arbitrarily small $\epsilon>0$.

Proof. (1) The vanishing result of Lemma 4.17 shows that $\left.\underline{\vartheta}_{m}(f)\right|_{H^{1}\left(\mathbb{A}_{\mathbb{K}} / \mathbb{K}^{*}, C_{\mathbb{K}}\right)}=0$ for all $f \in \mathcal{V}$. Thus, the trace pairing satisfies $\langle f, h\rangle=0$, for $f \in \mathcal{V}$ and for all $h \in \mathbf{S}\left(C_{\mathbb{K}}\right)$. In particular this applies to the case $h=f^{\sharp}$.

(2) This follows from the surjectivity of the map $\mathfrak{E}$ for the weight $\delta=0$ (cf. Appendix 1 of [10]). 
Proposition 6.4 shows that the trace pairing admits a large radical given by all functions that extend to adeles. Thus, one can divide out this radical and work with the cohomology $H^{1}\left(\mathbb{A}_{\mathbb{K}} / \mathbb{K}^{*}, C_{\mathbb{K}}\right)$ described above.

\section{Correspondences}

To start building the dictionary between the Weil proof and the noncommutative geometry of the adeles class space, we begin by reformulating the trace formula discussed above in more intersection theoretic language, so as to be able to compare it with the setup of $\$ 2.1$ above. We also discuss in this section the analog of moding out by trivial correspondence.

7.1. The scaling correspondence as Frobenius. To the scaling action

$$
\vartheta_{a}(\gamma)(\xi)(x)=\xi\left(\gamma^{-1} x\right)
$$

one associates the graph $Z_{g}$ given by the pairs $\left(x, g^{-1} x\right)$. These should be considered as points in the product $\mathbb{A}_{\mathbb{K}} / \mathbb{K}^{*} \times \mathbb{A}_{\mathbb{K}} / \mathbb{K}^{*}$ of two copies of the adeles class space. Thus, the analog in our context of the correspondences $Z=\sum_{n} a_{n} \mathrm{Fr}^{n}$ on $C \times C$ is given by elements of the form

$$
Z(f)=\int_{C_{\mathbb{K}}} f(g) Z_{g} d^{*} g,
$$

for some $f \in \mathbf{S}\left(C_{\mathbb{K}}\right)$.

With this interpretation of correspondences, we can then make sense of the terms in the trace formula in the following way.

Definition 7.1. For a correspondence of the form (7.1) we define degree and codegree by the following prescription

$$
\begin{gathered}
d(Z(f)):=\hat{f}(1)=\int_{C_{\mathbb{K}}} f(u)|u| d^{*} u, \\
d^{\prime}(Z(f)):=d\left(Z\left(f^{\sharp}\right)\right)=\int_{C_{\mathbb{K}}} f(u) d^{*} u=\hat{f}(0) .
\end{gathered}
$$

Here the Fourier transform $\hat{f}$ is as in (2.30), with the trivial character $\chi=1$. Notice that, with this definition of degree and codegree we find

$$
d\left(Z_{g}\right)=|g|, \quad \text { and } \quad d^{\prime}\left(Z_{g}\right)=1 .
$$

Thus, the term $\hat{f}(1)+\hat{f}(0)$ in the trace formula of Theorem 6.1 match the term $d(Z)+d^{\prime}(Z)$ in Weil's formula for the trace of a correspondence as in (2.25). The term

$$
-\int_{\left(\mathbb{K}_{v}^{*}, \alpha_{v}\right)}^{\prime} \frac{f\left(u^{-1}\right)}{|1-u|} d^{*} u
$$

of (6.4) in turn can be seen as the remaining term $-Z \bullet \Delta$ in (2.25). In fact, the formula (7.5) describes, using distributions, the local contributions to the trace of the intersections between the graph $Z(f)$ and the diagonal $\Delta$. This was proved in [10], Section VI and Appendix III. It generalizes the analogous formula for 
flows on manifolds of [23, which in turn can be seen as a generalization of the usual Atiyah-Bott Lefschetz formula for a diffeomorphism of a smooth compact manifold [2].

When we separate out the contribution $\log |a| h(1)$, as in passing from (6.4) to (6.3), and we rewrite the trace formula as in Theorem 6.1. This corresponds to separating the intersection $Z \bullet \Delta$ into a term that is proportional to the self intersection $\Delta \bullet \Delta$ and a remaning terms where the intersection is transverse.

To see this, we notice that the term $\log |a|$, for $a=\left(a_{v}\right)$ a differental idele, is of the form (6.2). Indeed one sees that, in the function field case the term

$$
-\log |a|=-\log q^{2-2 g}=(2 g-2) \log q=-\Delta \bullet \Delta \log q
$$

is proportional to the self intersection of the diagonal, which brings us to consider the value $\log |a|=-\log D$ with the discriminant of a number field as the analog in characteristic zero of the self intersection of the diagonal.

In these intersection theoretic terms we can reformulate the positivity condition ( $c f$. 4]) equivalent to the Riemann Hypothesis in the following way.

Proposition 7.2. The following two conditions are equivalent

- All L-functions with Grössencharakter on $\mathbb{K}$ satisfy the Riemann Hypothesis.

- The estimate

$$
Z(f) \bullet_{\text {trans }} Z(f) \leq 2 d(Z(f)) d^{\prime}(Z(f))-\Delta \bullet \Delta f \star f^{\sharp}(1)
$$

holds for all $f \in \mathbf{S}\left(C_{\mathbb{K}}\right)$.

Proof. As in the Weil proof one separates the terms $Z \star Z^{\prime}=d^{\prime}(Z) \Delta+Y$, where $Y$ has transverse intersection with the diagonal, here we can write an identity

$$
\operatorname{Tr}\left(\underline{\vartheta}_{m}\left(f \star f^{\sharp}\right) \mid H^{1}\right)=: Z(f) \bullet Z(f)=\Delta \bullet \Delta f \star f^{\sharp}(1)+Z(f) \bullet \text { trans } Z(f)
$$

where the remaning term $Z(f) \bullet$ trans $Z(f)$ which represents the transverse intersection is given by the local contributions given by the principal values over $\left(\mathbb{K}_{v}^{*}, e_{\mathbb{K}_{v}}\right)$ in the formula ( $(\underline{6.3})$ for $\operatorname{Tr}\left(\underline{\vartheta}_{m}\left(f \star f^{\sharp}\right) \mid H^{1}\right)$.

The formula (6.3) for $\operatorname{Tr}\left(\underline{\vartheta}_{m}\left(f \star f^{\sharp}\right) \mid H^{1}\right)$ gives a term of the form $-\log |a| f \star f^{\sharp}(1)$, with

$$
f \star f^{\sharp}(1)=\int_{C_{\mathbb{K}}}|f(g)|^{2}|g| d^{*} g .
$$

We rewrite this term as $-\Delta \bullet \Delta f \star f^{\sharp}(1)$ according to our interpretation of $\log |a|$ as self-intersection of the diagonal. This matches the term $(2 g-2) d^{\prime}(Z)$ in the estimate for $\operatorname{Tr}\left(Z \star Z^{\prime}\right)$ in the Weil proof.

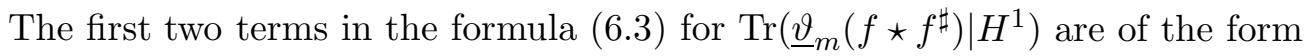

$$
\widehat{f \star f^{\sharp}}(0)+\widehat{f \star f^{\sharp}}(1)=2 \hat{f}(0) \hat{f}(1)=2 d^{\prime}(Z(f)) d(Z(f)) .
$$

This matches the term $2 g d^{\prime}(Z)=2 d(Z) d^{\prime}(Z)$ in the expression for $\operatorname{Tr}\left(Z \star Z^{\prime}\right)$ in the Weil proof.

With this notation understood, we see that the positivity $\operatorname{Tr}\left(\underline{\vartheta}_{m}\left(f \star f^{\sharp}\right) \mid H^{1}\right) \geq 0$ corresponds indeed to the estimate (7.6). 
7.2. Fubini's theorem and the trivial correspondences. As we have seen in recalling the main steps in the Weil proof, a first step in dealing with correspondences is to use the freedom to add multiples of the trivial correspondences in order to adjust the degree. We describe an analog, in our noncommutative geometry setting, of the trivial correspondences and of this operation of modifying the degree.

In view of the result of Proposition 6.4 above, it is natural to regard the elements $f \in \mathcal{V} \subset \mathbf{S}\left(C_{\mathbb{K}}\right)$ as those that give rise to the trivial correspondences $Z(f)$. Here, as above, $\mathcal{V}$ is the range of the reduction map from adeles.

The fact that it is possible to arbitrarily modify the degree $d(Z(f))=\hat{f}(1)$ of a correspondence by adding to $f$ an element in $\mathcal{V}$ depends on the subtle fact that we deal with a case where the Fubini theorem does not apply.

In fact, consider an element $\xi \in \mathcal{S}\left(\mathbb{A}_{\mathbb{K}}\right)_{0}$. We know that it satisfies the vanishing condition

$$
\int_{\mathbb{A}_{\mathbb{K}}} \xi(x) d x=0 .
$$

Thus, at first sight it would appear that, for the function on $C_{\mathbb{K}}$ defined by $f(x)=\sum_{k \in \mathbb{K}^{*}} \xi(k x)$,

$$
\hat{f}(1)=\int_{C_{\mathbb{K}}} f(g)|g| d^{*} g
$$

should also vanish, since we have $f(x)=\sum_{k \in \mathbb{K}^{*}} \xi(k x)$ and for local fields (but not in the global case) the relation between the additive and multiplicative Haar measures is of the form $d g=|g| d^{*} g$. This, however, is in general not the case. To see more clearly what happens, let us just restrict to the case $\mathbb{K}=\mathbb{Q}$ and assume that the function $\xi(x)$ is of the form

$$
\xi=\mathbf{1}_{\hat{\mathbb{Z}}} \otimes \eta
$$

with $\mathbf{1}_{\hat{\mathbb{Z}}}$ the characteristic function of $\hat{\mathbb{Z}}$ and with $\eta \in \mathcal{S}(\mathbb{R})_{0}$. We then have $C_{\mathbb{Q}}=\hat{\mathbb{Z}}^{*} \times \mathbb{R}_{+}^{*}$ and the function $f$ is of the form

$$
f(u, \lambda)=\sum_{n \in \mathbb{Z}, n \neq 0} \eta(n \lambda), \quad \forall \lambda \in \mathbb{R}_{+}^{*}, u \in \hat{\mathbb{Z}}^{*} .
$$

We can thus write (7.9) in this case as

$$
\hat{f}(1)=\int_{\hat{\mathbb{Z}}^{*} \times \mathbb{R}_{+}^{*}} f(u, \lambda) d u d \lambda=\int_{\mathbb{R}} \sum_{n \in \mathbb{N}} \eta(n \lambda) d \lambda
$$

Moreover since $\eta \in \mathcal{S}(\mathbb{R})_{0}$ we have for all $n$,

$$
\int_{\mathbb{R}} \eta(n \lambda) d \lambda=0
$$


It is however not necessarily the case that we can apply Fubini's theorem and write

$$
\int_{\mathbb{R}} \sum_{n \in \mathbb{N}} \eta(n \lambda) d \lambda=\sum_{n} \int_{\mathbb{R}} \eta(n \lambda) d \lambda=0
$$

since as soon as $\eta \neq 0$ one has

$$
\sum_{n=1}^{\infty} \int_{\mathbb{R}}|\eta(n \lambda)| d \lambda=\left(\int_{\mathbb{R}}|\eta(\lambda)| d \lambda\right) \sum_{n=1}^{\infty} \frac{1}{n}=\infty
$$

so that Fubini's theorem does not apply and one cannot interchange the integral and the sum in (7.13). Thus, one can in general have $\hat{f}(1) \neq 0$, even though $\sum_{n} \int_{\mathbb{R}} \eta(n \lambda) d \lambda=0$. In fact, we have the following result.

Lemma 7.3. Given $f \in \mathbf{S}\left(C_{\mathbb{K}}\right)$, it is possible to change arbitrarily the value of the degree $d(Z(f))=\hat{f}(1)$ by adding elements of $\mathcal{V}$.

Proof. It suffices to exhibit an element $f \in \mathcal{V}$ such that $\hat{f}(1) \neq 0$, as then by linearity one can obtain the result. We only treat the case $\mathbb{K}=\mathbb{Q}$. We take $\eta \in \mathcal{S}(\mathbb{R})_{0}$ given by

$$
\eta(x)=\pi x^{2}\left(\pi x^{2}-\frac{3}{2}\right) e^{-\pi x^{2}}
$$

One finds that, up to normalization, the Fourier transform $\hat{f}$ is given by

$$
\hat{f}(i s)=\int_{\mathbb{R}_{+}^{*}} \sum_{n \in \mathbb{N}} \eta(n \lambda) \lambda^{i s} d^{*} \lambda=s(s+i) \zeta^{*}(i s)
$$

where $\zeta^{*}$ is the complete zeta function,

$$
\zeta^{*}(z)=\pi^{-z / 2} \Gamma\left(\frac{z}{2}\right) \zeta(z) .
$$

This function has a simple pole at $z=1$ thus one gets that $\hat{f}(1) \neq 0$.

An important question, in order to proceed and build a dictionary that parallels the main steps in the Weil proof, is to identify the correct notion of principal divisors. To this purpose, we show that we have at least a good analog for the points of the curve, in terms of states of some thermodynamical systems, that extend from the function field setting to the number field case.

\section{Thermodynamics AND GEOMETRY OF THE PRIMES}

Let $\mathbb{K}$ be a global field, with $\mathbb{A}_{\mathbb{K}}$ the ring of adeles and $C_{\mathbb{K}}$ the ideles classes, as above. We denote by $C_{\mathbb{K}, 1} \subset C_{\mathbb{K}}$ the kernel of the norm $|\cdot|: C_{\mathbb{K}} \rightarrow \mathbb{R}_{+}^{*}$.

The origin ( $c f .[10]$ ) of the terms in the geometric side of the trace formula (Theorem 6.1) comes from the Lefschetz formula by Atiyah-Bott 2 and its adaptation by Guillemin-Sternberg ( $c f$. [23]) to the distribution theoretic trace for flows on manifolds, which is a variation on the theme of [2]. For the action of $C_{\mathbb{K}}$ on the adele class space $X_{\mathbb{K}}$ the relevant periodic points are

$$
P=\left\{(x, u) \in X_{\mathbb{K}} \times C_{\mathbb{K}} \mid u x=x\right\}
$$


and one has (cf. [10])

Proposition 8.1. Let $(x, u) \in P$, with $u \neq 1$. There exists a place $v \in \Sigma_{\mathbb{K}}$ such that

$$
x \in X_{\mathbb{K}, v}=\left\{x \in X_{\mathbb{K}} \mid x_{v}=0\right\}
$$

The isotropy subgroup of any $x \in X_{\mathbb{K}, v}$ contains the cocompact subgroup

$$
\mathbb{K}_{v}^{*} \subset C_{\mathbb{K}}, \quad \mathbb{K}_{v}^{*}=\left\{\left(k_{w}\right) \mid k_{w}=1 \forall w \neq v\right\}
$$

The spaces $X_{\mathbb{K}, v}$ are noncommutative spaces, as such they are described by the following noncommutative algebras:

Definition 8.2. Let $\mathbb{A}_{\mathbb{K}, v} \subset \mathbb{A}_{\mathbb{K}}$ denote the closed $\mathbb{K}^{*}$-invariant subset of adeles

$$
\mathbb{A}_{\mathbb{K}, v}=\left\{a=\left(a_{w}\right)_{w \in \Sigma_{\mathbb{K}}} \mid a_{v}=0\right\} .
$$

Let $\mathcal{G}_{\mathbb{K}, v}$ denote the closed subgroupoid of $\mathcal{G}_{\mathbb{K}}$ given by

$$
\mathcal{G}_{\mathbb{K}, v}=\left\{(k, x) \in \mathcal{G}_{\mathbb{K}} \mid x_{v}=0\right\},
$$

and let $\mathcal{A}_{v}=\mathcal{S}\left(\mathcal{G}_{\mathbb{K}, v}\right)$ be the corresponding groupoid algebra.

Since the inclusion $\mathbb{A}_{\mathbb{K}, v} \subset \mathbb{A}_{\mathbb{K}}$ is $\mathbb{K}^{*}$-equivariant and proper, it extends to an algebra homomorphism

$$
\rho_{v}: \mathcal{S}\left(\mathcal{G}_{\mathbb{K}}\right) \rightarrow \mathcal{S}\left(\mathcal{G}_{\mathbb{K}, v}\right)
$$

which plays the role of the restriction map to the periodic orbit $X_{\mathbb{K}, v}$. We shall now determine the classical points of each of the $X_{\mathbb{K}, v}$. Taken together these will form the following locus inside the adeles class space, which we refer to as the "periodic classical points" of $X_{\mathbb{K}}=\mathbb{A}_{\mathbb{K}} / \mathbb{K}^{*}$.

Definition 8.3. Let $\mathbb{K}$ be a global field. For a place $v \in \Sigma_{\mathbb{K}}$ consider the adele

$$
a^{(v)}=\left(a_{w}^{(v)}\right), \quad \text { with } \quad a_{w}^{(v)}=\left\{\begin{array}{cc}
1 & w \neq v \\
0 & w=v .
\end{array}\right.
$$

The set of periodic classical points of the adeles class space $\mathbb{A}_{\mathbb{K}} / \mathbb{K}^{*}$ is defined as the union of orbits

$$
\Xi_{\mathbb{K}}:=\bigcup_{v \in \Sigma_{\mathbb{K}}} C_{\mathbb{K}} a^{(v)}
$$

8.1. The global Morita equivalence. In order to deal with states rather than weights, we perform a global Morita equivalence, obtained by reducing the groupoid $\mathcal{G}_{\mathbb{K}}$ by a suitable open set. The set $\mathbb{A}_{\mathbb{K}}^{(1)}$ of $(8.9)$ that we use to reduce the groupoid $\mathcal{G}_{\mathbb{K}}$ will only capture part of the classical subspace $C_{\mathbb{K}}$, but since our main focus is on the geometry of the complement of this subspace (the cokernel of the reduction map), this will not be a problem.

Lemma 8.4. Let $\mathbb{K}$ be a global field. Let $W \subset \mathbb{A}_{\mathbb{K}}$ be a neighborhood of $0 \in \mathbb{A}_{\mathbb{K}}$. Then for $x \in \mathbb{A}_{\mathbb{K}}$ one has $\mathbb{K}^{*} x \cap W \neq \emptyset$, unless $x \in \mathbb{A}_{\mathbb{K}}^{*}$ is an idele. For $x \in \mathbb{A}_{\mathbb{K}}^{*}$, the orbit $\mathbb{K}^{*} x$ is discrete in $\mathbb{A}_{\mathbb{K}}$. 
Proof. One can assume that $W$ is of the form

$$
W=\left\{a=\left(a_{w}\right)|| a_{w} \mid<\varepsilon \forall w \in S \text { and }\left|a_{w}\right| \leq 1 \forall w \notin S\right\},
$$

for $S$ a finite set of places and for some $\varepsilon>0$. Multiplying by a suitable idele one can in fact assume that $S=\emptyset$, so that we have

$$
W=\left\{a=\left(a_{w}\right)|| a_{w} \mid \leq 1 \forall w \in \Sigma_{\mathbb{K}}\right\} .
$$

One has $\left|x_{v}\right| \leq 1$ except on a finite set $F \subset \Sigma_{\mathbb{K}}$ of places. Moreover, if $x$ is not an idele, one can also assume that

$$
\prod_{v \in F}\left|x_{v}\right|<\delta
$$

for any fixed $\delta$. Thus, $-\log \left|x_{v}\right|$ is as large as one wants and there exists $k \in \mathbb{K}^{*}$ such that $k x \in W$. This is clear in the function field case because of the Riemann Roch formula (2.7). In the case of $\mathbb{Q}$ one can first multiply $x$ by an integer to get $\left|x_{v}\right| \leq 1$ for all finite places, then since this does not alter the product of all $\left|x_{v}\right|$ one gets $\left|x_{\infty}\right|<1$ and $x \in W$. The case of more general number fields is analogous. In the case of ideles, one can assume that $x=1$ and then the second statement follows from the discreteness of $\mathbb{K}$ in $\mathbb{A}_{\mathbb{K}}$.

We consider the following choice of a neighborhood of zero.

Definition 8.5. Consider the open neighborhood of $0 \in \mathbb{A}_{\mathbb{K}}$ defined by

$$
\mathbb{A}_{\mathbb{K}}^{(1)}=\prod_{w \in \Sigma_{\mathbb{K}}} \mathbb{K}_{w}^{(1)} \subset \mathbb{A}_{\mathbb{K}}
$$

where for any place we let $\mathbb{K}_{w}^{(1)}$ be the interior of $\left\{x \in \mathbb{K}_{w} ;|x| \leq 1\right\}$. Let $\mathcal{G}_{\mathbb{K}}^{(1)}$ denote the reduction of the groupoid $\mathcal{G}_{\mathbb{K}}$ by the open subset $\mathbb{A}_{\mathbb{K}}^{(1)} \subset \mathbb{A}_{\mathbb{K}}$ of the units and let $\mathcal{S}\left(\mathcal{G}_{\mathbb{K}}^{(1)}\right)$ denote the corresponding (smooth) groupoid algebra.

The algebra $\mathcal{S}\left(\mathcal{G}_{\mathbb{K}}^{(1)}\right)$ is a subalgebra of $\mathcal{S}\left(\mathcal{G}_{\mathbb{K}}\right)$ where one simply extends the function $f(k, x)$ by zero outside of the open subgroupoid $\mathcal{G}_{\mathbb{K}}^{(1)} \subset \mathcal{G}_{\mathbb{K}}$. With this convention, the convolution product of $\mathcal{S}\left(\mathcal{G}_{\mathbb{K}}^{(1)}\right)$ is simply given by the convolution product of $\mathcal{S}\left(\mathcal{G}_{\mathbb{K}}\right)$ of the form

$$
\left(f_{1} \star f_{2}\right)(k, x)=\sum_{h \in \mathbb{K}^{*}} f_{1}\left(k h^{-1}, h x\right) f_{2}(h, x) .
$$

We see from Lemma 8.4 above that the only effect of the reduction to $\mathcal{G}_{\mathbb{K}}^{(1)}$ is to remove from the noncommutative space $\mathbb{A}_{\mathbb{K}} / \mathbb{K}^{*}$ all the elements of $C_{\mathbb{K}}$ whose class modulo $\mathbb{K}^{*}$ does not intersect $\mathcal{G}_{\mathbb{K}}^{(1)}$ (i.e. in particular those whose norm is greater than or equal to one). We then have the following symmetries for the $\operatorname{algebra} \mathcal{S}\left(\mathcal{G}_{\mathbb{K}}^{(1)}\right)$. 
Proposition 8.6. Let $\mathcal{J}^{+}$denote the semi-group of ideles $j \in \mathbb{A}_{\mathbb{K}}^{*}$ such that $j \mathbb{A}_{\mathbb{K}}^{(1)} \subset \mathbb{A}_{\mathbb{K}}^{(1)}$. The semigroup $\mathcal{J}^{+}$acts on the algebra $\mathcal{S}\left(\mathcal{G}_{\mathbb{K}}^{(1)}\right)$ by endomorphisms obtained as restrictions of the automorphisms of $\mathcal{S}\left(\mathcal{G}_{\mathbb{K}}\right)$ of the form

$$
\vartheta_{a}(j)(f)(k, x)=f\left(k, j^{-1} x\right), \quad \forall(k, x) \in \mathcal{G}_{\mathbb{K}}, \quad j \in \mathcal{J}^{+} .
$$

Let $\mathbb{K}=\mathbb{Q}$ and $C_{\mathbb{Q}}^{+} \subset C_{\mathbb{Q}}$ be the semigroup $C_{\mathbb{Q}}^{+}=\left\{g \in C_{\mathbb{Q}}|| g \mid<1\right\}$. The semi-group $C_{\mathbb{Q}}^{+}$acts on $\mathcal{S}\left(\mathcal{G}_{\mathbb{Q}}^{(1)}\right)$ by the endomorphisms

$$
F(g)=\vartheta_{a}(\bar{g})
$$

with $\bar{g}$ the natural lift of $g \in C_{\mathbb{Q}}^{+}$to $\hat{\mathbb{Z}}^{*} \times \mathbb{R}_{+}^{*}$.

Proof. By construction $\vartheta_{a}(j)$ is an automorphism of $\mathcal{S}\left(\mathcal{G}_{\mathbb{K}}\right)$. For a function $f$ with support $B$ in the open set $\mathcal{G}_{\mathbb{K}}^{(1)}$ the support of the function $\vartheta_{a}(j)(f)$ is $j B=\{(k, j x) \mid(k, x) \in B\} \subset \mathcal{G}_{\mathbb{K}}^{(1)}$ so that $\vartheta_{a}(j)(f)$ still has support in $\mathcal{G}_{\mathbb{K}}^{(1)}$.

For $\mathbb{K}=\mathbb{Q}$ let $\bar{g} \in \hat{\mathbb{Z}}^{*} \times \mathbb{R}_{+}^{*}$ be the natural lift of an element $g \in C_{\mathbb{Q}}^{+}$. Then the archimedean component $\bar{g}_{\infty}$ is of absolute value less than 1 so that $\bar{g} \in \mathcal{J}^{+}$. The action of $\vartheta_{a}(\bar{g})$ by endomorphisms of $\mathcal{S}\left(\mathcal{G}_{\mathbb{Q}}^{(1)}\right)$ induces a corresponding action of $C_{\mathbb{Q}}^{+}$.

Remark 8.7. For $m$ a positive integer, consider the element $g=\left(1, m^{-1}\right) \in C_{\mathbb{Q}}^{+}$. Both $g=\left(1, m^{-1}\right)$ and $\tilde{m}=(m, 1)$ are in $\mathcal{J}^{+}$and have the same class in the idele class group $C_{\mathbb{Q}}$, since $m g=\tilde{m}$. Thus the automorphisms $\vartheta_{a}(g)$ and $\vartheta_{a}(\tilde{m})$ of $\mathcal{S}\left(\mathcal{G}_{\mathbb{K}}\right)$ are inner conjugate. Since the open set $\mathbb{A}_{\mathbb{K}}^{(1)} \subset \mathbb{A}_{\mathbb{K}}$ is not closed its characteristic function is not continuous and does not define a multiplier of $\mathcal{S}\left(\mathcal{G}_{\mathbb{K}}\right)$. It follows that the endomorphism $F(g)$ is inner conjugate to the endomorphism $\vartheta_{a}(\tilde{m})$ only in the following weaker sense. There exists a sequence of elements $u_{n}$ of $\mathcal{S}\left(\mathcal{G}_{\mathbb{K}}^{(1)}\right)$ such that for any $f \in \mathcal{S}\left(\mathcal{G}_{\mathbb{K}}^{(1)}\right)$ with compact support

$$
F(g)(f)=u_{n} \vartheta_{a}(\tilde{m})(f) u_{n}^{*},
$$

holds for all $n$ large enough.

8.2. The valuation systems. We now explain why the orbits $C_{\mathbb{K}} a^{(v)}$ appear indeed as the set of classical points, in the sense of the low temperature KMS states, of the noncommutative spaces $X_{\mathbb{K}, v}$. The notion of classical points obtained from low temperature KMS states is discussed at length in [15] ( $c f$. also [12, [13], 14]).

The noncommutative space $X_{\mathbb{K}, v}$ is described by the the restricted groupoid

$$
\mathcal{G}(v)=\mathbb{K}^{*} \ltimes \mathbb{A}_{\mathbb{K}, v}^{(1)}=\left\{(g, a) \in \mathbb{K}^{*} \ltimes \mathbb{A}_{\mathbb{K}, v} \mid a \text { and } g a \in \mathbb{A}_{\mathbb{K}, v}^{(1)}\right\} .
$$

We denote by $\varphi$ the positive functional on $C^{*}\left(\mathbb{K}^{*} \ltimes \mathbb{A}_{\mathbb{K}, v}^{(1)}\right)$ given by

$$
\varphi(f)=\int_{\mathbb{A}_{\mathbb{K}, v}^{(1)}} f(1, a) d a
$$


Proposition 8.8. The modular automorphism group of the functional $\varphi$ on the crossed product $C^{*}(\mathcal{G}(v))$ is given by the time evolution

$$
\sigma_{t}^{v}(f)(k, x)=|k|_{v}^{i t} f(k, x), \quad \forall t \in \mathbb{R}, \quad \forall f \in C^{*}\left(\mathbb{K}^{*} \ltimes \mathbb{A}_{\mathbb{K}, v}^{(1)}\right) .
$$

Proof. We identify elements of $C_{c}\left(\mathbb{K}^{*} \ltimes \mathbb{A}_{\mathbb{K}, v}^{(1)}\right)$ with functions $f(g, a)$ of elements $g \in \mathbb{K}^{*}$ and $a \in \mathbb{A}_{\mathbb{K}, v}^{(1)}$. The product is simply of the form

$$
f_{1} * f_{2}(g, a)=\sum_{r} f_{1}\left(g r^{-1}, g a\right) f_{2}(r, a) .
$$

The additive Haar measure $d a$ on $\mathbb{A}_{\mathbb{K}, v}$ satisfies the scaling property

$$
d(k a)=|k|_{v}^{-1} d a, \quad \forall k \in \mathbb{K}^{*},
$$

since the product measure $d a \times d a_{v}$ on $\mathbb{A}_{\mathbb{K}}=\mathbb{A}_{\mathbb{K}, v} \times \mathbb{K}_{v}$ is invariant under the scaling by $k \in \mathbb{K}^{*}$, while the additive Haar measure $d a_{v}$ on $\mathbb{K}_{v}$ gets multiplied by $|k|_{v}$, namely $d\left(k a_{v}\right)=|k|_{v} d a_{v}$. We then check the $\mathrm{KMS}_{1}$ condition, for $\varphi$ associated to the additive Haar measure, as follows,

$$
\begin{gathered}
\varphi\left(f_{1} * f_{2}\right)=\sum_{r} \int_{\mathbb{A}_{\mathbb{K}, v}^{(1)}} f_{1}\left(r^{-1}, r a\right) f_{2}(r, a) d a \\
=\sum_{r} \int_{\mathbb{A}_{\mathbb{K}, v}^{(1)}} f_{2}\left(k^{-1}, k b\right) f_{1}(k, b)|k|_{v}^{-1} d b=\varphi\left(f_{2} * \sigma_{i}\left(f_{1}\right)\right),
\end{gathered}
$$

using the change of variables $k=r^{-1}, a=k b$ and $d a=|k|_{v}^{-1} d b$.

It is worthwhile to observe that these automorphisms extend to the global algebra. Let $\mathcal{G}_{\mathbb{K}}^{(1)}$ be the groupoid $\mathbb{K}^{*} \ltimes \mathbb{A}_{\mathbb{K}}^{(1)}$ of Definition 8.5,

Lemma 8.9. Let $\mathbb{K}$ be a global field and $v \in \Sigma_{\mathbb{K}}$ a place. The map

$$
d_{v}(k, x)=\log |k|_{v} \in \mathbb{R}
$$

defines a homomorphism of the groupoid $\mathcal{G}_{\mathbb{K}}^{(1)}$ to the additive group $\mathbb{R}$ and the time evolution

$$
\sigma_{t}^{v}(f)(k, x)=|k|_{v}^{i t} f(k, x), \quad \forall t \in \mathbb{R}, \quad \forall f \in \mathcal{S}\left(\mathcal{G}_{\mathbb{K}}^{(1)}\right)
$$

generates a 1-parameter group of automorphisms of the algebra $\mathcal{S}\left(\mathcal{G}_{\mathbb{K}}^{(1)}\right)$.

The following result shows that the nontrivial part of the dynamics $\sigma_{t}^{v}$ concentrates on the algebra $\mathcal{S}(\mathcal{G}(v))$ with $\mathcal{G}(v)$ as in (8.12).

Proposition 8.10. The morphism $\rho_{v}$ of (8.6) restricts to a $\sigma_{t}^{v}$-equivariant morphism $\mathcal{S}\left(\mathcal{G}_{\mathbb{K}}^{(1)}\right) \rightarrow \mathcal{S}(\mathcal{G}(v))$. Moreover, the restriction of the one parameter group $\sigma_{t}^{v}$ to the kernel of $\rho_{v}$ is inner.

Proof. For the first statement note that the proper inclusion $\mathbb{A}_{\mathbb{K}, v} \subset \mathbb{A}_{\mathbb{K}}$ restricts to a proper inclusion $\mathbb{A}_{\mathbb{K}, v}^{(1)} \subset \mathbb{A}_{\mathbb{K}}^{(1)}$. For the second statement, notice that the formula

$$
h_{v}(x)=\log |x|_{v}, \quad \forall x \in \mathbb{A}_{\mathbb{K}}^{(1)},
$$


defines the multipliers $e^{i t h_{v}}$ of the kernel of $\rho_{v}$. Indeed $e^{i t h_{v}}$ is a bounded continuous function on $\mathbb{A}_{\mathbb{K}}^{(1)} \backslash \mathbb{A}_{\mathbb{K}, v}^{(1)}$.

We can then check that the 1-cocycle $d_{v}$ is the coboundary of $h_{v}$. In fact, we have

$$
h_{v}(k x)-h_{v}(x)=d_{v}(k, x), \quad \forall(k, x) \in \mathcal{G}_{\mathbb{K}}^{(1)} \backslash \mathcal{G}(v) .
$$

We now recall that, for an étale groupoid like $\mathcal{G}(v)$, every unit $y \in \mathcal{G}(v)^{(0)}$ defines, by

$$
\left(\pi_{y}(f) \xi\right)(\gamma)=\sum_{\gamma_{1} \gamma_{2}=\gamma} f\left(\gamma_{1}\right) \xi\left(\gamma_{2}\right)
$$

a representation $\pi_{y}$ by left convolution of the algebra of $\mathcal{G}(v)$ in the Hilbert space $\mathcal{H}_{y}=\ell^{2}\left(\mathcal{G}(v)_{y}\right)$, where $\mathcal{G}(v)_{y}$ denotes the set of elements of the groupoid $\mathcal{G}(v)$ with source $y$. By construction the unitary equivalence class of the representation $\pi_{y}$ is unaffected when one replaces $y$ by an equivalent $z \in \mathcal{G}(v)^{(0)}$ i.e. one assumes that there exists $\gamma \in \mathcal{G}(v)$ with range and source $y$ and $z$. Thus we can think of the label $y$ of $\pi_{y}$ as living in the quotient space $X_{\mathbb{K}, v}$ of equivalence classes of elements of $\mathcal{G}(v)^{(0)}$.

The relation between $\Xi_{\mathbb{K}, v}$ and $X_{\mathbb{K}, v}$ is then the following.

Theorem 8.11. For $y \in X_{\mathbb{K}, v}$, the representation $\pi_{y}$ is a positive energy representation if and only if $y \in \Xi_{\mathbb{K}, v}$.

Proof. Let first $y \in \mathcal{G}(v)^{(0)} \cap \Xi_{\mathbb{K}, v}$. Thus one has $y \in \mathbb{A}_{\mathbb{K}, v}^{(1)}, y_{w} \neq 0$ for all $w$ and $\left|y_{w}\right|=1$ for all $w \notin S$ where $S$ is a finite set of places. We can identify $\mathcal{G}(v)_{y}$ with the set of $k \in \mathbb{K}^{*}$ such that $k y \in \mathbb{A}_{\mathbb{K}, v}^{(1)}$. We extend $y$ to the adele $\tilde{y}=y \times 1$ whose component at the place $v$ is equal to $1 \in \mathbb{K}_{v}$. Then $\tilde{y}$ is an idele. Thus by Lemma 8.4 the number of elements of the orbit $\mathbb{K}^{*} \tilde{y}$ in a given compact subset of $\mathbb{A}_{\mathbb{K}}$ is finite. It follows that $\log |k|_{v}$ is lower bounded on $\mathcal{G}(v)_{y}$. Indeed otherwise there would exist a sequence $k_{n} \in \mathbb{K}^{*} \cap \mathcal{G}(v)_{y}$ such that $\left|k_{n}\right|_{v} \rightarrow 0$. Then $k_{n} \tilde{y} \in \mathbb{A}_{\mathbb{K}}^{(1)}$ for all $n$ large enough and this contradicts the discreteness of $\mathbb{K}^{*} \tilde{y}$. In the representation $\pi_{y}$ the time evolution $\sigma_{t}$ is implemented by the Hamiltonian $H_{y}$ given by

$$
\left(H_{y} \xi\right)(k, y)=\log |k|_{v} \xi(k, y)
$$

Namely, we have

$$
\pi_{y}\left(\sigma_{t}(f)\right)=e^{i t H_{y}} \pi_{y}(f) e^{-i t H_{y}}, \quad \forall f \in C_{c}(\mathcal{G}(v)) .
$$

Thus since $\log |k|_{v}$ is lower bounded on $\mathcal{G}(v)_{y}$ we get that the representation $\pi_{y}$ is a positive energy representation.

Let then $y \in \mathcal{G}(v)^{(0)} \backslash \Xi_{\mathbb{K}, v}$. We shall show that $\log |k|_{v}$ is not lower bounded on $\mathcal{G}(v)_{y}$, and thus that $\pi_{y}$ is not a positive energy representation. We consider as above the adele $\tilde{y}=y \times 1$ whose component at the place $v$ is equal to $1 \in \mathbb{K}_{v}$. 
Assume that $\log |k|_{v}$ is lower bounded on $\mathcal{G}(v)_{y}$. Then there exists $\epsilon>0$ such that, for $k \in \mathbb{K}^{*}$,

$$
k y \in \mathbb{A}_{\mathbb{K}, v}^{(1)} \Rightarrow|k|_{v} \geq \epsilon .
$$

This shows that the neighborhood of $0 \in \mathbb{A}_{\mathbb{K}}$ defined as

$$
W=\left\{a \in \mathbb{A}_{\mathbb{K}} ;\left|a_{v}\right|<\epsilon, a_{w} \in \mathbb{K}_{w}^{(1)}, \forall w \neq v\right\}
$$

does not intersect $\mathbb{K}^{*} \tilde{y}$. Thus by Lemma 8.4 we get that $\tilde{y}$ is an idele and $y \in$ $\Xi_{\mathbb{K}, v}$.

The specific example of the Bost-Connes system combined with Theorem 8.11 shows that one can refine the recipe of [15] ( $c f$. also [12, [13, [14]) for taking "classical points" of a noncommutative space. The latter recipe only provides a notion of classical points that can be thought of, by analogy with the positive characteristic case, as points defined over the mysterious "field with one element" $\mathbb{F}_{1}$ (see e.g. [28]). To obtain instead a viable notion of the points defined over the maximal unramified extension $\overline{\mathbb{F}}_{1}$, one performs the following sequence of operations.

$$
X \stackrel{\text { Dual System }}{\longrightarrow} \hat{X} \stackrel{\text { Periodic Orbits }}{\longrightarrow} \hat{X}_{v} \stackrel{\text { Classical Points }}{\longrightarrow} \cup \Xi_{v}
$$

which make sense in the framework of endomotives of 11. Note in particular that the dual system $\hat{X}$ is of type II and as such does not have a non-trivial time evolution. Thus it is only by restricting to the periodic orbits that one passes to noncommutative spaces of type III for which the cooling operation is non-trivial. In the analogy with geometry in non-zero characteristic, the set of points $X\left(\overline{\mathbb{F}}_{q}\right)$ over $\overline{\mathbb{F}}_{q}$ of a variety $X$ is indeed obtained as the union of the periodic orbits of the Frobenius.

Remark 8.12. Theorem 8.11 does not give the classification of $\mathrm{KMS}_{\beta}$ states for the quantum statistical system $\left(C^{*}\left(\mathbb{K}^{*} \ltimes \mathbb{A}_{\mathbb{K}, v}^{(1)}\right), \sigma_{t}\right)$. It just exhibits extremal $\mathrm{KMS}_{\beta}$ states but does not show that all of them are of this form.

8.3. The curve inside the adeles class space. In the case of a function field $\mathbb{K}=\mathbb{F}_{q}(C)$, the set of periodic classical points of the adeles class space $\mathbb{A}_{\mathbb{K}} / \mathbb{K}^{*}$ is (non-canonically) isomorphic to the algebraic points $C\left(\overline{\mathbb{F}}_{q}\right)$. In fact, more precisely the set of algebraic points $C\left(\overline{\mathbb{F}}_{q}\right)$ is equivariantly isomorphic to the quotient $\Xi_{\mathbb{K}} / C_{\mathbb{K}, 1}$ where $C_{\mathbb{K}, 1} \subset C_{\mathbb{K}}$ is the kernel of the norm $|\cdot|: C_{\mathbb{K}} \rightarrow \mathbb{R}_{+}^{*}$, and $\Xi_{\mathbb{K}}$ is as in (8.8).

Proposition 8.13. For $\mathbb{K}=\mathbb{F}_{q}(C)$ a function field, the orbits of Frobenius on $C\left(\overline{\mathbb{F}}_{q}\right)$ give an equivariant identification

$$
\Xi_{\mathbb{K}} / C_{\mathbb{K}, 1} \simeq C\left(\overline{\mathbb{F}}_{q}\right),
$$

between $\Xi_{\mathbb{K}} / C_{\mathbb{K}, 1}$ with the action of $q^{\mathbb{Z}}$ and $C\left(\overline{\mathbb{F}}_{q}\right)$ with the action of the group of integer powers of the Frobenius. 
Proof. At each place $v \in \Sigma_{\mathbb{K}}$ the quotient group of the range $N$ of the norm $|\cdot|: C_{\mathbb{K}} \rightarrow \mathbb{R}_{+}^{*}$ by the range $N_{v}$ of $|\cdot|: \mathbb{K}_{v} \rightarrow \mathbb{R}_{+}^{*}$ is the finite cyclic group

$$
N / N_{v}=q^{\mathbb{Z}} / q^{n_{v} \mathbb{Z}} \simeq \mathbb{Z} / n_{v} \mathbb{Z},
$$

where $n_{v}$ is the degree of the place $v \in \Sigma_{\mathbb{K}}$. The degree $n_{v}$ is the same as the cardinality of the orbit of the Frobenius acting on the fiber of the map (2.2) from algebraic points in $C\left(\overline{\mathbb{F}}_{q}\right)$ to places in $\Sigma_{\mathbb{K}}$. Thus, one can construct in this way an equivariant embedding

$$
C\left(\overline{\mathbb{F}}_{q}\right) \hookrightarrow\left(\mathbb{A}_{\mathbb{K}} / \mathbb{K}^{*}\right) / C_{\mathbb{K}, 1}
$$

obtained, after choosing a point in each orbit, by mapping the orbit of the integer powers of the Frobenius in $C\left(\overline{\mathbb{F}}_{q}\right)$ over a place $v$ to the orbit of $C_{\mathbb{K}} / C_{\mathbb{K}, 1} \sim q^{\mathbb{Z}}$ on the adele $a^{(v)}$.

Modulo the problem created by the fact that the identification above is noncanonical and relies upon the choice of a point in each orbit, it is then possible to think of the locus $\Xi_{\mathbb{K}}$, in the number field case, as a replacement for $C\left(\overline{\mathbb{F}}_{q}\right)$ inside the adeles class space $\mathbb{A}_{\mathbb{K}} / \mathbb{K}^{*}$.

In the case of $\mathbb{K}=\mathbb{Q}$, the quotient $\Xi_{\mathbb{Q}} / C_{\mathbb{Q}, 1}$ appears as a union of periodic orbits of period $\log p$ under the action of $C_{\mathbb{Q}} / C_{\mathbb{Q}, 1} \sim \mathbb{R}$, as in Figure 1 . What matters, however, is not the space $\Xi_{\mathbb{Q}} / C_{\mathbb{Q}, 1}$ in itself but the way it sits inside $\mathbb{A}_{\mathbb{Q}} / \mathbb{Q}^{*}$. Without taking into account the topology induced by $\mathbb{A}_{\mathbb{K}}$ the space $\Xi_{\mathbb{K}}$ would just be a disjoint union of orbits without any interesting global structure, while it is the embedding in the adeles class space that provides the geometric setting underlying the Lefschetz trace formula of [10] and its cohomological formulation of [11].

8.4. The valuation systems for $\mathbb{K}=\mathbb{Q}$. We concentrate again on the specific case of $\mathbb{K}=\mathbb{Q}$ to understand better the properties of the dynamical systems $\sigma_{t}^{p}$ associated to the finite primes $p \in \Sigma_{\mathbb{Q}}$.

We know that, in the case of the $\mathrm{BC}$ system, the KMS state at critical temperature $\beta=1$ is given by the additive Haar measure on finite adeles [5]. Thus, one expects that, for the systems associated to the finite primes, the additive Haar measure of $\mathbb{A}_{\mathbb{Q}, p}$ should play an analogous role.

Definition 8.14. Let $\mathbb{A}_{\mathbb{Q}, p}^{*} \subset \mathbb{A}_{\mathbb{Q}, p}^{(1)}$ be the subspace

$$
\mathbb{A}_{\mathbb{Q}, p}^{*}=\left\{x \in \mathbb{A}_{\mathbb{Q}, p}|| x_{w} \mid=1 \forall w \neq p, \infty \text { and } p^{-1} \leq\left|x_{\infty}\right|<1\right\} .
$$

As above $\mathcal{G}(p)$ denotes the reduction of the groupoid $\mathcal{G}_{\mathbb{Q}, p}$ by the open subset $\mathbb{A}_{\mathbb{Q}, p}^{(1)} \subset \mathbb{A}_{\mathbb{Q}, p}$, namely

$$
\mathcal{G}(p)=\left\{(k, x) \in \mathcal{G}_{\mathbb{Q}, p} \mid x \in \mathbb{A}_{\mathbb{Q}, p}^{(1)}, k x \in \mathbb{A}_{\mathbb{Q}, p}^{(1)}\right\} .
$$

Notice that the set $\mathbb{A}_{\mathbb{Q}, p}^{(1)}$ meets all the equivalence classes in $\mathbb{A}_{\mathbb{Q}, p}$ by the action of $\mathbb{Q}^{*}$. In fact, given $x \in \mathbb{A}_{\mathbb{Q}, p}$, one can find a representative $y$ with $y \sim x$ in 


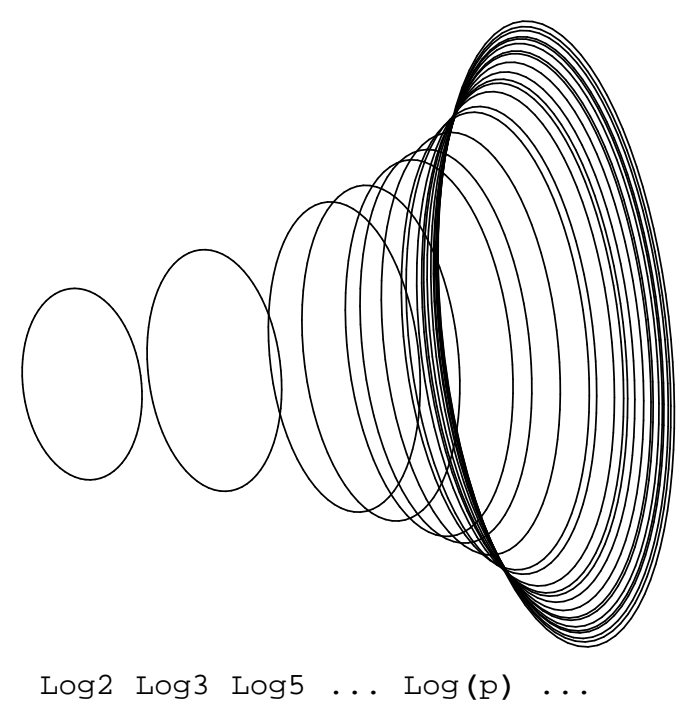

Figure 1 . The classical points $\Xi_{\mathbb{Q}} / C_{\mathbb{Q}, 1}$ of the adeles class space $\mathbb{A}_{\mathbb{Q}} / \mathbb{Q}^{*}$.

$\mathbb{A}_{\mathbb{Q}, p} / \mathbb{Q}^{*}$, such that $y \in \hat{\mathbb{Z}} \times \mathbb{R}$. Upon multiplying $y$ by a suitable power of $p$, one can make $y_{\infty}$ as small as required, and in particular one can obtain in this way a representative in $\mathbb{A}_{\mathbb{Q}, p}^{(1)}$. Let us assume that $\left|y_{w}\right|=1$ for all finite places $w \neq p$ and that $y_{\infty}>0$. Then there exists a unique $n \in \mathbb{N} \cup\{0\}$ such that $p^{n} y \in \mathbb{A}_{\mathbb{Q}, p}^{*}$. Given a prime $p$ we define the function $f_{p}(\lambda, \beta)$ for $\lambda \in(1, p]$ and $\beta>1$ by

$$
f_{p}(\lambda, \beta)=\sum c_{k} p^{-k \beta}
$$

where the $c_{k} \in\{0, \ldots p-1\}$ are the digits of the expansion of $\lambda$ in base $p$. There is an ambiguous case where all digits $c_{k}$ are equal to 0 for $k>m$ while $c_{m}>0$, since the same number

$$
\lambda=\sum c_{k} p^{-k}
$$

is obtained using the same $c_{j}$ for $j<m, c_{m}-1$ instead of $c_{m}$ and $c_{j}=p-1$ for $j>m$. In that case, for $\beta>1$, (8.29) gives two different values and we choose the value coming from the second representation of $\lambda$, i.e. the lower of the two. These coefficients $c_{k}$ of the expansion of $\lambda$ in base $p$ are then given by

$$
c_{k}=\left\lceil\lambda p^{k}-1\right\rceil-p\left\lceil\lambda p^{k-1}-1\right\rceil,
$$

where $\lceil x\rceil=\inf _{n \in \mathbb{Z}}\{n \geq x\}$ denotes the ceiling function.

Note that, for $\beta>1$, the function $f_{p}(\lambda, \beta)$ is discontinuous ( $c f$. Figures 2 and 3) at any point $(\lambda, \beta)$ where the expansion of $\lambda$ in base $p$ is ambiguous, i.e. $\lambda \in \mathbb{N} p^{-k}$. 


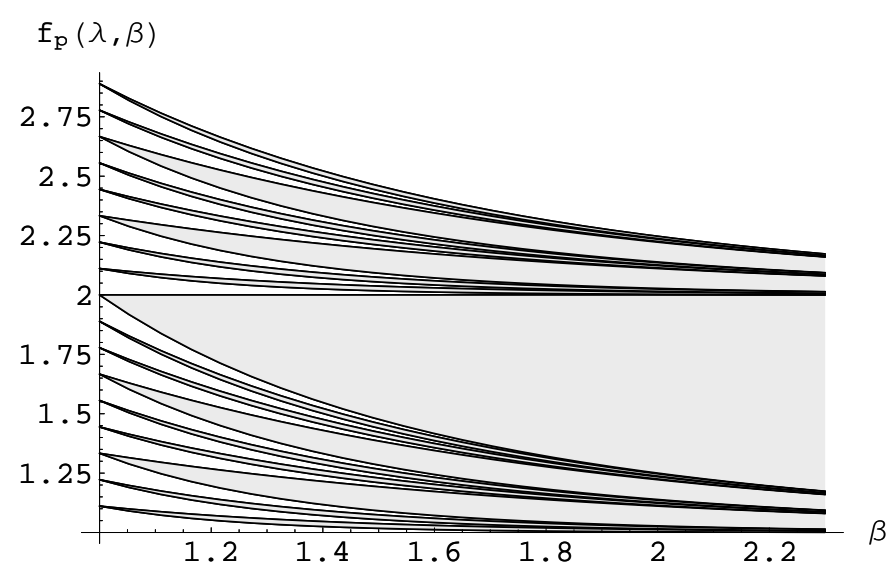

Figure 2. Graphs of the functions $f_{p}(\lambda, \beta)$ as functions of $\beta$ for $p=3, \lambda=n / 27$. The gray regions are the gaps in the range of $f_{p}$.

Moreover for $\beta=1$ one gets

$$
f_{p}(\lambda, 1)=\lambda, \quad \forall \lambda \in(1, p] .
$$

We then obtain the following result.

Theorem 8.15. Let $\left(C^{*}(\mathcal{G}(p)), \sigma_{t}^{p}\right)$ be the $C^{*}$-dynamical system associated to the groupoid (8.28) with the time evolution (8.17). Then the following properties hold:

(1) For any $y \in \mathbb{A}_{\mathbb{Q}, p}^{*}$ the corresponding representation $\pi_{y}$ has positive energy.

(2) Let $H_{y}$ denote the Hamiltonian implementing the time evolution in the representation $\pi_{y}$, for $y \in \mathbb{A}_{\mathbb{Q}, p}^{*}$ with $y_{\infty}=\lambda^{-1}$ and $\lambda \in(1, p]$. Then the partition function is given by

$$
Z_{p}(\lambda, \beta)=\operatorname{Tr}\left(e^{-\beta H_{y}}\right)=2 \frac{1-p^{-\beta}}{1-p^{1-\beta}} f_{p}(\lambda, \beta) .
$$

(3) The functionals

$$
\psi_{\beta, y}(a)=\operatorname{Tr}\left(e^{-\beta H_{y}} \pi_{y}(a)\right), \quad \forall a \in C^{*}(\mathcal{G}(p))
$$

satisfy the $K M S_{\beta}$ condition for $\sigma_{t}^{p}$ and depend weakly continuously on the parameter $y \in \mathbb{A}_{\mathbb{Q}, p}^{*}$.

Proof. (1) This follows from Theorem 8.11, For $y \in \mathbb{A}_{\mathbb{Q}, p}^{*}$ one has

$$
r \in \mathbb{Q}^{*}, r y \in \mathbb{A}_{\mathbb{Q}, p}^{(1)} \Longrightarrow r=p^{-k} m,
$$

for some $k \geq 0$ and some integer $m$ prime to $p$ and such that $\left|r y_{\infty}\right|<1$. This implies

$$
|m|<p^{k+1}
$$


and one finds

$$
|r|_{p}=p^{k} \geq 1 \quad \text { and } \quad \log |r|_{p} \geq 0
$$

In fact, the argument above shows that the spectrum of the Hamiltonian $H_{y}$ implementing the time evolution $\sigma_{t}^{p}$ in the representation $\pi_{y}$ is given by

$$
\operatorname{Spec}\left(H_{y}\right)=\{k \log p\}_{k \in \mathbb{N} \cup\{0\}},
$$

hence $\pi_{y}$ is a positive energy representation.

(2) We begin by the special case with $y_{\infty}=p^{-1}$. Then $\lambda=p$ and $f_{p}(\lambda, \beta)=\frac{p-1}{1-p^{-\beta}}$ since all digits of $\lambda=p$ are equal to $p-1$. We want to show that the partition function is given by

$$
\operatorname{Tr}\left(e^{-\beta H_{y}}\right)=2 \frac{p-1}{1-p^{1-\beta}} .
$$

The multiplicity of an eigenvalue $k \log p$ of $H_{y}$ is the number of integers $m \neq 0 \in \mathbb{Z}$ that are prime to $p$ and such that $p^{-k}|m| y_{\infty}<1$. Since we are assuming that $y_{\infty}=p^{-1}$, this gives $|m|<p^{k+1}$. Thus, the multiplicity is just $2\left(p^{k+1}-p^{k}\right)$. The factor 2 comes from the sign of the integer $m$. The factor $\left(p^{k+1}-p^{k}\right)$ corresponds to subtracting from the number $p^{k+1}$ of positive integers $m \leq p^{k+1}$ the number $p^{k}$ of those that are multiples of $p$.

We now pass to the general case. For $x>0,\lceil x-1\rceil$ is the cardinality of $(0, x) \cap \mathbb{N}$. The same argument used above shows that the multiplicity of the eigenvalue $k \log p$ is given by the counting

$$
2\left(\left\lceil\lambda p^{k}-1\right\rceil-\left\lceil\lambda p^{k-1}-1\right\rceil\right) .
$$

Thus

$$
\operatorname{Tr}\left(e^{-\beta H_{y}}\right)=2 \sum_{k=0}^{\infty}\left(\left\lceil\lambda p^{k}-1\right\rceil-\left\lceil\lambda p^{k-1}-1\right\rceil\right) p^{-k \beta} .
$$

One has the following equalities of convergent series,

$$
\sum_{k=0}^{\infty}\left(\left\lceil\lambda p^{k}-1\right\rceil-\left\lceil\lambda p^{k-1}-1\right\rceil\right) p^{-k \beta}=\sum_{k=0}^{\infty}\left\lceil\lambda p^{k}-1\right\rceil\left(p^{-k \beta}-p^{-(k+1) \beta}\right)
$$

so that,

$$
\operatorname{Tr}\left(e^{-\beta H_{y}}\right)=2\left(1-p^{-\beta}\right) \sum_{k=0}^{\infty}\left\lceil\lambda p^{k}-1\right\rceil p^{-k \beta}
$$

Similarly

$$
\sum_{k=0}^{\infty}\left(\left\lceil\lambda p^{k}-1\right\rceil-p\left\lceil\lambda p^{k-1}-1\right\rceil\right) p^{-k \beta}=\sum_{k=0}^{\infty}\left\lceil\lambda p^{k}-1\right\rceil\left(p^{-k \beta}-p p^{-(k+1) \beta}\right)
$$

which gives

$$
f_{p}(\lambda, \beta)=\left(1-p^{1-\beta}\right) \sum_{k=0}^{\infty}\left\lceil\lambda p^{k}-1\right\rceil p^{-k \beta}
$$




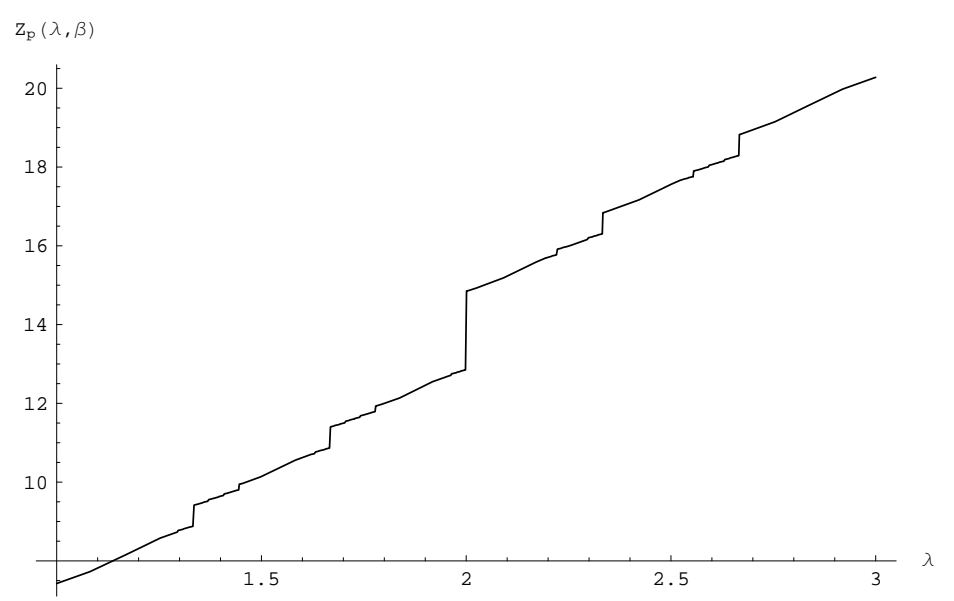

Figure 3 . Graph of the function $Z_{p}(\lambda, \beta)$ as a function of $\lambda$ for $p=3, \beta=1.2$.

since the coefficients $c_{k}$ of the expansion of $\lambda$ in base $p$ are given by (8.30). Combining (8.40) with (8.41) gives (8.32).

(3) It follows from (8.22) and the finiteness of the partition function (8.32) that the functionals (8.33) fulfill the $\mathrm{KMS}_{\beta}$ condition. In terms of functions on the groupoid $\mathcal{G}(p)$ one has

$$
\psi_{\beta, y}(f)=\sum f\left(1, n p^{-k} y\right) p^{-k \beta}, \quad \forall f \in C_{c}(\mathcal{G}(p))
$$

where the sum is absolutely convergent. Each of the terms in the sum gives a weakly continuous linear form thus one obtains the required continuity.

Remark 8.16. The partition function $Z_{p}(\lambda, \beta)$ is a discontinuous function of the parameter $\lambda$ and this might seem to contradict the third statement of Theorem 8.15. It would if the algebra $C^{*}(\mathcal{G}(p))$ were unital since, in that case, the partition function is given by evaluation on the unit and weak continuity implies that it is continuous. In our case $C^{*}(\mathcal{G}(p))$ is not unital, and the partition function is expressed as a supremum of the form

$$
Z_{p}(\lambda, \beta)=\sup \left\{\psi_{\beta, y}\left(a^{*} a\right) \mid a \in C^{*}(\mathcal{G}(p)),\|a\| \leq 1\right\} .
$$

In particular it shows that $Z_{p}(\lambda, \beta)$ is lower semi-continuous as a function of $\lambda$.

The precise qualitative properties of the partition functions $Z_{p}(\lambda, \beta)$ are described by the following result

Proposition 8.17. As a function of $\lambda \in(1, \lambda]$ the partition function $Z_{p}(\lambda, \beta)$ satisfies for $\beta>1$ :

(1) $Z_{p}$ is strictly increasing.

(2) $Z_{p}$ is continuous on the left, and lower semi-continuous. 
(3) $Z_{p}$ is discontinuous at any point of the form $\lambda=m p^{-k}$ with a jump of $2 p^{-k \beta}$ (for $m$ prime to $p$ ).

(4) The measure $\frac{\partial Z_{p}}{\partial \lambda}$ is the sum of the Dirac masses at the points $\lambda=m p^{-k}$, $m$ prime to $p$, with coefficients $2 p^{-k \beta}$.

(5) The closure of the range of $Z_{p}$ is a Cantor set.

Proof. (1) This follows from (8.40) which expresses $Z_{p}$ as an absolutely convergent sum of multiples of the functions $\left[\lambda p^{k}-1\right\rceil$. The latter are non-decreasing and jump by 1 at $\lambda \in \mathbb{N} p^{-k} \cap(1, p]$. The density of the union of these finite sets for $k \geq 0$ shows that $Z_{p}$ is strictly increasing.

(2) This follows as above from (8.40) and the semi-continuity properties of the ceiling function.

(3) Let $\lambda=m p^{-k}$ with $m$ prime to $p$. Then for any $j \geq k$ one gets a jump of $2\left(1-p^{-\beta}\right) p^{-j \beta}$ coming from (8.40) so that their sum gives

$$
2\left(1-p^{-\beta}\right) \sum_{j=k}^{\infty} p^{-j \beta}=2 p^{-k \beta}
$$

(4) This follows as above from (8.40) and from (3) which computes the discontinuity at the jumps.

(5) Recall that when writing elements of an interval in base $p$ one gets a map from the cantor set to the interval. This map is surjective but fails to be injective due to the identifications coming from $\sum_{0}^{\infty}(p-1) p^{-m}=p$. The connectedness of the interval is recovered from these identifications. In our case the coefficients $c_{k}$ of the expansion in base $p$ of elements of $(1, p]$ are such that $c_{0} \in\{1, \ldots, p-1\}$ while $c_{k} \in\{0, \ldots, p-1\}$ for $k>0$. This is a Cantor set $K$ in the product topology of $K=\{1, \ldots, p-1\} \times \prod_{\mathbb{N}}\{0, \ldots, p-1\}$. As shown in Figure 3, the discontinuities of the function $Z_{p}(\lambda, \beta)$ as a function of $\lambda$ replace the connected topology of $(1, p]$ by the totally disconnected topology of $K$.

Remark 8.18. One can use (8.39) to define $Z_{p}(\lambda, \beta)$ for any $\lambda>0$, as

$$
Z_{p}(\lambda, \beta)=2 \sum_{-\infty}^{\infty}\left(\left\lceil\lambda p^{k}-1\right\rceil-\left\lceil\lambda p^{k-1}-1\right\rceil\right) p^{-k \beta}
$$

This makes sense for $\Re(\beta)>1$ since $\left\lceil\lambda p^{k}-1\right\rceil=0$ for $k \leq-\frac{\log \lambda}{\log p}$. The extended function (8.43) satisfies

$$
Z_{p}(p \lambda, \beta)=p^{\beta} Z_{p}(\lambda, \beta)
$$

which suggests replacing $Z_{p}(\lambda, \beta)$ with

$$
\zeta_{p}(\lambda, \beta)=\lambda^{-\beta} Z_{p}(\lambda, \beta)
$$

so that

$$
\zeta_{p}(p \lambda, \beta)=\zeta_{p}(\lambda, \beta)
$$




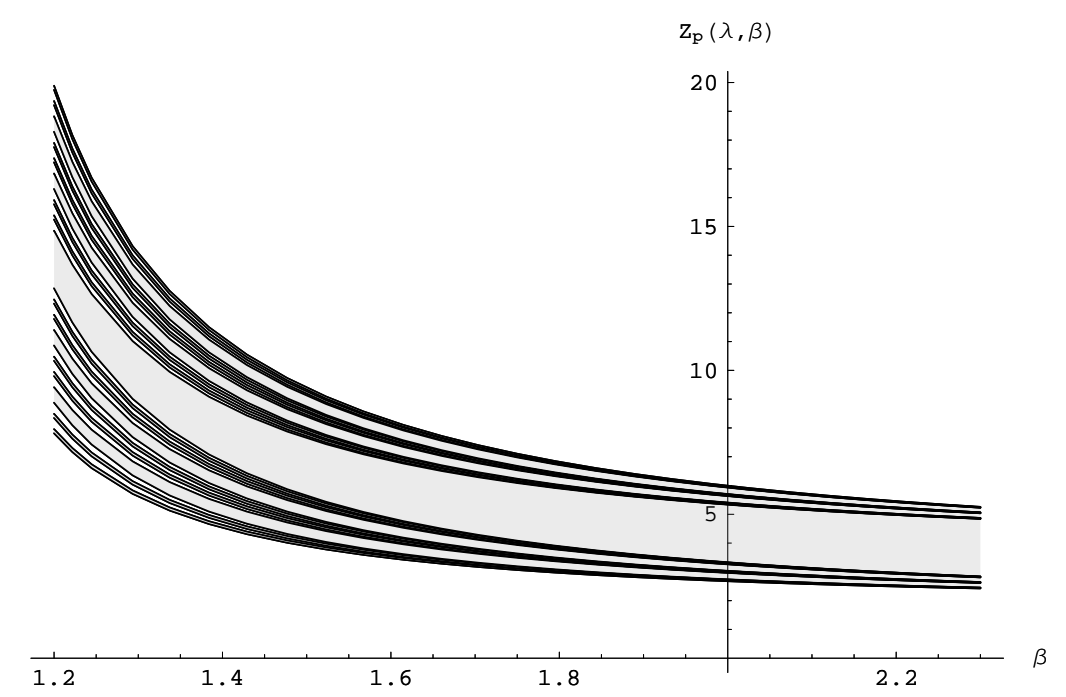

Figure 4 . Graphs of the functions $Z_{p}(\lambda, \beta)$ as functions of $\beta$ for $p=3, \lambda=n / 27$. The gray regions are the gaps in the range. All these functions have a pole at $\beta=1$.

This replacement $Z_{p} \mapsto \zeta_{p}$ corresponds to the shift in the Hamiltonian $H_{y}$ by

$$
H_{y} \mapsto H_{y}-\log \left|y_{\infty}\right| .
$$

We can now refine Theorem 8.11 and consider the zero temperature KMS state of the system $\left(C^{*}(\mathcal{G}(p)), \sigma_{t}^{p}\right)$ corresponding to the positive energy representation $\pi_{y}$ for $y \in \Xi_{\mathbb{Q}, p}$.

Proposition 8.19. As $\beta \rightarrow \infty$ the vacuum states (zero temperature KMS states) of the system $\left(C^{*}(\mathcal{G}(p)), \sigma_{t}^{p}\right)$ with Hamiltonian $H_{y}$ have a degeneracy of $2\lceil\lambda-1\rceil$, where $y_{\infty}=\lambda^{-1}$. There is a preferred choice of a vacuum state given by the evaluation at $y \in \mathbb{A}_{\mathbb{Q}, p}^{*}$.

Proof. When we look at the orbit of $y \in \mathbb{A}_{\mathbb{Q}, p}^{*}$, i.e. at the intersection $\mathbb{Q}^{*} y \cap \mathbb{A}_{\mathbb{Q}, p}^{(1)}$ and label its elements by pairs $(k, m)$ as above, we find that all elements with $k=0$ give a ground state. This degeneracy of the vacuum reflects the fact that the limit of the partition function as the temperature goes to 0 is not in general equal to 1 . For instance, for $y_{\infty}=p^{-1}$, one finds

$$
\lim _{\beta \rightarrow \infty} \operatorname{Tr}\left(e^{-\beta H_{y}}\right)=\lim _{\beta \rightarrow \infty} 2 \frac{p-1}{1-p^{1-\beta}}=2(p-1) .
$$

More generally, one finds similarly the limit

$$
\lim _{\beta \rightarrow \infty} \operatorname{Tr}\left(e^{-\beta H_{y}}\right)=2\lceil\lambda-1\rceil .
$$

Among the $2\lceil\lambda-1\rceil$ vacuum states, the state given by evaluation at $y \in \mathbb{A}_{\mathbb{Q}, p}^{*}$ is singled out, since $m y \notin \mathbb{A}_{\mathbb{Q}, p}^{*}$ for $m \neq 1$. It is then natural to consider, for each 
finite place $p \in \Sigma_{\mathbb{Q}}$, the section

$$
s_{p}(x)=\mathbb{Q}^{*} x \cap \mathbb{A}_{\mathbb{Q}, p}^{*}, \quad \forall x \in C_{\mathbb{Q}} a^{(p)} \subset \mathbb{A}_{\mathbb{Q}} / \mathbb{Q}^{*}
$$

of the projection from $\mathbb{A}_{\mathbb{Q}}$ to the orbit $C_{\mathbb{Q}} a^{(p)}$.

Notice that $s_{p}$ is discontinuous at the boundary of the domain $\mathbb{A}_{\mathbb{Q}, p}^{*}$. Indeed when $y_{\infty}$ crosses the value $p^{-1}$ the class in $C_{\mathbb{Q}} a^{(p)}$ varies continuously but the representative in $\mathbb{A}_{\mathbb{Q}, p}^{*}$ jumps discontinuously so that its archimedian component remains in the interval $\left[p^{-1}, 1\right)$. This suggests to consider a cyclic covering of $\Xi_{\mathbb{Q}}$ which we now discuss in 88.5 .

8.5. The cyclic covering $\tilde{\Xi}_{\mathbb{Q}}$ of $\Xi_{\mathbb{Q}}$. By construction $\Xi_{\mathbb{K}}$ is a subspace of the adeles class space $X_{\mathbb{K}}$. We shall now show, in the case $\mathbb{K}=\mathbb{Q}$, that it admits a natural lift $\tilde{\Xi}_{\mathbb{Q}}$ to a subspace of $\mathbb{A}_{\mathbb{Q}}$ which reduces the ambiguity group $\mathbb{Q}^{*}$ to a cyclic group. One thus obtains a natural cyclic covering $\tilde{\Xi}_{\mathbb{Q}} \subset \mathbb{A}_{\mathbb{Q}}$ of $\Xi_{\mathbb{Q}}$. We already saw above, in Proposition 8.19, that it is natural to choose representatives for the elements of the orbit $C_{\mathbb{Q}} a^{(p)}$, for a finite prime $p$, in the subset of adeles given by

$$
\tilde{\Xi}_{\mathbb{Q}, p}:=\left\{y \in \mathbb{A}_{\mathbb{Q}} \mid y_{p}=0 \text { and }\left|y_{\ell}\right|=1 \text { for } \ell \neq p, \infty \text { and } y_{\infty}>0\right\} .
$$

We extend this definition at $\infty$ by

$$
\tilde{\Xi}_{\mathbb{Q}, \infty}:=\left\{y \in \mathbb{A}_{\mathbb{Q}}|| y_{w} \mid=1 \forall w \neq \infty \text { and } y_{\infty}=0\right\} .
$$

Definition 8.20. The locus $\tilde{\Xi}_{\mathbb{Q}} \subset \mathbb{A}_{\mathbb{Q}}$ is defined as

$$
\tilde{\Xi}_{\mathbb{Q}}=\bigcup_{v \in \Sigma_{\mathbb{Q}}} \tilde{\Xi}_{\mathbb{Q}, v} \subset \mathbb{A}_{\mathbb{Q}}
$$

We then have the following simple fact.

Proposition 8.21. Let $\pi$ be the projection from $\tilde{\Xi}_{\mathbb{Q}}$ to $\Xi_{\mathbb{Q}}$, with $\pi(x)$ the class of $x$ modulo the action of $\mathbb{Q}^{*}$.

(1) The map $\pi: \tilde{\Xi}_{\mathbb{Q}} \rightarrow \Xi_{\mathbb{Q}}$ is surjective.

(2) Two elements in $\tilde{\Xi}_{\mathbb{Q}, v}$ have the same image in $C_{\mathbb{Q}} a^{(v)}$ iff they are on the same orbit of the following transformation $T$

$$
T x=p x, \quad \forall x \in \tilde{\Xi}_{\mathbb{Q}, p}, \quad T x=-x, \quad \forall x \in \tilde{\Xi}_{\mathbb{Q}, \infty}
$$

Proof. The first statement follows by lifting $C_{\mathbb{Q}}$ inside $\mathbb{A}_{\mathbb{Q}}^{*}$ as the subgroup $\hat{\mathbb{Z}}^{*} \times$ $\mathbb{R}_{+}^{*}$. Then any element of $C_{\mathbb{Q}} a^{(v)}$ has a representative in $\left(\hat{\mathbb{Z}}^{*} \times \mathbb{R}_{+}^{*}\right) a^{(v)}$

The proof of the second statement is straightforward, since for a finite prime $p$ the subgroup $p^{\mathbb{Z}} \subset \mathbb{Q}^{*}$ is the group of elements of $\mathbb{Q}^{*}$ which leave $\tilde{\Xi}_{\mathbb{Q}, p}$ globally invariant. 
8.6. Arithmetic subalgebra, Frobenius and monodromy. We now describe a natural algebra of coordinates $\mathcal{B}$ on $\Xi_{\mathbb{Q}}$.

The BC system of [5], as well as its arithmetic generalizations of [12] and [14], have the important property that they come endowed with an arithmetic structure given by an arithmetic subalgebra. The general framework of endomotives developed in [11] shows a broad class of examples where a similar arithmetic structure is naturally present. We consider here the issue of extending the construction of the "rational subalgebra" of the BC-system to the algebra $\mathcal{S}\left(\mathcal{G}_{\mathbb{Q}}^{(1)}\right)$ of 丹8.1.

In order to get a good geometric picture it is convenient to think in terms of $\mathbb{Q}$-lattices rather than of adeles, as in $[12]$. Thus, we let $\mathcal{L}$ denote the set of 1-dimensional $\mathbb{Q}$-lattices (as defined in [12]). We consider the map

$$
\iota: \hat{\mathbb{Z}} \times \mathbb{R}_{+}^{*} \rightarrow \mathcal{L}, \quad \iota(\rho, \lambda)=(\Lambda, \phi)=\left(\lambda^{-1} \mathbb{Z}, \lambda^{-1} \rho\right)
$$

which associates to an adele $(\rho, \lambda) \in \hat{\mathbb{Z}} \times \mathbb{R}_{+}^{*} \subset \mathbb{A}_{\mathbb{Q}}$ the $\mathbb{Q}$-lattice obtained using $\rho$ to label the torsion points of $\mathbb{R} / \lambda^{-1} \mathbb{Z}$. Replacing $(\rho, \lambda)$ by $(n \rho, n \lambda)$, for a positive integer $n \in \mathbb{N}$, one obtains the pair $\left(\frac{1}{n} \Lambda, \phi\right)$, which is commensurable to $(\Lambda, \phi)$. Thus, the action of $\mathbb{Q}_{+}^{*}$ corresponds to commensurability of $\mathbb{Q}$-lattices under the map $\iota$. Multiplying $\lambda$ by a positive scalar corresponds to the scaling action of $\mathbb{R}_{+}^{*}$ on $\mathbb{Q}$-lattices.

Let us recall the definition of the "rational algebra" $\mathcal{A}_{\mathbb{Q}}$ of $[12]$ for the BC system, given in terms of $\mathbb{Q}$-lattices. We let

$$
\epsilon_{a}(\Lambda, \phi)=\sum_{y \in \Lambda+\phi(a)} y^{-1}
$$

for any $a \in \mathbb{Q} / \mathbb{Z}$. This is well defined, for $\phi(a) \neq 0$, using the summation $\lim _{N \rightarrow \infty} \sum_{-N}^{N}$, and is zero by definition for $\phi(a)=0$. The function

$$
\varphi_{a}(\rho, \lambda)=\epsilon_{a}(\iota(\rho, \lambda)), \quad \forall(\rho, \lambda) \in \hat{\mathbb{Z}} \times \mathbb{R}_{+}^{*},
$$

is well defined and homogeneous of degree 1 in $\lambda$. Moreover, for fixed $a \in \mathbb{Q} / \mathbb{Z}$ with denominator $m$, it only depends upon the projection of $\rho$ on the finite group $\mathbb{Z} / m \mathbb{Z}$, hence it defines a continuous function on $\hat{\mathbb{Z}} \times \mathbb{R}_{+}^{*}$. Using the degree 1 homogeneity in $\lambda$, one gets that (8.53) extends by continuity to 0 on $\hat{\mathbb{Z}} \times\{0\}$. One gets functions that are homogeneous of weight zero by taking the derivatives of the functions $\varphi_{a}$. The functions

$$
\psi_{a}(\rho, \lambda)=\frac{1}{2 \pi i} \frac{d}{d \lambda} \varphi_{a}(\rho, \lambda), \quad \forall(\rho, \lambda) \in \hat{\mathbb{Z}} \times \mathbb{R}_{+}^{*},
$$

are independent of $\lambda$ hence they define continuous functions on $\mathbb{A}_{\mathbb{Q}}^{(1)}$. They are non trivial on $\tilde{\Xi}_{\mathbb{Q}, \infty}=\hat{\mathbb{Z}}^{*} \times\{0\} \subset \hat{\mathbb{Z}} \times\{0\}$ and they agree there with the functions $e_{a}$ of [12].

Proposition 8.22. Let $\mathcal{B}$ be the algebra generated by the $\varphi_{a}$ and $\psi_{a}$ defined in (8.53) and (8.54) above. 
(1) The expression

$$
N(f)=\frac{1}{2 \pi i} \frac{d}{d \lambda} f
$$

defines a derivation $N$ of $\mathcal{B}$.

(2) The algebra $\mathcal{B}$ is stable under the derivation $Y$ that generates the 1 parameter semigroup $F(\mu)$ of endomorphisms of $\mathcal{S}\left(\mathcal{G}_{\mathbb{Q}}^{(1)}\right)$ of (8.11) and one has, at the formal level, the relation

$$
F(\mu) N=\mu N F(\mu) .
$$

(3) For any element $f \in \mathcal{B}$ one has

$$
\alpha \circ f(x)=f(\tilde{\alpha} x), \quad \forall x \in \tilde{\Xi}_{\mathbb{Q}, \infty} \text { and } \forall \alpha \in \operatorname{Gal}\left(\mathbb{Q}^{c y c l} / \mathbb{Q}\right),
$$

where $\tilde{\alpha} \in \hat{\mathbb{Z}}^{*} \subset C_{\mathbb{Q}}$ is the element of the idele class group associated to $\alpha \in \operatorname{Gal}\left(\mathbb{Q}^{\text {cycl }} / \mathbb{Q}\right)$ by the class field theory isomorphism.

Proof. 1) By construction $N$ is a derivation of the algebra of functions. Moreover (8.54) shows that $N\left(\varphi_{a}\right)=\psi_{a}$, while $N\left(\psi_{a}\right)=0$. Thus, the derivation rule shows that $\mathcal{B}$ is stable under $N$.

2) The derivation generating the one parameter semigroup $F(\mu)$ is given, up to sign, by the grading operator

$$
Y(f)=\lambda \frac{d}{d \lambda} f
$$

By construction, any of the $\varphi_{a}$ is of degree one, i.e. $Y\left(\varphi_{a}\right)=\varphi_{a}$ and $\psi_{a}$ is of degree 0 . Thus, again the derivation rule shows that $\mathcal{B}$ is stable under $Y$.

3) This only involves the functions $\psi_{a}$, since by construction the restriction of $\varphi_{a}$ is zero on $\tilde{\Xi}_{\mathbb{Q}, \infty}$. The result then follows from the main result of [5] in the reformulation given in [12] (see also [13], Chapter 3). In fact, all these functions take values in the cyclotomic field $\mathbb{Q}^{\text {cycl }} \subset \mathbb{C}$ and they intertwine the action of the discontinuous piece $\hat{\mathbb{Z}}^{*}$ of $C_{\mathbb{Q}}$ with the action of the Galois group of $\mathbb{Q}^{\text {cycl }}$.

This is in agreement with viewing the algebra $\mathcal{B}$ as the algebra of coordinates on $\tilde{\Xi}_{\mathbb{O}}$. Indeed, in the case of a global field $\mathbb{K}$ of positive characteristic, the action of the Frobenius on the points of $C\left(\overline{\mathbb{F}}_{q}\right)$ (which have coordinates in $\overline{\mathbb{F}}_{q}$ ) corresponds to the Frobenius map

$$
\text { Fr }: u \mapsto u^{q}, \quad \forall u \in \mathbb{K}
$$

of the function field $\mathbb{K}$ of the curve $C$. The Frobenius endomorphism $u \mapsto u^{q}$ of $\mathbb{K}$ is the operation that replaces a function $f: C\left(\overline{\mathbb{F}}_{q}\right) \rightarrow \overline{\mathbb{F}}_{q}$ by its $q$-th power, i.e. the composition Fr $\circ f$ with the Frobenius automorphism $\operatorname{Fr} \in \operatorname{Gal}\left(\overline{\mathbb{F}}_{q} / \mathbb{F}_{q}\right)$. For $f \in \mathbb{K}$, one has

$$
\text { Fr } \circ f=f^{q}=f \circ \text { Fr, }
$$

where on the right hand side Fr is the map that raises every coordinate to the power $q$. This corresponds to the interwtining with the Galois action discussed above. 
Notice moreover that, as we have seen in Proposition 8.6, only the semigroup $C_{\mathbb{Q}}^{+}$

acts on the reduced system $\mathcal{S}\left(\mathcal{G}_{\mathbb{Q}}^{(1)}\right)$ and it acts by endomorphisms. It nevertheless acts in a bijective manner on the points of $\Xi_{\mathbb{Q}}$. This is similar to what happens with the Frobenius endomorphism (8.59), which is only an endomorphism of the field of functions $\overline{\mathbb{K}}$, while it acts bijectively (as a Galois automorphism of the coordinates) on the points of $C\left(\overline{\mathbb{F}}_{q}\right)$.

Further notice that there is a striking formal analogy between the operators $F$ and $N$ of Proposition 8.22 satisfying the relation (8.56) and the Frobenius and local monodromy operators introduced in the context of the "special fiber at arithmetic infinity" in Arakelov geometry (see [18, [19]). In particular, one should compare (8.56) with $\S 2.5$ of [19] that discusses a notion of Weil-Deligne group at arithmetic infinity.

\section{Functoriality of the Adeles Class space}

We investigate in this section the functoriality of the adele class space $X_{\mathbb{K}}$ and of its classical subspace $\Xi_{\mathbb{K}} \subset X_{\mathbb{K}}$, for Galois extensions of the global field $\mathbb{K}$.

This issue is related to the question of functoriality. Namely, given a finite algebraic extension $\mathbb{L}$ of the global field $\mathbb{K}$, we want to relate the adele class spaces of both fields. Assume the extension is a Galois extension. In general, we do not expect the relation between the adeles class spaces to be canonical, in the sense that it will involve a symmetry breaking choice on the Galois group $G=\operatorname{Gal}(\mathbb{L} / \mathbb{K})$ of the extension. More precisely, the norm map

$$
\mathfrak{n}(a)=\prod_{\sigma \in G} \sigma(a) \in \mathbb{A}_{\mathbb{K}}, \quad \forall a \in \mathbb{A}_{\mathbb{L}}
$$

appears to be the obvious candidate that relates the two adeles class spaces. In fact, since $\mathfrak{n}(\mathbb{L}) \subset \mathbb{K}$, the map (9.1) passes to the quotient and gives a natural map from $X_{\mathbb{L}}=\mathbb{A}_{\mathbb{L}} / \mathbb{L}^{*}$ to $X_{\mathbb{K}}=\mathbb{A}_{\mathbb{K}} / \mathbb{K}^{*}$ that looks like the covering required by functoriality. However, the problem is that the norm map fails to be surjective in general, hence it certainly does not qualify as a covering map. In fact, this problem already occurs at the level of the idele class group $C_{\mathbb{K}}$, namely the norm map fails to be a surjection from $C_{\mathbb{L}}$ to $C_{\mathbb{K}}$.

The correct object to consider is the Weil group $W_{\mathbb{L}, \mathbb{K}}$. This is an extension of $C_{\mathbb{L}}$ by the Galois group $G=\operatorname{Gal}(\mathbb{L} / \mathbb{K})$, which is not a semi-direct product. The corresponding non-trivial 2-cocycle is called the "fundamental class". One has a natural morphism $t$, called the transfer, from $W_{\mathbb{L}, \mathbb{K}}$ to $C_{\mathbb{K}}$. The transfer satisfies the following two properties.

- The morphism $t$ restricts to the norm map from $C_{\mathbb{L}}$ to $C_{\mathbb{K}}$.

- The morphism $t$ is surjective on $C_{\mathbb{K}}$

Thus, the correct way to understand the relation between the adeles class spaces $X_{\mathbb{L}}$ and $X_{\mathbb{K}}$ is by extending the construction of the Weil group and of the the transfer map. 
One obtains in this way $n$ copies of the adele class space $X_{\mathbb{L}}$ of $\mathbb{L}$ and a map to $X_{\mathbb{K}}$ which is now a covering from $G \times \Xi_{\mathbb{L}} \rightarrow \Xi_{\mathbb{K}}$. This space has a natural action of the Weil group. We explain this in more detail in what follows.

9.1. The norm map. We begin by recalling the well known properties of the norm map that are relevant to our set-up. Thus, we let $\mathbb{L} \supset \mathbb{K}$ be a finite Galois extension of $\mathbb{K}$ of degree $n$, with $G=\operatorname{Gal}(\mathbb{L} / \mathbb{K})$ the Galois group.

Since the adeles depend naturally on the field, one has a canonical action of $G$ on $\mathbb{A}_{\mathbb{L}}$. If $v \in \Sigma_{\mathbb{K}}$ is a place of $\mathbb{K}$, there are $m_{v}$ places of $\mathbb{L}$ over $v$ and they are permuted transitively by the action of $G$. Let $G_{w}$ be the isotropy subgroup of $w$. Then $G_{w}$ is the Galois group $G_{w}=\operatorname{Gal}\left(\mathbb{L}_{w} / \mathbb{K}_{v}\right)$.

One has a canonical embedding of $\mathbb{A}_{\mathbb{K}}$ as the fixed points of the action of $G$ on $\mathbb{A}_{\mathbb{L}}$ by

$$
\mathbb{A}_{\mathbb{K}}=\mathbb{A}_{\mathbb{L}}^{G}, \quad\left(a_{v}\right) \mapsto\left(a_{\pi(w)}\right), \quad \text { with } \quad \pi: \Sigma_{\mathbb{L}} \rightarrow \Sigma_{\mathbb{K}}
$$

The norm map $\mathfrak{n}: \mathbb{A}_{\mathbb{L}} \rightarrow \mathbb{A}_{\mathbb{K}}$ is then defined as in (9.1). By [43] IV 1, Corollary 3 , it is given explicitly by

$$
\mathfrak{n}(x)=z, \quad z_{v}=\prod_{w \mid v} \mathfrak{n}_{\mathbb{L}_{w} / \mathbb{K}_{v}}\left(x_{w}\right), \quad \forall x \in \mathbb{A}_{\mathbb{L}} .
$$

Here the notation $w \mid v$ means that $w$ is a place of $\mathbb{L}$ over the place $v \in \Sigma_{\mathbb{K}}$. Also $\mathfrak{n}_{\mathbb{L}_{w} / \mathbb{K}_{v}}$ is the norm map of the extension $\mathbb{L}_{w} / \mathbb{K}_{v}$. When restricted to principal adeles of $\mathbb{L}$ it gives the norm map from $\mathbb{L}$ to $\mathbb{K}$. When restricted to the subgroup $\mathbb{L}_{w}^{*}=(\ldots, 1, \ldots, y, \ldots, 1, \ldots) \subset \mathbb{A}_{\mathbb{L}}^{*}$, it gives the norm map of the extension $\mathbb{L}_{w} / \mathbb{K}_{v}$. For nontrivial extensions this map is never surjective, but its restriction $\mathfrak{n}: \mathcal{O}\left(\mathbb{L}_{w}\right)^{*} \rightarrow \mathcal{O}\left(\mathbb{K}_{v}\right)^{*}$ is surjective when the extension is unramified, which is the case for almost all places $v \in \Sigma_{\mathbb{K}}(c f$. [43], Theorem 1 p.153). In such cases, the module of the subgroup $\mathfrak{n}\left(\mathbb{L}_{w}^{*}\right) \subset \mathbb{K}_{v}^{*}$ is a subgroup of index the order of the extension $\mathbb{K}_{v} \subset \mathbb{L}_{w}$. The restriction of the norm map to the idele group $\mathbb{A}_{\mathbb{L}}^{*}$ is very far from surjective to $\mathbb{A}_{\mathbb{K}}^{*}$ and its range is a subgroup of infinite index. The situation is much better with the idele class groups since ( $c f$. 43], Corollary p.153) the norm map is an open mapping $\mathfrak{n}: C_{\mathbb{L}} \rightarrow C_{\mathbb{K}}$ whose range is a subgroup of finite index.

9.2. The Weil group and the transfer map. The Weil group $W_{\mathbb{L}, \mathbb{K}}$ associated to the Galois extension $\mathbb{K} \subset \mathbb{L}$ is an extension

$$
1 \rightarrow C_{\mathbb{L}} \rightarrow W_{\mathbb{L}, \mathbb{K}} \rightarrow G \rightarrow 1
$$

of $C_{\mathbb{L}}$ by the Galois group $G$. One chooses a section $s$ from $G$ and lets $a \in$ $Z^{2}\left(G, C_{\mathbb{L}}\right)$ be the corresponding 2-cocycle so that

$$
a_{\alpha, \beta}=s_{\alpha \beta}^{-1} s_{\alpha} s_{\beta}, \quad \forall \alpha, \beta \in G .
$$

The algebraic rules in $W_{\mathbb{L}, \mathbb{K}}$ are then given by

$$
s_{\alpha} s_{\beta}=s_{\alpha \beta} a_{\alpha, \beta}, \quad \forall \alpha, \beta \in G
$$

and

$$
s_{\alpha} x s_{\alpha}^{-1}=\alpha(x), \quad \forall \alpha \in G, \quad \forall x \in C_{\mathbb{L}} .
$$


The transfer homomorphism

$$
t: W_{\mathbb{L}, \mathbb{K}} \rightarrow C_{\mathbb{K}}
$$

is then given by

$$
t(x)=\mathfrak{n}(x), \quad \forall x \in C_{\mathbb{L}} \quad \text { and } \quad t\left(s_{\alpha}\right)=\prod_{\beta} a_{\alpha, \beta}, \quad \forall \alpha \in G .
$$

Its main properties are the following (see [39]).

- $t$ is a surjective group morphism $W_{\mathbb{L}, \mathbb{K}} \rightarrow C_{\mathbb{K}}$.

- Let $W_{\mathbb{L}, \mathbb{K}}^{a b}$ be the abelian quotient of $W_{\mathbb{L}, \mathbb{K}}$ by the closure of its commutator subgroup $W_{\mathbb{L}, \mathbb{K}}^{c}$. Then $t$ induces an isomorphism of $W_{\mathbb{L}, \mathbb{K}}^{a b}$ with $C_{\mathbb{K}}$.

9.3. The covering. We finally describe the resulting functoriality of the adeles class spaces in terms of a covering map obtained by extending the Weil group and transfer map described above. Let, as above, $\mathbb{L} \supset \mathbb{K}$ be a finite Galois extension of $\mathbb{K}$.

Lemma 9.1. The transfer map extends to a map

$$
\tau: G \times X_{\mathbb{L}} \rightarrow X_{\mathbb{K}}
$$

of the adele class spaces.

Proof. We endow $G \times X_{\mathbb{L}}$ with a two sided action of $G$ compatible with $\tau$. By construction the norm map $\mathfrak{n}$ of (9.1) is well defined on $\mathbb{A}_{\mathbb{L}}$. Since it is multiplicative and we have $\mathfrak{n}\left(\mathbb{L}^{*}\right) \subset \mathbb{K}^{*}$, it induces a map of quotient spaces $\mathfrak{n}: X_{\mathbb{L}} \rightarrow X_{\mathbb{K}}$. By construction $C_{\mathbb{L}}$ acts on $X_{\mathbb{L}}$ and the actions by left and right multiplication coincide, so we use both notations. We define the map $\tau$ as

$$
\tau: G \times X_{\mathbb{L}} \rightarrow X_{\mathbb{K}}, \quad \tau(\alpha, x)=t\left(s_{\alpha}\right) \mathfrak{n}(x), \quad \forall x \in X_{\mathbb{L}}, \forall \alpha \in G .
$$

This makes sense since $t\left(s_{\alpha}\right) \in C_{\mathbb{K}}$ and $C_{\mathbb{K}}$ acts on $X_{\mathbb{K}}$. By construction, the restriction of $\tau$ to $G \times C_{\mathbb{L}}$ is the transfer map.

One identifies $G \times C_{\mathbb{L}}$ with $W_{\mathbb{L}, \mathbb{K}}$ by the map which to $(\alpha, g) \in G \times C_{\mathbb{L}}$ associates the element $s_{\alpha} g$ of $W_{\mathbb{L}, \mathbb{K}}$.

In the following we use the notation

$$
x^{g}=g^{-1}(x) .
$$

We have the following result.

Lemma 9.2. Let $\mathbb{L} \supset \mathbb{K}$ be a finite Galois extension of $\mathbb{K}$.

(1) The expressions

$$
s_{\alpha} g(\beta, x)=\left(\alpha \beta, a_{\alpha, \beta} g^{\beta} x\right), \quad \text { and } \quad(\alpha, x) s_{\beta} g=\left(\alpha \beta, a_{\alpha, \beta} x^{\beta} g\right)
$$

define a left and a right action of $W_{\mathbb{L}, \mathbb{K}}$ on $G \times X_{\mathbb{L}}$.

(2) The map $\tau$ of (9.11) satisfies the equivariance property

$$
\tau(g x k)=t(g) \tau(x) t(k), \quad \forall x \in G \times X_{\mathbb{L}}, \quad \text { and } \quad \forall g, k \in W_{\mathbb{L}, \mathbb{K}} .
$$


Proof. 1) We defined the rules (9.6) as the natural extension of the multiplication in $W_{\mathbb{L}, \mathbb{K}}$ using

$$
s_{\alpha} g s_{\beta} h=s_{\alpha} s_{\beta} g^{\beta} h=s_{\alpha \beta} a_{\alpha, \beta} g^{\beta} h .
$$

Thus, the proof of associativity in the group $W_{\mathbb{L}, \mathbb{K}}$ extends and it implies that (9.13) defines a left and a right action of $W_{\mathbb{L}, \mathbb{K}}$ and that these two actions commute.

2) The proof that the transfer map $t$ is a group homomorphism extends to give the required equality, since the norm map is a bimodule morphism when extended to $X_{\mathbb{L}}$.

At the level of the classical points, we can then describe the covering map in the following way.

Proposition 9.3. Let $\mathbb{L}$ and $\mathbb{K}$ be as above.

(1) The restriction of $\tau$ to $G \times \Xi_{\mathbb{L}} \subset G \times X_{\mathbb{L}}$ defines a surjection

$$
\tau: G \times \Xi_{\mathbb{L}} \rightarrow \Xi_{\mathbb{K}}
$$

(2) The map $\tau$ induces a surjection

$$
\tau: G \times\left(\Xi_{\mathbb{L}} / C_{\mathbb{L}, 1}\right) \rightarrow \Xi_{\mathbb{K}} / C_{\mathbb{K}, 1} .
$$

Proof. 1) By construction $\Xi_{\mathbb{L}}=\cup_{w \in \Sigma_{\mathbb{L}}} C_{\mathbb{L}} a^{(w)}$, where $a^{(w)} \in X_{\mathbb{L}}$ is the class, modulo the action of $\mathbb{L}^{*}$, of the adele with all entries equal to 1 except for a zero at $w$ as in (8.7). Let $\pi$ denote the natural surjection from $\Sigma_{\mathbb{L}}$ to $\Sigma_{\mathbb{K}}$. One has

$$
\tau\left(1, a^{(w)}\right)=a^{(\pi(w))}, \quad \forall w \in \Sigma_{\mathbb{L}} .
$$

In fact, one has $\tau\left(1, a^{(w)}\right)=\mathfrak{n}\left(a^{(w)}\right)$. Moreover, by (9.3), the adele $a=\mathfrak{n}\left(a^{(w)}\right)$ has components $a_{z}=1$ for all $z \neq \pi(w)$ and $a_{\pi(w)}=0$. Thus $a=a^{(\pi(w))}$. The equivariance of the map $\tau$ as in Lemma 9.2 together with the surjectivity of the transfer map from $W_{\mathbb{L}, \mathbb{K}}$ to $C_{\mathbb{K}}$ then show that we have

$$
\tau\left(W_{\mathbb{L}, \mathbb{K}}\left(1, a^{(w)}\right)\right)=C_{\mathbb{K}} a^{(\pi(w))}, \quad \forall w \in \Sigma_{\mathbb{L}} .
$$

For $s_{\alpha} g \in W_{\mathbb{L}, \mathbb{K}}$, one has

$$
s_{\alpha} g\left(1, a^{(w)}\right)=\left(\alpha, g a^{(w)}\right),
$$

since $a_{\alpha, 1}=1$. Thus, $W_{\mathbb{L}, \mathbb{K}}\left(1, a^{(w)}\right)=G \times C_{\mathbb{L}} a^{(w)}$ and one gets

$$
\tau\left(G \times C_{\mathbb{L}} a^{(w)}\right)=C_{\mathbb{K}} a^{(\pi(w))}, \quad \forall w \in \Sigma_{\mathbb{L}} .
$$

Since the map $\pi$ is surjective we get the conclusion.

2) The transfer map satisfies $t\left(C_{\mathbb{L}, 1}\right) \subset C_{\mathbb{K}, 1}$. When restricted to the subgroup $C_{\mathbb{L}}$ the transfer coincides with the norm map $\mathfrak{n}$ and in particular if $|g|=1$ one has $|\mathfrak{n}(g)|=1$. Thus one obtains a surjection of the quotient spaces

$$
\tau:\left(G \times \Xi_{\mathbb{L}}\right) / C_{\mathbb{L}, 1} \rightarrow \Xi_{\mathbb{K}} / C_{\mathbb{K}, 1}
$$

Moreover, the right action of the subgroup $C_{\mathbb{L}, 1} \subset W_{\mathbb{L}, \mathbb{K}}$ is given by

$$
(\alpha, x) g=(\alpha, x g) \text {. }
$$


This means that we can identify

$$
\left(G \times \Xi_{\mathbb{L}}\right) / C_{\mathbb{L}, 1} \sim G \times\left(\Xi_{\mathbb{L}} / C_{\mathbb{L}, 1}\right)
$$

9.4. The function field case. Let $\mathbb{K}=\mathbb{F}_{q}(C)$ be a global field of positive characteristic, identified with the field of rational functions on a nonsingular curve $C$ over $\mathbb{F}_{q}$. We consider the extensions

$$
\mathbb{L}=\mathbb{K} \otimes_{\mathbb{F}_{q}} \mathbb{F}_{q^{n}}
$$

The Galois group $G$ is the cyclic group of order $n$ with generator $\sigma \in \operatorname{Gal}(\mathbb{L} / \mathbb{K})$ given by $\sigma=\mathrm{id} \otimes \mathrm{Fr}$, where $\operatorname{Fr} \in \operatorname{Gal}\left(\mathbb{F}_{q^{n}} / \mathbb{F}_{q}\right)$ is the Frobenius automorphism. Given a point $x \in C\left(\overline{\mathbb{F}}_{q}\right)$ we let $n$ be the order of its orbit under the Frobenius. One then has $x \in C\left(\mathbb{F}_{q^{n}}\right)$ and evaluation at $x$ gives a well defined place $w(x) \in \Sigma_{\mathbb{L}}$. The projection $\pi(w(x)) \in \Sigma_{\mathbb{K}}$ is a well defined place of $\mathbb{K}$ which is invariant under $x \mapsto \operatorname{Fr}(x)$.

In the isomorphism of $\mathbb{Z}$-spaces

$$
\vartheta_{\mathbb{L}}: C\left(\overline{\mathbb{F}}_{q}\right) \rightarrow \Xi_{\mathbb{L}} / C_{\mathbb{L}, 1}
$$

described in 8 , we have no ambiguity for places corresponding to points $x \in$ $C\left(\mathbb{F}_{q^{n}}\right)$. To such a point we assign simply

$$
\vartheta_{\mathbb{L}}(x)=a^{(w(x))} \in \Xi_{\mathbb{L}} / C_{\mathbb{L}, 1} .
$$

We now describe what happens with these points of $\Xi_{\mathbb{L}} / C_{\mathbb{L}, 1}$ under the covering map $\tau$. We first need to see explicitly why the surjectivity only occurs after crossing by $G$.

Proposition 9.4. Let $\mathbb{K}$ and $\mathbb{L}=\mathbb{K} \otimes_{\mathbb{F}_{q}} \mathbb{F}_{q^{n}}$ be as above.

(1) The image $\mathfrak{n}\left(C_{\mathbb{L}}\right) \subset C_{\mathbb{K}}$ is the kernel of the morphism from $C_{\mathbb{K}}$ to $G=$ $\mathbb{Z} / n \mathbb{Z}$ given by

$$
g \mapsto \rho(g)=\log _{q}|g| \bmod n .
$$

(2) One has $\rho\left(t\left(s_{\sigma}\right)\right)=1 \bmod n$, where $\sigma \in \operatorname{Gal}(\mathbb{L} / \mathbb{K})$ is the Frobenius generator.

Proof. Since $\mathbb{L}$ is an abelian extension of $\mathbb{K}$, one has the inclusions

$$
\mathbb{K} \subset \mathbb{L} \subset \mathbb{K}^{a b} \subset \mathbb{L}^{a b},
$$

where $\mathbb{K}^{a b}$ is the maximal abelian extension of $\mathbb{K}$. Using the class field theory isomorphisms

$$
C_{\mathbb{K}} \sim W\left(\mathbb{K}^{a b} / \mathbb{K}\right) \quad \text { and } \quad C_{\mathbb{L}} \sim W\left(\mathbb{L}^{a b} / \mathbb{L}\right),
$$

one can translate the proposition in terms of Galois groups. The result then follows using [39] p.502. 


\section{VANISHING CYCLES: AN ANALOGY}

We begin by considering some simple examples that illustrate some aspects of the geometry of the adeles class space, by restricting to the semilocal case of a finite number of places. This will also illustrate more explicitly the idea of considering the adeles class space as a noncommutative compactification of the idele class group.

We draw an analogy between the complement of the idele classes in the adele classes and the singular fiber of a degeneration. This analogy should be taken with a big grain of salt, since this complement is a highly singular space and it really makes sense only as a noncommutative space in the motivic sense described in sections 4 and 5 above.

10.1. Two real places. We first consider the example of the real quadratic field $\mathbb{K}=\mathbb{Q}(\sqrt{2})$ and we restrict to its two real places $v_{1}$ and $v_{2}$. Thus, we replace the adeles $\mathbb{A}_{\mathbb{K}}$ simply by the product $\mathbb{K}_{v_{1}} \times \mathbb{K}_{v_{2}}$ over the real places, which is just the product of two copies of $\mathbb{R}$. The ideles $\mathbb{A}_{\mathbb{K}}^{*}$ are correspondingly replaced by $\mathbb{K}_{v_{1}}^{*} \times \mathbb{K}_{v_{2}}^{*}$ and the inclusion of ideles in adeles is simply given by the inclusion

$$
\left(\mathbb{R}^{*}\right)^{2} \subset \mathbb{R}^{2}
$$

The role of the action of the group $\mathbb{K}^{*}$ by multiplication is now replaced by the action by multiplication of the group $U$ of units of $\mathbb{K}=\mathbb{Q}(\sqrt{2})$. This group is

$$
U=\mathbb{Z} / 2 \mathbb{Z} \times \mathbb{Z}
$$

where the $\mathbb{Z} / 2 \mathbb{Z}$ comes from \pm 1 and the $\mathbb{Z}$ is generated by the unit $u=3-2 \sqrt{2}$. Its action on $\mathbb{R}^{2}$ is given by the transformation

$$
S(x, y)=\left(u x, u^{-1} y\right) .
$$

Thus, in this case of two real places the semi-local version of the adeles class space is the quotient

$$
X_{v_{1}, v_{2}}:=\mathbb{R}^{2} / U
$$

of $\mathbb{R}^{2}$ by the symmetry $(x, y) \mapsto(-x,-y)$ and the transformation $S$.

Both of these transformations preserve the function

$$
\tilde{f}: \mathbb{R}^{2} \rightarrow \mathbb{R}, \quad \tilde{f}(x, y)=x y,
$$

which descends to a function

$$
f: X_{v_{1}, v_{2}} \rightarrow \mathbb{R}
$$

Moreover one has

$$
(x, y) \in\left(\mathbb{R}^{*}\right)^{2} \subset \mathbb{R}^{2} \Leftrightarrow f(x, y) \neq 0
$$

and the fiber of $f$ over any non zero $\varepsilon \in \mathbb{R}$ is easily identified with a one dimensional torus

$$
f^{-1}(\varepsilon) \sim \mathbb{R}_{+}^{*} / u^{\mathbb{Z}}, \quad \forall \varepsilon \neq 0
$$

where one can use the map $(x, y) \mapsto|x|$ to obtain the required isomorphism. 
The fiber $f^{-1}(0)$ of $f$ over the point $\varepsilon=0$, on the other hand, is no longer a one dimensional torus and it is singular. It is the union of three pieces

$$
f^{-1}(0)=T_{1} \cup T_{2} \cup\{0\}
$$

corresponding respectively to

- $T_{1}$ is the locus $x=0, y \neq 0$, which is a torus $T_{1} \sim \mathbb{R}_{+}^{*} / u^{\mathbb{Z}}$ under the identification given by the map $(x, y) \mapsto|y|$.

- $T_{2}$ is the locus $x \neq 0, y=0$, which is also identified with a torus $T_{2} \sim$ $\mathbb{R}_{+}^{*} / u^{\mathbb{Z}}$ under the analogous map $(x, y) \mapsto|x|$.

- The last piece is the single point $x=0, y=0$.

One can see that at the naive level that the quotient topology on the singular fiber (10.7) looks as follows. For any point $x \in T_{j}$ its closure is $\bar{x}=\{x\} \cup\{0\}$. Moreover the point 0 is closed and the induced topology on its complement is the same as the disjoint union of two one dimensional tori $T_{j}$. In fact one can be more precise and see what happens by analyzing the $C^{*}$-algebras involved. The $C^{*}$-algebra $A$ associated to the singular fiber is by construction the crossed product

$$
A=C_{0}\left(\tilde{f}^{-1}(0)\right) \rtimes U
$$

with $\tilde{f}$ as in (10.4). One lets

$$
A_{j}=C_{0}\left(V_{j}\right) \rtimes U
$$

where we use the restriction of the action of $U$ to the subsets

$$
V_{j}=\left\{\left(x_{1}, x_{2}\right) \mid x_{j}=0\right\} \sim \mathbb{R} .
$$

Evaluation at $0 \in \mathbb{R}$ gives a homomorphism

$$
\epsilon_{j}: A_{j} \rightarrow C^{*}(U)
$$

Lemma 10.1. One has an exact sequence of the form

$$
0 \rightarrow C\left(T_{j}\right) \otimes \mathcal{K} \rightarrow A_{j} \stackrel{\epsilon_{j}}{\rightarrow} C^{*}(U) \rightarrow 0,
$$

where $\mathcal{K}$ is the algebra of compact operators.

The $C^{*}$-algebra $A$ is the fibered product of the $A_{j}$ over $C^{*}(U)$ using the morphisms $\epsilon_{j}$.

Proof. The first statement follows using the fact that the action of $U$ on $\mathbb{R}^{*}$ is free. Since the decomposition of $\tilde{f}^{-1}(0)$ as the union of the $V_{j}$ over their common point 0 is $U$-equivariant one gets the second statement.

After collapsing the spectrum of $C^{*}(U)$ to a point, the topology of the spectrum of $A_{j}$ is the topology of $T_{j} \cup\{0\}$ described above. The topology of the spectrum of $A$ is the topology of $f^{-1}(0)$ of (10.7) described above. 
10.2. A real and a non-archimedean place. We now consider another example, namely the case of $\mathbb{K}=\mathbb{Q}$ with two places $v_{1}, v_{2}$, where $v_{1}=p$ is a non-archimedean place associated to a prime $p$ and $v_{2}=\infty$ is the real place. Again, we replace adeles by the product $\mathbb{K}_{v_{1}} \times \mathbb{K}_{v_{2}}$ over the two places, which in this case is just the product

$$
\mathbb{K}_{v_{1}} \times \mathbb{K}_{v_{2}}=\mathbb{Q}_{p} \times \mathbb{R} .
$$

The ideles are correspondingly replaced by $\mathbb{K}_{v_{1}}^{*} \times \mathbb{K}_{v_{2}}^{*}=\mathbb{Q}_{p}^{*} \times \mathbb{R}^{*}$ and the inclusion is given by

$$
\mathbb{Q}_{p}^{*} \times \mathbb{R}^{*} \subset \mathbb{Q}_{p} \times \mathbb{R} .
$$

The role of the action of the group $\mathbb{K}^{*}$ by multiplication is now replaced by the action by multiplication by the group $U$ of elements of $\mathbb{K}^{*}=\mathbb{Q}^{*}$ which are units outside the above two places. This group is

$$
U=\mathbb{Z} / 2 \mathbb{Z} \times \mathbb{Z},
$$

where the $\mathbb{Z} / 2 \mathbb{Z}$ comes from \pm 1 and the cyclic group is $p^{\mathbb{Z}}$ generated by $p \in \mathbb{K}^{*}=$ $\mathbb{Q}^{*}$.

The action of $U$ of (10.12) on $\mathbb{R} \times \mathbb{Q}_{p}$ is given by the transformation

$$
S(x, y)=(p x, p y) .
$$

By comparison with the previous case of $\mathbb{K}=\mathbb{Q}(\sqrt{2})$, notice how in that case (cf. (10.2) ) the pair $\left(u, u^{-1}\right)$ was just the image of the element $3-2 \sqrt{2}$ under the diagonal embedding of $\mathbb{K}$ in $\mathbb{K}_{v_{1}} \times \mathbb{K}_{v_{2}}$.

In the present case, the role of the adeles class space $X_{\mathbb{K}}=\mathbb{A}_{\mathbb{K}} / \mathbb{K}^{*}$ is then played by its semi-local version

$$
X_{p, \infty}=\left(\mathbb{Q}_{p} \times \mathbb{R}\right) / U
$$

quotient of $\mathbb{Q}_{p} \times \mathbb{R}$ by the symmetry $(x, y) \rightarrow(-x,-y)$ and the transformation $S$. Both of these transformations preserve the function

$$
\tilde{f}: \mathbb{Q}_{p} \times \mathbb{R} \rightarrow \mathbb{R}_{+}, \quad \tilde{f}(x, y)=|x|_{p}|y| \in \mathbb{R}_{+},
$$

which descends to a function

$$
f: X_{p, \infty} \rightarrow \mathbb{R}_{+}
$$

Moreover, one has

$$
(x, y) \in \mathbb{Q}_{p}^{*} \times \mathbb{R}^{*} \subset \mathbb{Q}_{p} \times \mathbb{R} \Leftrightarrow f(x, y) \neq 0
$$

and the fiber of $f$ over any non zero $\varepsilon \in \mathbb{R}_{+}$is easily identified with $\mathbb{Z}_{p}^{*}$

$$
f^{-1}(\varepsilon) \sim \mathbb{Z}_{p}^{*}, \quad \forall \varepsilon \neq 0
$$

In fact, one can use the fundamental domain

$$
\mathbb{Z}_{p}^{*} \times \mathbb{R}_{+}^{*}
$$

for the action of $U$ on $\mathbb{Q}_{p}^{*} \times \mathbb{R}^{*}$ to obtain the required isomorphism. 


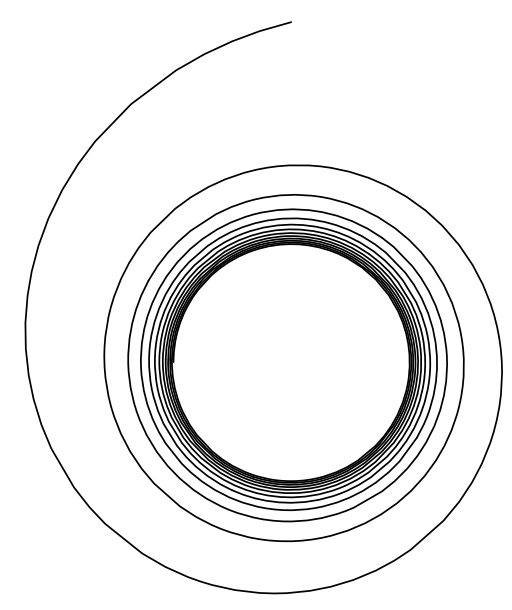

Figure 5. The limit cycle of a foliation.

The fiber $f^{-1}(0)$ of $f$ over the point $\varepsilon=0$ is no longer $\mathbb{Z}_{p}^{*}$ and once again it is singular. It is again described as the union of three pieces

$$
f^{-1}(0)=T_{p} \cup T_{\infty} \cup\{0\},
$$

which have, respectively, the following description.

- $T_{p}$ is the locus $x=0, y \neq 0$, which is identified with a torus $T_{p} \sim \mathbb{R}_{+}^{*} / p^{\mathbb{Z}}$, using the map $(x, y) \mapsto|y|$.

- $T_{\infty}$ is the locus $x \neq 0, y=0$, which gives the compact space $T_{\infty} \sim \mathbb{Z}_{p}^{*} / \pm 1$ obtained as quotient of $\mathbb{Q}_{p}^{*}$ by the action of $U$.

- The remaining piece is the point $x=0, y=0$.

The description of the topology of $f^{-1}(0)$ is similar to what happens in the case of $\mathbb{Q}(\sqrt{2})$ analyzed above.

What is not obvious in this case is how the totally disconnected fiber $f^{-1}(\varepsilon) \sim \mathbb{Z}_{p}^{*}$ can tie in with the torus $T_{p} \sim \mathbb{R}_{+}^{*} / p^{\mathbb{Z}}$ when $\varepsilon \rightarrow 0$.

To see what happens, we use the map

$$
X_{p, \infty} \ni(x, y) \mapsto g(x, y)=\text { class of }|y| \in \mathbb{R}_{+}^{*} / p^{\mathbb{Z}} .
$$

This is well defined on the open set $y \neq 0$. It is continuous and passes to the quotient. Thus, when a sequence $\left(x_{n}, y_{n}\right) \in X_{p, \infty}$ converges to a point $(0, y) \in T_{p}$, $y \neq 0$, one has $g(0, y)=\lim _{n} g\left(x_{n}, y_{n}\right)$.

The point then is simply that we have the relation

$$
g(x, y)=f(x, y) \in \mathbb{R}_{+}^{*} / p^{\mathbb{Z}} .
$$

In other words, $g\left(x_{n}, y_{n}\right)=\varepsilon_{n}$ with $\left(x_{n}, y_{n}\right)$ in the fiber $f^{-1}\left(\varepsilon_{n}\right)$ and the point of the singular fiber $T_{p}$ towards which $\left(x_{n}, y_{n}\right) \in X_{p, \infty}$ converges depends only on the value of $\varepsilon_{n}$ in $\mathbb{R}_{+}^{*} / p^{\mathbb{Z}}$. 
This phenomenon is reminiscent of the behavior of holonomy in the context of foliations, using a logarithmic scale $\mathbb{R}_{+}^{*} / p^{\mathbb{Z}} \sim \mathbb{R} /(\mathbb{Z} \log p)$. It corresponds to what happens in the limit cycle of the foliation associated to a flow as depicted in Figure 5 ,

As we argued in [11] (see also 93.2 and 97.1 here above), the role of Frobenius in characteristic zero is played by the one parameter group $\operatorname{Fr}(t)$ with $t \in \mathbb{R}$ which corresponds to the action of $\mathbb{R}$ on the adele class space $X_{\mathbb{Q}}=\mathbb{A}_{\mathbb{Q}} / \mathbb{Q}^{*}$ given in the above logarithmic scale, namely

$$
\operatorname{Fr}(t)(a)=e^{t} a, \quad \forall a \in X_{\mathbb{Q}} .
$$

Its orbit over $p \in \Sigma_{\mathbb{Q}}$ is of length $\log p$ and it corresponds, in the simplified picture of $X_{p, \infty}$, to the component $T_{p}$ of the singular fiber $f^{-1}(0)$.

10.3. Singularities of maps. The simple examples described above illustrate how one can use the function $f(x)=|x|$ in general, and see the place where it vanishes as the complement of $C_{\mathbb{K}}$ in the adeles class space $X_{\mathbb{K}}$. This provides a way of thinking of the inclusion of $C_{\mathbb{K}}$ in $X_{\mathbb{K}}$ in terms of the notions of "singular fiber" and "generic fiber" as seen in the examples above. The generic fiber appears to be typically identified with $C_{\mathbb{K}, 1}$, with the union of the generic fibers giving $C_{\mathbb{K}}$ as it should. This suggests the possibility of adapting to our noncommutative geometry context some aspects of the well developed theory of nearby and vanishing cycles. A brief dictionary summarizing this analogy is given here below.

\begin{tabular}{|c|c|}
\hline Total space & $f(x)=|x|$ \\
\hline The map $f$ & $X_{\mathbb{K}} \backslash C_{\mathbb{K}}=f^{-1}(0)$ \\
\hline Singular fiber & $C_{\mathbb{K}}=f^{-1}\left(\{0\}^{c}\right)$ \\
\hline Union of generic fibers & \\
\hline
\end{tabular}

\section{REFERENCES}

[1] Y. André, Une introduction aux motifs, Panoramas et Synthèses, Vol.17, Société mathématique de France, 2005.

[2] M.F. Atiyah, R. Bott, A Lefschetz fixed point formula for elliptic complexes: I, Annals of Math. Vol. 86 (1967) 374-407.

[3] S. Bloch, H. Esnault, D. Kreimer, On motives associated to graph polynomials, Commun. Math. Phys. Vol. 267 (2006) 181-225.

[4] E. Bombieri, Problems of the Millenium: The Riemann Hypothesis, Clay mathematical Institute (2000). 
[5] J.B. Bost, A. Connes, Hecke algebras, type III factors and phase transitions with spontaneous symmetry breaking in number theory. Selecta Math. (N.S.) 1 (1995), no. 3, 411-457.

[6] A. Connes, Une classification des facteurs de type III. Ann. Sci. École Norm. Sup. (4) 6 (1973), 133-252.

[7] A. Connes, Cohomologie cyclique et foncteurs Ext ${ }^{n}$. C. R. Acad. Sci. Paris Sér. I Math. 296 (1983), no. 23, 953-958.

[8] A. Connes, Noncommutative differential geometry. Inst. Hautes Études Sci. Publ. Math. No. 62 (1985), 257-360.

[9] A. Connes, Noncommutative geometry. Academic Press, 1994.

[10] A.Connes Trace formula in noncommutative geometry and the zeros of the Riemann zeta function. Selecta Math. (N.S.) 5 (1999), no. 1, 29-106.

[11] A. Connes, C. Consani, M. Marcolli, Noncommutative geometry and motives: the thermodynamics of endomotives, preprint math.QA/0512138.

[12] A. Connes, M. Marcolli, From physics to number theory via noncommutative geometry. Part I. Quantum statitical mechanics of $\mathbb{Q}$-lattices, preprint math.NT/0404128.

[13] A. Connes, M. Marcolli, Noncommutative geometry from quantum fields to motives (tentative title), book in preparation.

[14] A. Connes, M. Marcolli, N. Ramachandran, KMS states and complex multiplication, Selecta Math. (New Ser.) Vol.11 (2005), no. 3-4, 325-347.

[15] A. Connes, M. Marcolli, N. Ramachandran, KMS states and complex multiplication, II, in "Operator Algebras - The Abel Symposium 2004", pp.15-60, Springer Verlag, 2006.

[16] A. Connes, G. Skandalis, The longitudinal index theorem for foliations, Publ. RIMS Kyoto Univ. 20 (1984) 1139-1183.

[17] A. Connes, M. Takesaki, The flow of weights on factors of type III. Tohoku Math. J., 29, (1977) 473-575.

[18] C. Consani, Double complexes and Euler L-factors. Compositio Math. 111 (1998), no. 3, 323-358.

[19] C. Consani, M. Marcolli, Archimedean cohomology revisited, in "Noncommutative Geometry and Number Theory" pp.109-140. Vieweg Verlag, 2006.

[20] C. Consani, M. Marcolli, Quantum statistical mechanics over function fields, math.QA/0607363, to appear in Journal of Number Theory.

[21] P. Deligne, Théorie de Hodge III, Publ. Math. IHES Vol. 44 (1974) 5-78.

[22] A. Goncharov, Yu. Manin Multiple zeta motives and moduli spaces $\bar{M}_{0, n}$, Compos. Math. Vol. 140 no. 1 (2004) 1-14.

[23] V. Guillemin, S. Sternberg, Geometric asymptotics, Math. Surveys Vol. 14, American Mathematical Society, 1977.

[24] V. Guillemin, Lectures on spectral theory of elliptic operators, Duke Math. J., Vol. 44, 3 (1977), 485-517.

[25] E. Ha, F. Paugam, Bost-Connes-Marcolli systems for Shimura varieties. I. Definitions and formal analytic properties, IMRP Int. Math. Res. Pap. 2005, no. 5, 237-286.

[26] B. Jacob, Bost-Connes type systems for function fields, math.OA/0602554, to appear in Journal of Noncommutative Geometry.

[27] Yu.I. Manin, Correspondences, motifs and monoidal transformations, Mat. Sb. (N.S.) 77 (119) 1968, 475-507.

[28] Yu.I. Manin, Lectures on zeta functions and motives (according to Deninger and Kurokawa). Columbia University Number Theory Seminar (New York, 1992). Astérisque No. 228 (1995), 4, 121-163.

[29] Yu.I. Manin, Real Multiplication and noncommutative geometry (ein Alterstraum), in "The legacy of Niels Henrik Abel", pp.685-727, Springer, Berlin, 2004.

[30] Yu.I. Manin, Von Zahlen und Figuren, preprint arXiv math.AG/0201005.

[31] M. Marcolli, Arithmetic noncommutative geometry, University Lectures Series, Vol.36, American Mathematical Society, 2005. 
[32] R. Meyer, On a representation of the idele class group related to primes and zeros of $L$ functions. Duke Math. J. 127 (2005), no. 3, 519-595.

[33] B. Riemann, Mathematical Works, Dover, New York, 1953.

[34] H.P.F. Swinnerton-Dyer, Applications of Algebraic Geometry to Number Theory, Proceedings Symposia in Pure Math. Vol. XX (1969) 21-26.

[35] M. Takesaki, Tomita's theory of modular Hilbert algebras and its applications. Lecture Notes in Math., 28, Springer, 1970.

[36] M. Takesaki, Duality for crossed products and the structure of von Neumann algebras of type III, Acta Math. (131) (1973), 249-310.

[37] A. Weil, Letter to Artin, Collected Papers, Vol.I (1980) 280-298.

[38] A. Weil, On the Riemann hypothesis in function-fields, Proc. Nat. Acad. Sci. U.S.A. Vol.27 (1941) 345-347.

[39] A. Weil, Sur la théorie du corps de classes, J. Math. Soc. Japan, Vol.3 (1951) 1-35.

[40] A. Weil, Fonction zeta et distributions, Séminaire Bourbaki, Vol. 9 (1966), Exp. No. 312, $523-531$.

[41] A. Weil, Sur les formules explicites de la théorie des nombres premiers, Oeuvres compltes, Vol. 2, 48-62.

[42] A. Weil, Sur les formules explicites de la théorie des nombres, Izv. Mat. Nauk., (Ser. Mat.) Vol.36 (1972) 3-18.

[43] A. Weil, Basic Number Theory, Reprint of the second (1973) edition. Classics in Mathematics. Springer-Verlag, 1995.

A. Connes: Collège de France, 3, Rue d'Ulm, Paris, F-75005 France

E-mail address: alain@connes.org

C. Consani: Mathematics Department, Johns Hopkins University, Baltimore, MD 21218 USA

E-mail address: kc@math.jhu.edu

M. Marcolli: Max-Planck Institut für Mathematik, Vivatsgasse 7, Bonn, D-53111 GERMANY

E-mail address: marcolli@mpim-bonn.mpg.de 